\title{
Pre-clinical Anti-Addictive and Side- effect profiles of Novel Kappa-opioid Agonists
}

David Reid Young

2015

A thesis submitted to Victoria University of Wellington in fulfilment of the requirements for the degree of Master's of Biomedical Science 





\section{Abstract}

Background: Drug addiction is a chronic, relapsing disorder with great socioeconomic and morbidity costs. An estimated 27 million people worldwide suffer from drug dependence, with over 180,000 drug abuse-related deaths reported annually (UNODC, 2015). Currently, there are no FDA-approved pharmacotherapies for psychostimulant addiction, limiting the efficacy of treatment for cocaine and amphetamine abuse. Kappa-opioid receptor (KOPr) agonists can act as inhibitors of reward, and have been investigated in pre-clinical models of drug abuse for potential anti-addictive properties, but display undesirable side-effects such as dysphoria and sedation. A naturally-occurring KOPr agonist, Salvinorin A (SalA), has been explored as a lead for new KOPr-based antiaddictive medications. SalA is a short-acting but potent non-nitrogenous KOPr agonist with known anti-cocaine effects, and chemical alterations to this structure have produced novel agonists with comparable or greater potency at the KOPr. This thesis compares two novel SalA analogues, 16-ethynyl Salvinorin A (Ethy-SalA) and 16-methyl Salvinorin A (MeSalA), in pre-clinical models of addiction and side-effect tests.

Methods: Sprague-Dawley rats were used to model the behavioural effects of acute KOPr treatment upon cocaine self-administration and drug-seeking behaviour, natural rewardseeking, cocaine-induced and spontaneous locomotion, and pro-depressive forced-swim testing. Transiently co-transfected HEK-293 cells were used to model the influence of KOPr activation upon dopamine transporter (DAT) function in an in vitro model of dopamine uptake, using confocal microscopy to detect internalisation of the fluorescent DAT substrate ASP+.

Results: Acute pre-treatments of Ethy-SalA significantly attenuated cocaine-reinstatement of drug-seeking behaviour (at 0.1 and $0.3 \mathrm{mg} / \mathrm{kg}$ ) and progressive ratio (PR) selfadministration of cocaine (at $2.0 \mathrm{mg} / \mathrm{kg}$ ). The less potent agonist Me-SalA did not attenuate cocaine-reinstatement or PR self-administration at the doses tested $(0.3-2.0 \mathrm{mg} / \mathrm{kg})$. 
Despite apparent anti-cocaine effects, Ethy-SalA $(0.3 \mathrm{mg} / \mathrm{kg})$ was not found to effectively reduce cocaine-induced locomotor hyperactivity or sensitisation in rats. Side-effect screens were carried out on the novel compounds using the doses tested in cocaineprimed reinstatement. Ethy-SalA $(0.3 \mathrm{mg} / \mathrm{kg})$ and Me-SalA $(1.0 \mathrm{mg} / \mathrm{kg})$ did not significantly affect spontaneous locomotor behaviour $0.3 \mathrm{mg} / \mathrm{kg}$, or reduce self-administration of the natural reward sucrose at a dose of $0.3 \mathrm{mg} / \mathrm{kg}$ in rats. Depression-like effects caused by acute Ethy-SalA treatment $(0.3 \mathrm{mg} / \mathrm{kg})$ were also not detected in the Forced Swim Test. Treatment with Ethy-SalA $(10 \mu \mathrm{M})$ significantly increased uptake of the fluorescent ASP+ in co-transfected DAT/KOPr HEK-293 cells.

Conclusions: A single treatment of the novel KOPr agonist Ethy-SalA, but not the novel agonist Me-SalA, was found to attenuate drug-seeking behaviours in models of cocaine administration with greater potency than SalA, and without detectable sedative or depression-like effects at a dose of $0.3 \mathrm{mg} / \mathrm{kg}$. The cellular mechanism-of-action by which Ethy-SalA depresses cocaine reward is at least in part due to positive regulation of DAT, which would act to reduce extracellular dopamine within the brain. The lack of significant side-effects and the apparent improved potency of the compound support further exploration of Ethy-SalA as a lead for the development of an anti-addictive pharmacotherapy. 


\section{Acknowledgements}

None of the following would have happened without the support, encouragement, and occasional baked goods of many, many people, to whom I am all immensely grateful.

Firstly, and foremost, huge thanks go to my supervisor Dr. Bronwyn Kivell, whose advice, constant optimism and vision have driven this thesis. You have inspired me to push myself harder and to test the limits of my understanding and abilities. Thank you for trusting me with this project and encouraging me throughout these two years.

Thanks also to Dr. Peter Bosch, who took a very naïve Master's student and showed him how to be a scientist. Thank you for your incredible patience, and for all the guidance you gave me at the start of this project, without which I would have been very lost. To the soon-

to-be Dr. Amy Ewald, for her advice on everything from how to cope with becoming a science-hermit during postgrad, to the best way to dry a stressed, water-logged rat. Your tips, tricks, recipes and constant willingness to help have been absolutely invaluable.

To Fraser Putt, for all the help you gave whenever I was lost and bit panicked, and for doing some damn good surgeries for me. To Varun Venkatesh, for showing a clueless behavioural biologist how to do cell culture, and for all the great technique advice and the banter. To Sushila Pillai, for teaching me to use the vastly expensive, slightly terrifying and really fun piece of equipment that is the confocal microscope. Your trust, patience and expertise was wonderful to have.

To all of my lab mates: Kelly, Aimee, Adam, Diana, Susan, Caroline, Mohan, Jasleen, and Stephen. Thank you for the coffees, the baking, the science parties, and that little bit of crazy fun that you inject into lab life. You are all absolutely wonderful people, and I am honoured to have spent my two years with you. 
To all of the technician staff and facility managers, who fixed the things we broke, made the things we didn't have, and on one occasion took a door off its hinges so we could move something. Without you, we'd be sunk. So a huge thanks to Alan, Craig, Derek, and Shaun. Also, to the SBS staff, including Mary, Sandra, Lesley, Patricia, Mark and Paul, for all the many kindnesses you did for me.

A thank you, too, to the Awhina team, including Liz Richardson, Sonja Miller, Angela Thie and all the Awhina mentors and students. I've had the time of my life being part of the incredible whānau you've created. Haere rā.

To my family, for the constant support that you've given me throughout my tertiary education. Thank you for giving me somewhere to come to and relax when it got a bit tough. Thank you for your unwavering faith and love. And thank you for the care packages! To my partner Jen. You have been an absolute rock. Thank you from the bottom of my heart for putting up with the rants, the occasional tantrum, and the tears, and responding with unconditional baking and hugs. I'm ready to do just the same for you, anytime you ask.

And finally: to all the rats who I looked after and who gave their lives for science. 


\section{Table of contents}

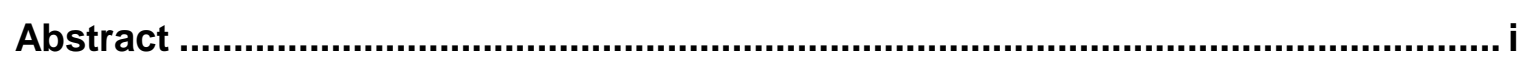

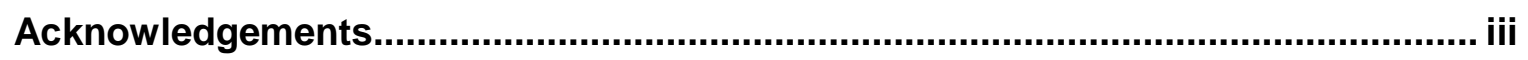

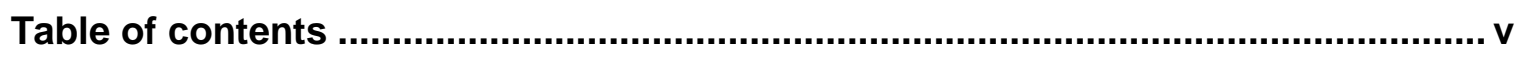

List of Figures .................................................................................................. ix

List of Tables ..........................................................................................................

Abbreviations ….............................................................................................. xii

Chapter 1 : Introduction ................................................................................... 1

1.1 Addiction: Definitions and Socioeconomic cost .............................................. 1

1.2 Neurophysiology of Drug Abuse and Addiction ........................................... 2

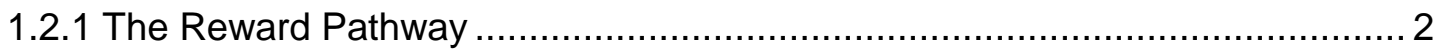

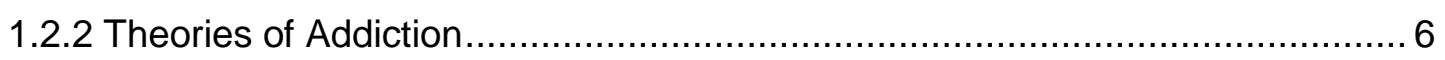

1.3 Behavioural Models of Addiction and Drug-seeking behaviour.......................... 8

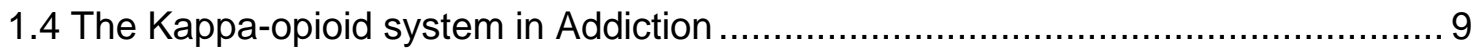

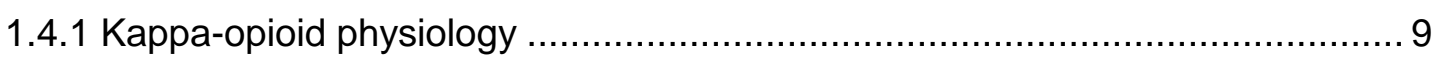

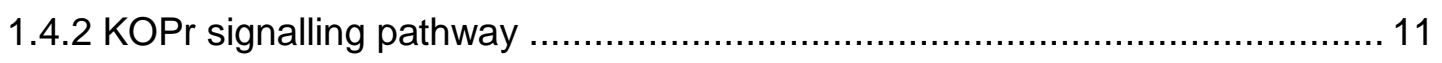

1.4.3 Kappa-opioids in addiction research.................................................... 15

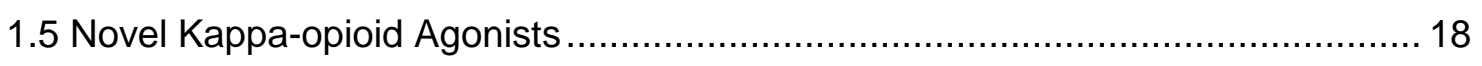

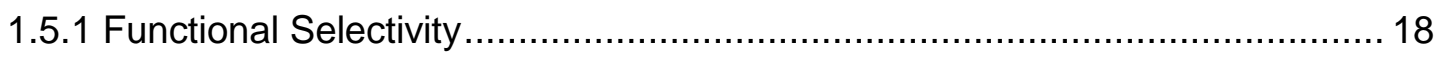

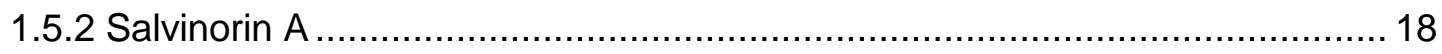

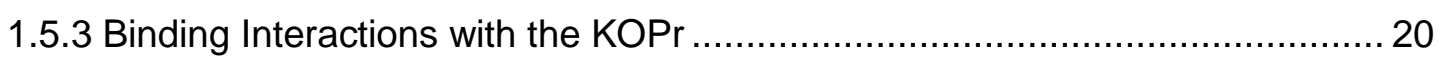

1.5.4 Synthesis of novel Salvinorin-based KOPr agonists ................................. 22

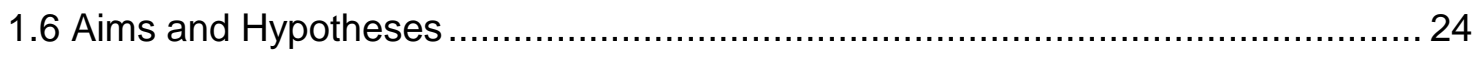

Chapter 2 : Behavioural anti-cocaine effects of novel KOPr agonists ..................... 25

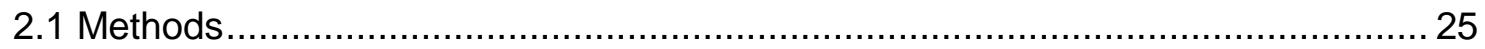

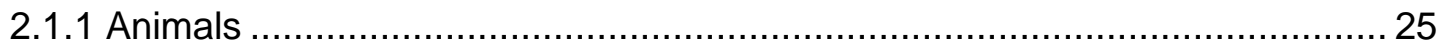

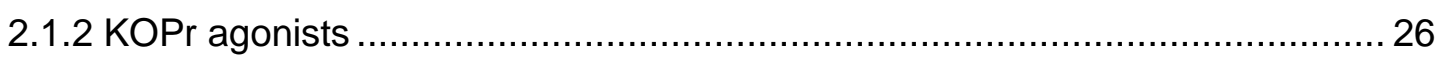

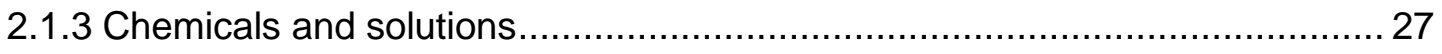


2.1.3 Self-administration intra-jugular surgerve ........................................... 27

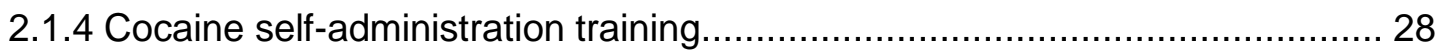

2.1.5 Cocaine-primed reinstatement f.......................................................... 29

2.1.6 Progressive-ratio cocaine self-administration ........................................ 30

2.1.7 Cocaine-induced locomotor hyperactivity ............................................ 33

2.1.8 Behavioural sensitisation to cocaine........................................................ 33

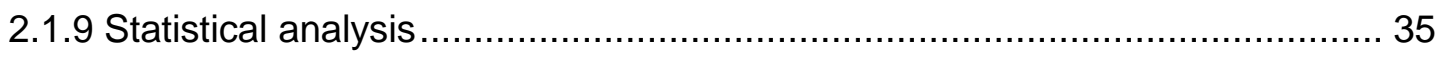

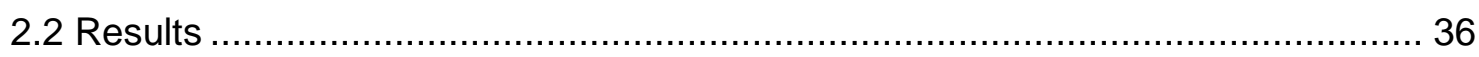

2.2.1 Operant training: cocaine self-administration.......................................... 36

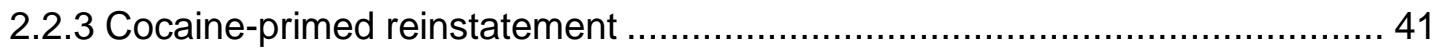

2.2.4 Progressive-ratio self-administration ................................................. 43

2.2.5 Cocaine hyperactivity with KOPr agonist pre-treatment............................ 45

2.2.6 Behavioural sensitisation to cocaine with acute KOPr treatment....................... 48

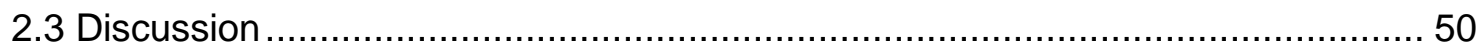

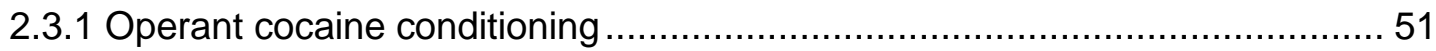

2.3.2 Models of drug relapse and reward motivation: cocaine-reinstatement and

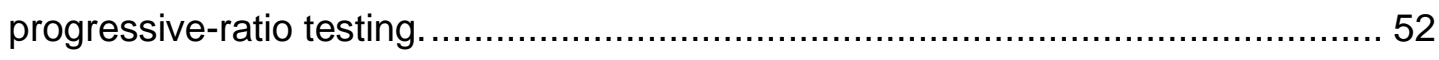

2.3.4 Models of cocaine locomotor effects: cocaine hyperactivity and behavioural

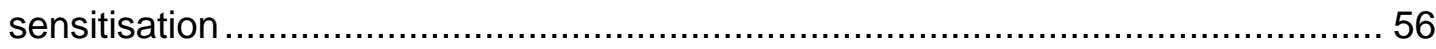

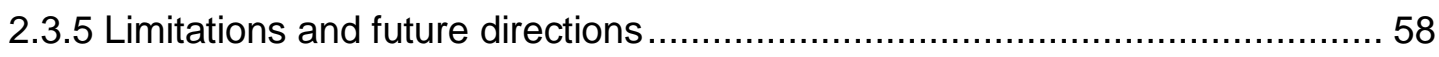

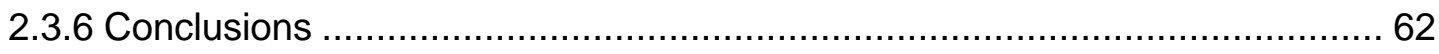

Chapter 3 : Behavioural side-effect testing of novel KOPr agonists.......................63

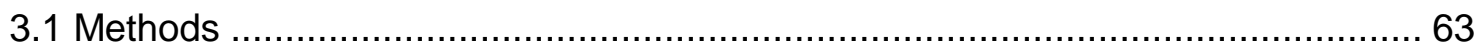

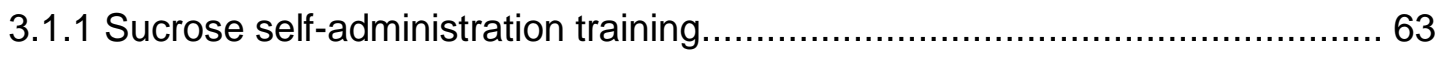

3.1.2 Sucrose administration with KOPr agonist pre-treatment .......................... 63

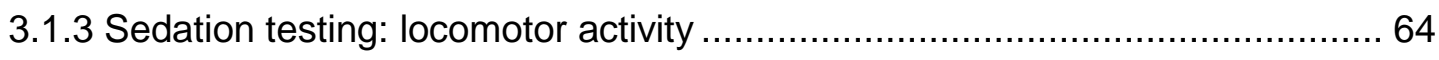

3.1.4 Pro-depressive screening: Forced Swim Test ...................................... 64

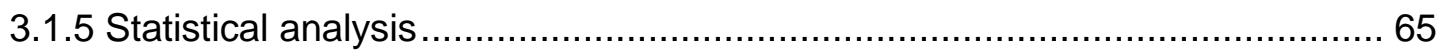

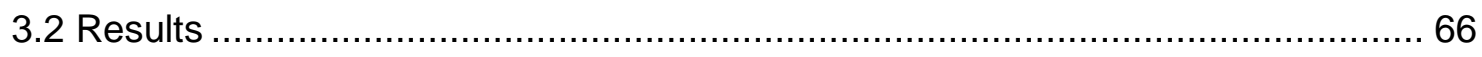

3.2.1 Effects of acute KOPr agonist treatment upon sucrose self-administration.... 66 
3.2.3 Locomotor activity profiles of Ethy-SalA and Me-SalA ..............................69

3.2.4 Forced Swim Test with acute KOPr agonist treatment............................... 72

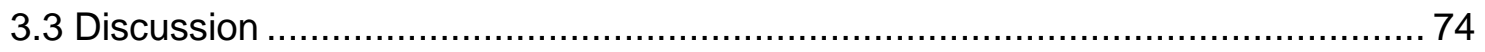

3.3.1 Modelling natural reward: sucrose self-administration ............................... 74

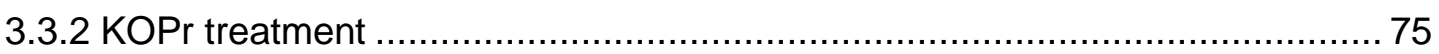

3.3.3 Sedative side-effects: locomotor activity testing ...................................... 76

3.3.4 Pro-depressive side-effects: the Forced Swim Test................................... 76

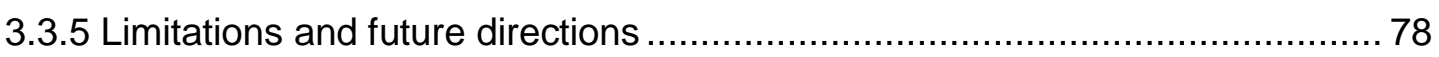

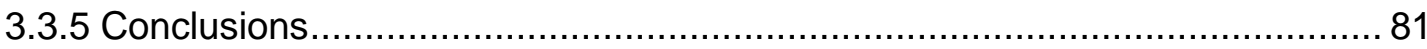

Chapter 4 : Cellular effects of Ethy-SalA: modelling dopamine re-uptake................ 82

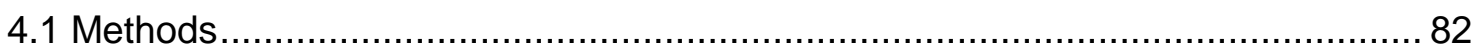

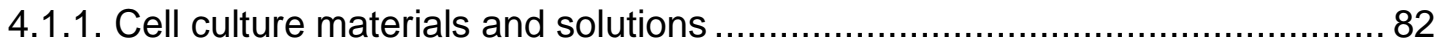

4.1.2 Cell culture- Human Embryonic Kidney cells (HEK-293).............................. 83

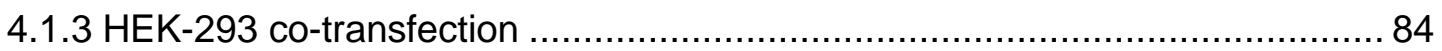

4.1.4 Confocal microscopy: trans-4-[4-(dimethylamino)styryl]-1-methylpyridinium

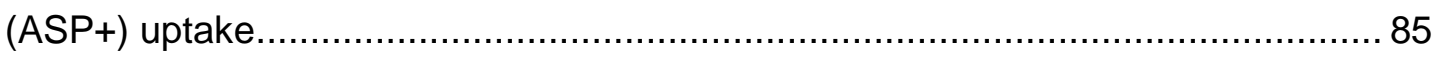

4.1.5 Confocal visualisation of myc-rKOPr expression ................................. 87

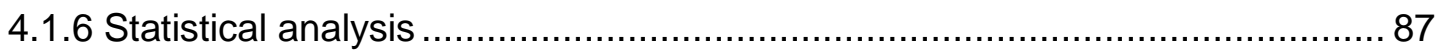

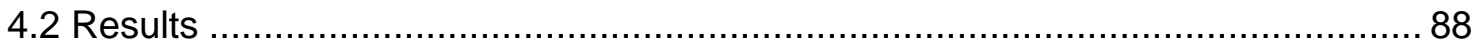

4.2.1 Co-expression of myc-rKOPr and YFP-hDAT in transfected HEK-293 cells .. 88

4.2.2 Characterisation of ASP+ uptake in DAT/KOPr co-transfected HEK 293 cells90

4.2.3 Changes to ASP+ uptake in DAT/KOPr co-transfected HEK-293 cells after

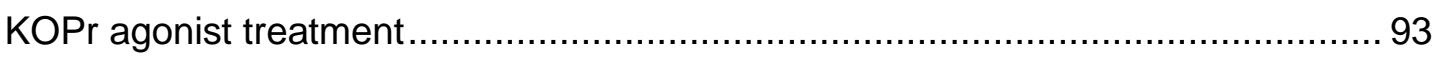

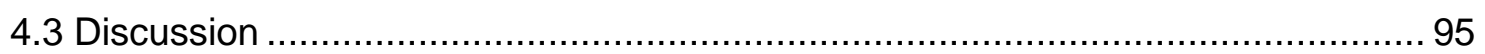

4.3.1 YFP-hDAT and myc-rKOPr co-expression in HEK293 cells......................... 95

4.3.2 Interaction of KOPr and DAT to enhance ASP+ uptake ............................... 95

4.3.3 Limitations and future directions ........................................................... 97

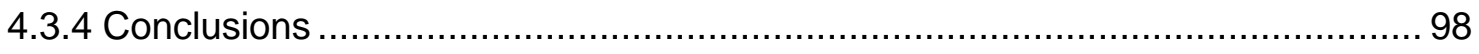

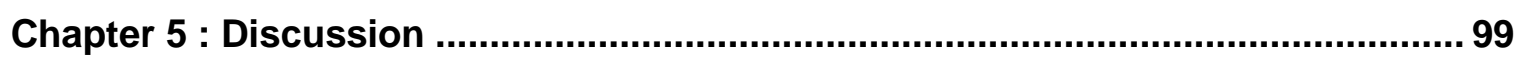

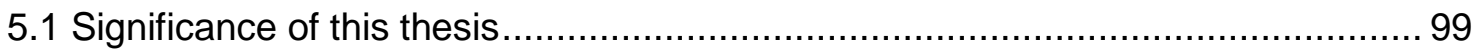


5.2 Summary of key findings

5.2.1 Acute treatment with Ethy-SalA attenuates responding in cocaine selfadministration behavioural models

5.2.2 Acute Ethy-SalA treatment does not affect cocaine-induced hyperactivity or sensitisation in rats 100

5.2.3 Low doses of Ethy-SalA and Me-SalA do not show sedative or pro-depressive side-effects, or attenuate natural reward in rats 100

5.2.4 Ethy-SalA treatment enhances DAT uptake of ASP+ in an in vitro cellular model. 101

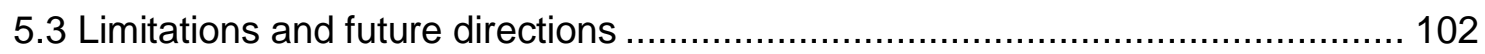

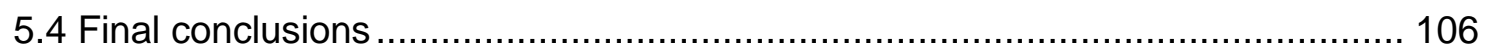

Chapter 6 : Supplementary information .................................................................... 107

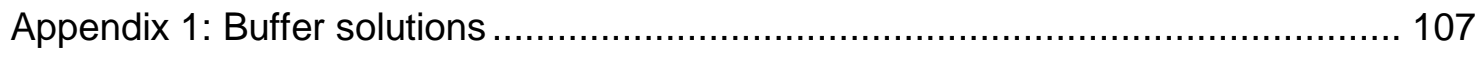

Appendix 2: In silico modelling of novel KOPr agonists ...................................... 108

Appendix 3.1: Catheter preparation for surgery ............................................... 110

Appendix 3.2: Intra-jugular catheter surgery .............................................. 111

Appendix 3.3: Post-operative care ..................................................................... 113

Appendix 4: Latin square treatment designs (self-admin) .................................. 114

Appendix 5: Cocaine hyperactivity with SalA pre-treatment ............................... 116

Appendix 6: Rat/Human KOPr homology....................................................... 117

Appendix 7: ASP+ accumulation and YFP-hDAT expression............................... 118

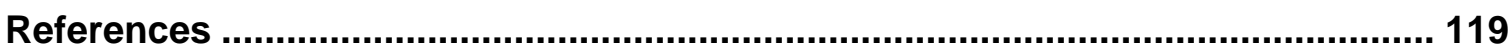




\section{List of Figures}

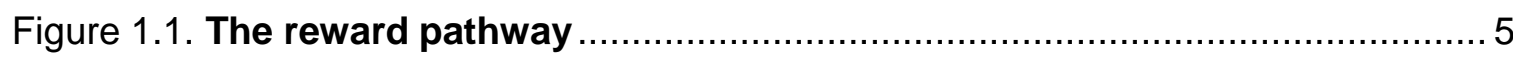

Figure 1.2. An illustration of the three-stage addictive cycle ............................... 7

Figure 1.3. Summary of KOPr signalling pathways and effects ........................... 13

Figure 1.4. KOPr and DAT co-expression in the brain ...................................... 14

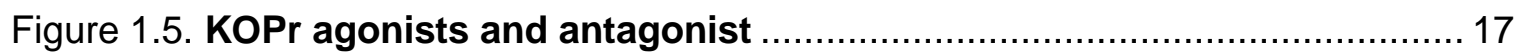

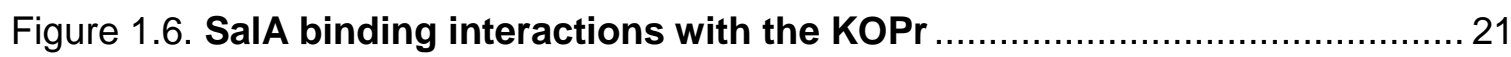

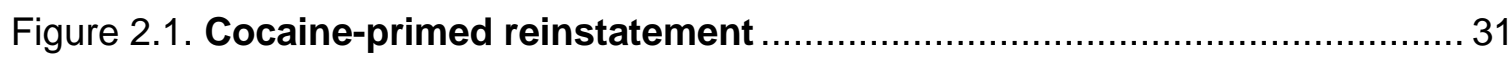

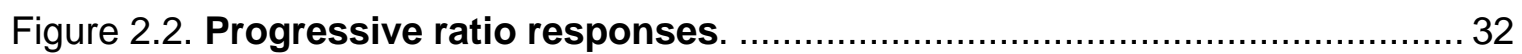

Figure 2.3. Treatment schedule for cocaine sensitisation ................................... 34

Figure 2.4. Acquisition of cocaine self-administration .......................................... 38

Figure 2.5. Baseline cocaine self-administration responses ............................... 39

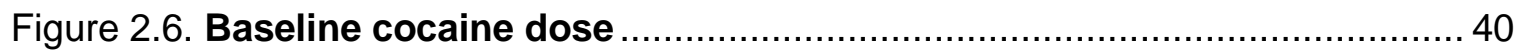

Figure 2.7. Cocaine-primed reinstatement of self-administration behaviour with

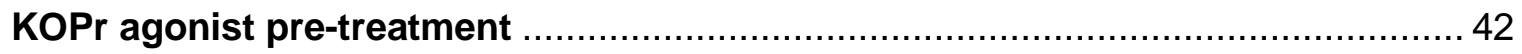

Figure 2.8. Progressive ratio cocaine self-administration with KOPr agonist pre-

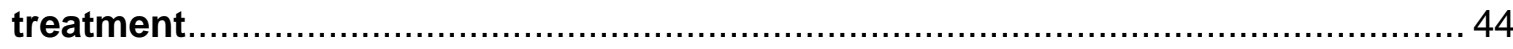

Figure 2.9. Measures of Cocaine hyperactivity with KOPr agonist pre-treatment... 46

Figure 2.10. Time course analysis of cocaine hyperactivity with KOPr agonist pre-

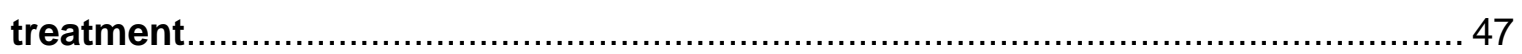

Figure 2.11. Cocaine sensitisation testing with KOPr agonist pre-treatment ..........50

Figure 3.1. Effects of acute KOPr agonist treatment upon sucrose self-

administration

Figure 3.2. Baseline sucrose self-administration responses................................. 68

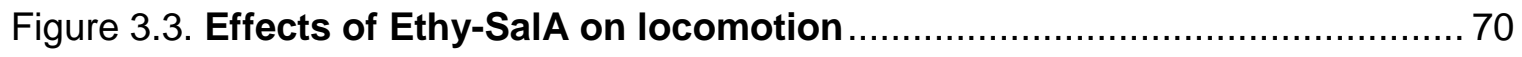

Figure 3.4. Effects of Me-SalA on locomotion.............................................. 71

Figure 3.5. Forced Swim Test with acute KOPr agonist treatment ......................... 73 
Figure 4.1. Co-expression of myc-rKOPr and YFP-hDAT in transfected HEK-293

cells

Figure 4.2. Time course images of ASP+ uptake in co-transfected DAT/KOPr HEK293 cells

Figure 4.3. Linear ASP+ uptake in DAT/KOPr co-tranfected HEK-293 cells 92

Figure 4.4. Changes to ASP+ uptake in YFP-hDAT and myc-rKOPr co-transfected HEK-293 cells after KOPr agonist treatment .94

Figure 6.1. Computer-generated modelling of KOPr compounds 109

Figure 6.2. Intra-jugular catheter 110

Figure 6.3. Catheter placement 112

Figure 6.4. SalA cocaine hyperactivity 116

Figure 6.5. Correlation of YFP-hDAT expression and cellular ASP+ accumulation in untreated transfected HEK-293 cells 118 


\section{List of Tables}

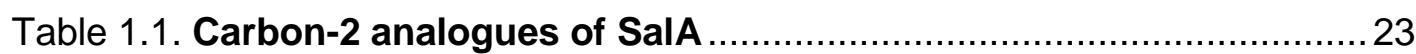

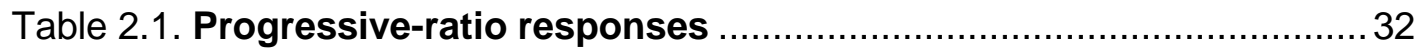

Table 2.2. Carbon-16 analogues of SalA ..................................................... 54

Table 6.1. Cocaine reinstatement treatments. ........................................ 114

Table 6.2. Progressive-ratio treatments ................................................ 115

Table 6.3. Sucrose self-administration treatments................................. 115 


\section{Abbreviations}

5-HT

$\beta$-THP SalB

Br-SalA

BSA

cAMP

CREB

$\mathrm{CHO}$

CNS

CPA

CPP

$\mathbf{D}_{1}$

$\mathrm{D}_{2}$

DA

DAT

EOM-SalB

Ethy-SalA

FST

GPCR

i.v.

$\mathrm{KOPr}$

LSD

MAPK
Serotonin

$\beta$-tetrahydropyran Salvinorin B

16-bromo Salvinorin A

Bovine serum albumin

cyclic Adenosine Monophosphates

cAMP response element-binding protein

Chinese Hamster Ovary

Central Nervous System

Conditioned Place Aversion

Conditioned Place Preference

Dopamine 1 receptor

Dopamine 2 receptor

Dopamine

Dopamine transporter

2-ethoxymethyl Salvinorin B

16-ethynyl Salvinorin A

Forced Swim Test

G-protein coupled receptor

Intravenous

Kappa-opioid peptide receptor

Lysergic acid diethylamide

Mitogen-Activated Protein Kinase 
MDMA

Me-SalA

MOM-SalB

MOR

NAc

NorBNI

PBS

pFC

SalA

SalB

SERT

s.c.

$\mathrm{U}-50,488$

U-69,593

VTA

YFP 3,4-methylenedioxy- $N$-methylamphetamine

16-methyl Salvinorin A

2-methoxymethyl Salvinorin B

Mu-opioid receptor

Nucleus accumbens

Norbinaltorphimine

Phosphate-buffered saline

Pre-frontal cortex

Salvinorin A

Salvinorin B

Serotonin transporter

subcutaneous

trans-( \pm )-3,4-Dichloro-N-methyl-N-[2-(1-

pyrrolidinyl)cyclohexyl]benzeneacetamide

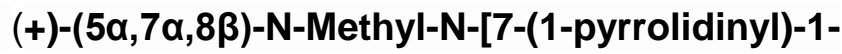
oxaspiro[4.5]dec-8-yl] benzeneacetamide

Ventral tegmental area

Yellow fluorescent protein 



\section{Chapter 1 : Introduction}

\subsection{Addiction: Definitions and Socioeconomic cost}

Addiction is one of the most debilitating outcomes associated with drug abuse, and is a significant barrier to the cessation of drug use. The addictive condition is defined as a chronic, relapsing disorder associated with long-term substance abuse (Rinaldi et al., 1988; WHO., 2010). Addiction is not unanimous amongst drug users, but population studies in the USA and Australia indicate that around one in five people who regularly use illicit drugs will go on to develop drug dependence (Glantz et al., 2009; Grant, 1996; Hall et al., 1999).

Globally, drug abuse accounts for a substantial economic burden through early morbidity, crime, and treatment costs (Andlin-Sobocki \& Rehm, 2005; Cartwright, 2008; Harwood et al., 1999; Wickizer, 2013). Global mortality as a result of drug abuse is estimated at 187,100 deaths per year, and only one in six drug abusers has access to treatment or interventions (UNODC, 2015). Psychostimulants, such as cocaine, amphetamine-like stimulants, and opioids such as heroin are among the most heavily abused drugs (UNODC, 2015). In New Zealand, approximately $17 \%$ of people over the age of 16 years admit to recreational use of illicit drugs (Mason et al., 2010), with $83 \%$ of regular opioid users, $55 \%$ of regular methamphetamine users, and $10 \%$ of regular 3,4 -methylenedioxy$\mathrm{N}$-methylamphetamine (MDMA) or 'ecstasy' users being classified as dependent upon their drug of abuse (Wilkins et al., 2008). Drug use in New Zealand is also strongly associated with criminal activity; in a survey of the major cities Auckland, Wellington and Christchurch across 2006 and 2007, 16\% of frequent illicit drug users were reported to have committed theft within the past month, $6 \%$ to have committed violent crime, and $38 \%$ to be dealing illegal drugs themselves (Wilkins et al., 2008). The cumulative costs of drug and alcohol abuse in New Zealand have been estimated at $\$ 6.525$ billion NZD (BERL- 
Economics., 2009). In the United States of America, a similar estimate based upon 2007 data suggested total costs of approximately $\$ 193$ billion USD (NDIC, 2011).

Heroin, cocaine, and amphetamines are some of the most heavily abused drugs worldwide, and contribute to the majority of drug-abuse cases (UNODC, 2015). Demand for cocaine-related treatment is highest in North and South America (UNODC, 2015; WHO., 2010), and though there are indications that cocaine availability and abuse in the U.S. and Europe is decreasing in the face of increased heroin, methamphetamine and prescription opioid abuse, cocaine still represents a significant global problem in terms of abuse and addiction (NDIC, 2011; UNODC, 2015). In New Zealand cocaine is a comparatively much rarer drug of abuse, though a 2005-2007 survey indicated that cocaine availability was trending towards an increase (Wilkins et al., 2008). Successful rehabilitation treatment for drug addiction has the potential for significant economic benefits to both society and the individual, by reducing costs associated with crime and treatment (Flynn et al., 1999; Prendergast et al., 2002). Effective treatment for addiction is therefore a highly desirable outcome, and though pharmacology-based therapies exist for alcohol and opiate dependence, there are currently no treatments for psychostimulants approved by the Food and Drug Administration (FDA).

\subsection{Neurophysiology of Drug Abuse and Addiction}

\subsubsection{The Reward Pathway}

Drug abuse is intrinsically tied to the mesolimbic and mesocortical dopaminergic pathways of the brain, often referred to collectively as the 'reward pathway'. The three key areas of the brain involved in the generation of reward are the ventral tegmental area (VTA), the nucleus accumbens (NAc) and the pre-frontal cortex (pFC) (Phillips \& Fibiger, 1978). The major biochemical basis for the sensation of reward is the neurotransmitter dopamine (DA) 
(Carlsson et al., 1957; Simon et al., 1979), which is synthesised within the VTA by dopaminergic neurons with extensions to the NAc and pFC (see Fig.1.1), forming the respective mesolimbic and mesocortical neural pathways (Swanson, 1982; Wood \& Rao, 1991). Stimulation of the VTA results in release of DA at synaptic terminals in the NAc and pFC, evoking a pleasurable or euphoric feeling in response to behavioural stimuli such as the ingestion of food and drink (Yoshida et al., 1992) and sexual activity (Pfaus et al., 1990). The actions of DA upon post-synaptic target cells in the NAc and pFC are mediated by a family of G-protein coupled receptors, generally ordered into the major groupings of $D_{1}$-like and $D_{2}$-like receptors according to their respective stimulatory or inhibitory intracellular interactions with adenylyl cyclase (Kebabian, 1978; Self et al., 1996). In studies of the interaction of cocaine and amphetamines with the reward pathway, it has been identified that $D_{1}$ receptor-signalling enhances drug reward (Graham et al., 2007), while striatopallidal $D_{2}$ receptor-signalling acts to inhibit pro-reward processes (Durieux et al., 2009), in part by enhancing the activity of the DA transporter (DAT) and increasing DA uptake (Bolan et al., 2007).

All known drugs of abuse cause hyperactivity of the reward pathway, by increasing extracellular levels of DA (Di Chiara \& Imperato, 1988). The mechanisms by which drugs of abuse achieve this are many and varied; cocaine is one of the most widely studied, and is known to increase synaptic levels of DA by binding to and inhibiting the actions of the DAT, which normally acts to clear the synapse after DA release (Cass et al., 1992; Ritz et al., 1987). Inhibition of DAT results in a prolonged period of dopaminergic stimulation, which translates to the potent 'high' experienced by users of the drug (Volkow et al., 1997).

The abnormal stimulation of the reward pathway by drugs of abuse can induce significant neurophysiological changes in regular abusers. Chronic exposure to the drug cocaine is thought to induce a variety of neurophysiological changes, including alterations to $D_{1}$ and $D_{2}$ receptor expression and activity, with $D_{1}$ activation being favoured over D2 (Navarro et al., 2013; Park et al., 2013); and depletion of mesolimbic DA (Taylor \& Ho, 1977), 
potentially as a result of decreased expression of the vesicular monoamine transporter-2 (VMAT2), an important pre-synaptic regulator of DA storage (Little et al., 2003). Decreased VMAT2 is thought to stimulate a reciprocal down-regulation of DA storage vesicles, and thus an overall decreased pool of DA (Narendran et al., 2012). Positron emission tomography (PET) imaging studies of dopaminergic activity in human methamphetamine and cocaine abusers, using $D_{2}$ receptor radio-ligands, has indicated a loss of dopaminergic function in long-term abusers in comparison to non-abusers (Volkow et al., 2001; Volkow et al., 1993). Taken together, these studies suggest that long-term drug abuse can lead to DA imbalances and general dysregulation within the reward pathway. 

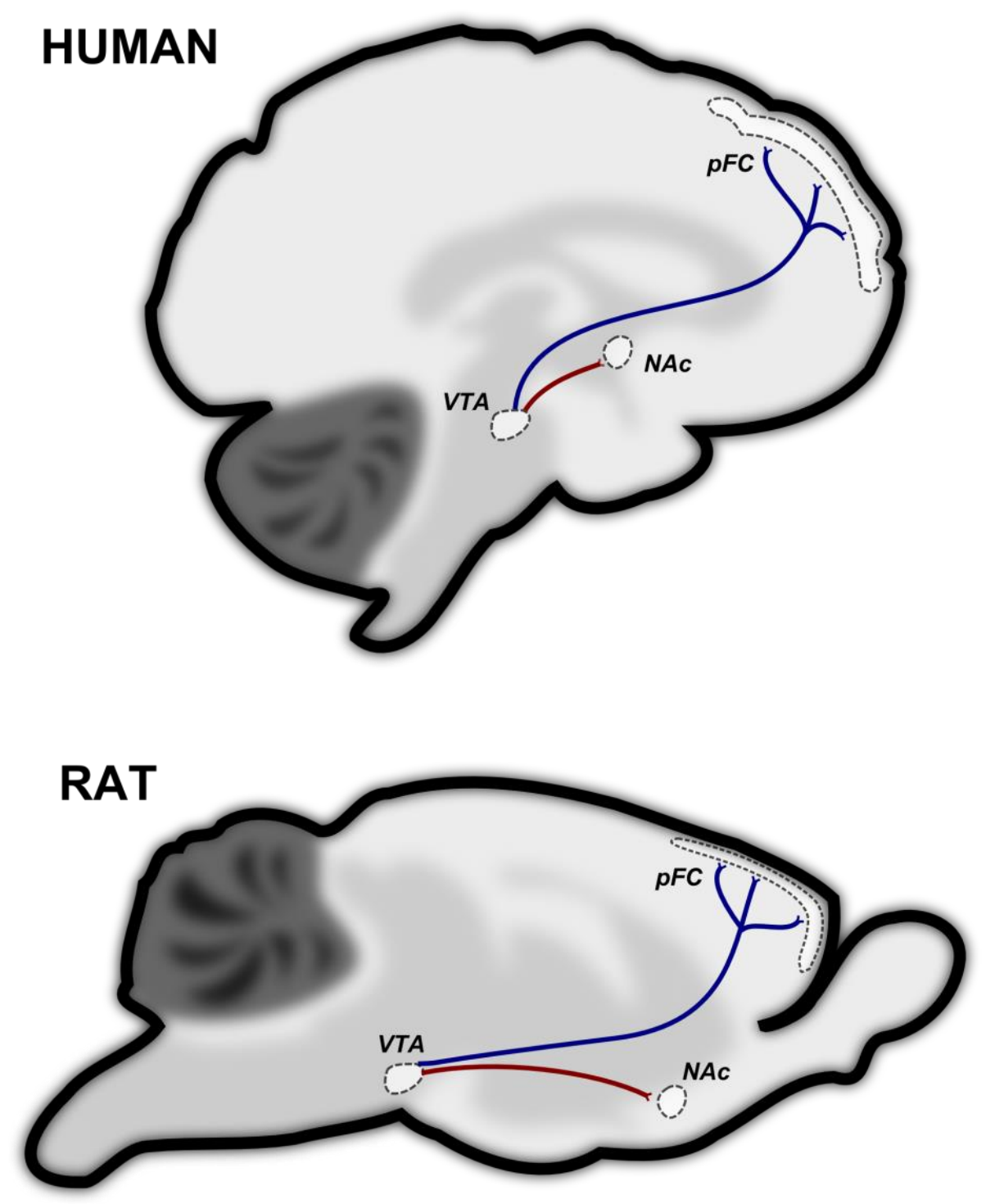

Figure 1.1. The reward pathway. A representation of the mesolimbic (dark red) and mesocortical (dark blue) dopaminergic projections of the VTA, which form the reward pathway in human and rat brains. The reward pathway and its components are well preserved between humans and rats, and the rat is a commonly-used animal model for human reward and drug-associated behaviours. VTA= ventral tegmental area; NAc= nucleus accumbens; $\mathrm{pFC}=$ pre-frontal cortex. 


\subsubsection{Theories of Addiction}

Long-term drug abusers are among the most likely to develop dependence or addiction upon their drug of choice. A repeating pattern of behaviour is formed, with periods of drug abuse and intoxication immediately followed by a dysphoric withdrawal state, characterised by an obsession or craving for more drug to alleviate negative withdrawal symptoms (see Fig. 1.2), which in turn leads to a relapse to drug taking (Koob \& Bloom, 1988; Koob \& Moal, 1997). Detoxified cocaine addicts, when exposed to the cocaine-like drug methylphenidate, show decreased DA activity in the striatal (NAc-containing) region, but increased activity in the thalamus, an area strongly associated with drug craving (Volkow et al., 1997). Two theories currently exist to explain the role of DA in the development of an addicted state; the first, the 'dopamine-depletion' hypothesis (Dackis \& Gold, 1985) posits that increased craving can be attributed to depleted stores as a result of chronic hyper-stimulation. The second theory, of 'incentive-sensitization', proposes that chronic abuse induces neurophysiological adaptations, causing the dopaminergic pathway to become highly sensitised to the drug of abuse (Robinson \& Berridge, 1993). In both models, the abused substance then becomes the only means to reduce the negative sensations of withdrawal, and induces strong cravings which can propel drug abusers into the addiction paradigm. 

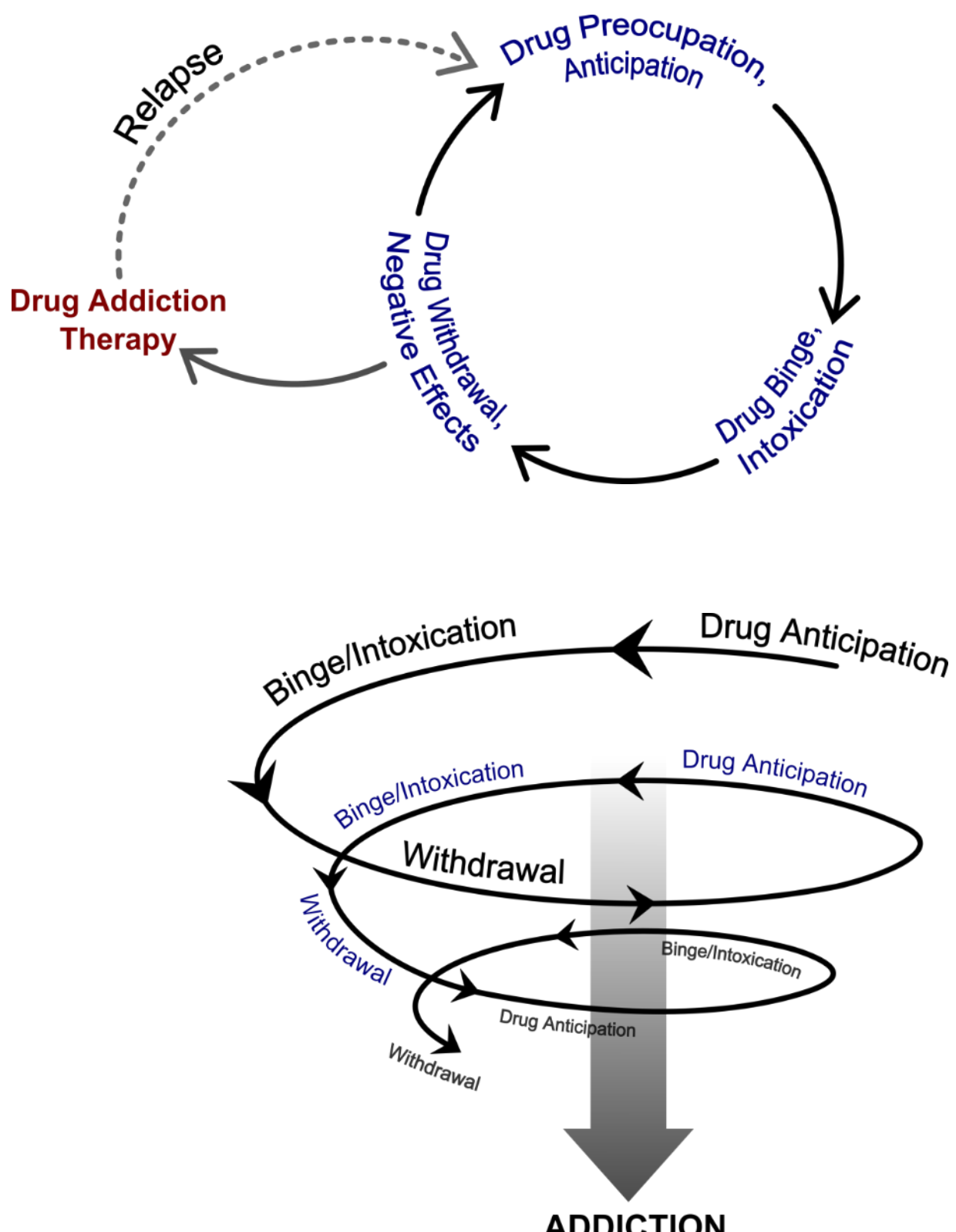

Figure 1.2. An illustration of the three-stage addictive cycle. This cycle is selfperpetuating, as preoccupation or 'craving' for the drug promotes drug intoxication, in turn inducing a negative withdrawal state which causes further drug craving to alleviate the negative effects of withdrawal. Addiction therapy attempts to break drug abusers out of the cycle at the withdrawal stage; if unsuccessful, those being treated will often relapse to drug abuse (top). The cycle can also be visualised as a descending spiral, where perpetuation of the cycle drives drug abusers further and further towards an addicted state (bottom). Modified from Koob and Le Moal (1997). 


\subsection{Behavioural Models of Addiction and Drug-seeking behaviour}

In order to better study the phenomenon of addiction, two animal models for human drugseeking behaviour are most commonly used: drug self-administration (in rodents or nonhuman primates) and conditioned place preference (in rodents) (CPP) (Balster, 1991; Tzschentke, 1998). In the self-administration procedure, first described by Weeks (1962), animals are conditioned to associate drug delivery with a physical action of a lever press, and subsequent measures of their responses can be used as a measure of drug-seeking behaviour (Panlilio \& Goldberg, 2007). Self-administration studies are most commonly performed in rats, with drug being delivered intravenously through a catheter inserted into the jugular vein and attached to a delivery mechanism which responds to lever presses performed by the rat within an operant box (de Wit \& Stewart, 1981; Weeks, 1962). The drug administered acts as a highly effective positive reinforcement for the required behaviour (Weeks, 1962), particularly in the case of cocaine: if allowed unlimited access, animals sensitised to cocaine will nearly continuously self-administer, even when placed on a reinforcement schedule which demands multiple responses from the animal in order to receive a single unit of drug (Griffiths et al., 1975).

Different types of self-administration regimens can be used to test the different effects of drugs of abuse. The drug-primed reinstatement model (de Wit \& Stewart, 1981) has been used as an approximation of human relapse to drug abuse (Bossert et al., 2013; Shaham \& Miczek, 2003), where abstinent individuals return to drug-seeking and drug-taking behaviour after acute exposure to the drug of abuse (de Wit, 1996) or a drug-associated environmental cue (Childress et al., 1993; O'Brien et al., 1992). The progressive-ratio model, where subjects are required to increase their responses in a roughly exponential pattern to continue to receive infusions (Depoortere et al., 1993; Hodos, 1961), measures the motivation of test subjects to continue seeking drug (Richardson \& Roberts, 1996). Progressive-ratio has been previously used to examine differences in response in animals 
previously sensitised to an abusive drug (Mendrek et al., 1998), comparisons of reinforcement between different drugs (Roberts, 1993), and the effect of a pre-treatment upon the reinforcing effects of a drug of abuse (McGregor et al., 1993). Changes to a 'break-point', or the point at which an animal will cease to attempt to self-administer the drug on offer, are used to gauge the subject's relative level of motivation (Stafford et al., 1998). As well as confirming the reinforcing effects of drugs of abuse, self-administration models in both rats and non-human primates have also been used to test potential antiaddictive pharmacotherapies by screening the ability of various compounds, including kappa-opioid receptor (KOPr) agonists to reduce drug-seeking behaviour (Cappendijk \& Dzoljic, 1993; Heidbreder \& Shippenberg, 1994; Schenk et al., 1999).

The CPP protocol tests 'preference' for a drug by causing the test subject to associate delivery of the drug with a particular environment (Bardo \& Bevins, 2000; Rossi \& Reid, 1976). Animals are also given a neutral or 'vehicle' treatment in a different environment.

On the test day the subject (usually mice or rats) is primed with an injection of the drug and then allowed to explore both 'drug' and 'vehicle' environments (Rossi \& Reid, 1976). If the animal chooses to spend more time in the environment in which they first received drug, they are said to display 'preference' for drug.

\subsection{The Kappa-opioid system in Addiction}

\subsubsection{Kappa-opioid physiology}

The kappa-opioid (KOP) system is an important regulator of mood within the central nervous system, and the endogenous KOP agonist dynorphin is thought to play an important role in mediating stress-associated dysphoria (Land et al., 2008). Dynorphin and its target, the G-protein coupled kappa-opioid receptor (KOPr), also have important antiaddictive regulatory functions by acting to oppose the dopaminergic effects and 
neurophysiological changes induced by long-term drug abuse (Mysels \& Sullivan, 2009; Spanagel et al., 1992). Synthetic KOPr agonists are being explored as an alternative target to DA receptor or DAT antagonism for anti-addiction pharmacotherapies (Prisinzano et al., 2005). Within the reward pathway, the KOP system acts to inhibit DA release in the NAc and pre-frontal cortex through negative regulation of dopaminergic neurons extending from the VTA (Margolis et al., 2006; Shippenberg et al., 2007), and also increases DA uptake by DAT (Thompson et al., 2000).

Long-term, the activity of dynorphin is also thought to encode the depressive and dysphoric components of drug withdrawal (Chartoff et al., 2012), and, together with the corticotrophin-releasing factor (CRF) activity, is strongly implicated as an important mediator of aversive stress responses (Bruchas et al., 2010). Exposure to drugs of abuse causes marked changes in KOP expression and activity, particularly in areas of the brain associated with reward. Increased dynorphin expression is observed in the striatal region of the brains of human cocaine addicts (Hurd \& Herkenham, 1993) and also within animal subjects given chronic cocaine treatment (Carlezon et al., 1998), suggesting that potentiation of the KOP system occurs as a result of long-term abuse. Successful stressinduced reinstatement to drug-seeking behaviour using acute KOPr agonist treatment has been generated in animal models of alcohol (Funk et al., 2014) and cocaine selfadministration (Redila \& Chavkin, 2008; Valdez et al., 2007) and can be blocked by KOPr antagonists (Beardsley et al., 2005), indicating that the dysphoric state caused by KOPr activation can act as a negative reinforcer of drug-seeking behaviour. Acute drug exposure can also influence KOP activity; a single injection of amphetamine has been shown to desensitise KOP receptors within the rat NAc, an effect which lasts for at least 5 days after amphetamine treatment (Xia et al., 2008).

Not all of the KOP-mediated effects in the brain appear to be aversive, however. A recent study using selective optogenetic activation of dynorphin-expressing cells of the ventral and dorsal regions of the NAc have indicated that while KOP activity within the ventral 
region of the NAc does produce an aversive response in place preference tests in mice, activation of dorsal KOP-expressing cells can induce a reward response (Al-Hasani et al., 2015). Similarly, other research has identified the presence of KOP-activated pro-reward "hot-spots" within a rostral region of the rat NAc shell (Castro \& Berridge, 2014). The contribution of the KOP system to behaviour is clearly much more complex than its traditionally assigned role as a mediator of aversive and dysphoric states of emotion.

\subsubsection{KOPr signalling pathway}

$\mathrm{KOPr}$ is a transmembrane G-protein coupled receptor (GPCR) widely expressed in the central and peripheral nervous systems; KOPr expression is particularly high in areas of the brain associated with reward, pain perception, and learning and memory (Simonin et al., 1995). KOPr activation generally has an inhibitory effect upon KOPr-expressing cells, which include nociceptive neurons in the periphery, and dopaminergic neurons in the central nervous system (CNS). According to one model (Bruchas \& Chavkin, 2010), agonist-binding to the KOPr activates Gai protein subunits, whose major downstream effect is the inhibition of cyclic-AMP synthesis by adenylyl cyclase (Taussig et al., 1993) and activation of the inflammatory and stress-associated c-Jun N-terminal Kinase (JNK) (Kam et al., 2004), while KOPr-mediated activation of Gyß protein subunits is thought to influence $\mathrm{K}^{+}$and $\mathrm{Ca}^{2+}$ channel activity, increasing membrane potentials and inhibiting action-potential transmission (Eriksson et al., 1993; Grudt \& Williams, 1993). The KOPr also activates the extracellular signal-regulated kinases 1 and $2(E R K 1 / 2)$ in two distinct stages, termed 'early' and 'late' phase activation Early phase activation appears to be Gy $\beta$ -mediated, while late-phase ERK1/2 activation occurs as a result of $\beta$-arrestin recruitment by the KOPr (McLennan et al., 2008) (see Fig. 1.3 for summary). The $\beta$-arrestin-dependant pathway includes activation of both ERK1/2 and p38 mitogen-activated protein kinase (MAPK), a combination which is thought to be responsible for many of the negative side- 
effects associated with classic KOPr agonists. The actions of p38 MAPK in particular have been strongly implicated in the development of aversion and negative stress-induced behaviours in animal models (Bruchas et al., 2011). It is also suspected that $\beta$-arrestin mediated signalling is responsible for human KOPr desensitisation, by stimulating receptor internalisation (Li et al., 1999). The downstream actions of $\beta$-arrestin recruitment therefore greatly hinder the therapeutic application of classical KOPr agonists, both as analgesic agents and as potential anti-addictive therapies.

KOP receptors have been shown to regulate DAT and serotonin (5-HT) transporter (SERT) function as part of an ERK1/2 dependant pathway (Kivell et al., 2014). The regulation of DAT is particularly pertinent to an anti-addictive mechanism of action, and in vitro experimentation has indicated that KOPr-expressing cells will increase DA uptake and cellsurface expression of DAT when exposed to the KOPr agonist Salvinorin A (Kivell et al., 2014). KOPr and DAT are co-expressed in dopaminergic cells of the rat NAc (Svingos et al., 2001), and there is evidence for the formation of KOPr-DAT in cells co-transfected with DAT and KOPr, very likely allowing for direct regulation of DAT by physical association with the KOPr (Kivell et al., 2014). Dual expression of KOPr and DAT is observed in several regions of the brain, in both rats and humans (see Fig. 1.4) (Ciliax et al., 1999; Ciliax et al., 1995; Mansour et al., 1987; Tempel \& Zukin, 1987).The regulatory activity of the KOPr upon DAT may be central to many KOPr-mediated behavioural effects, particularly the negative regulation of reward.

Regulation of SERT by KOPr may also influence drug-associated behaviours. Agonist stimulation of cells co-expressing KOPr and SERT in vitro decreases SERT activity (Kivell et al., 2014), and in vivo studies in 5-HT-depleted rats indicate that 5-HT activity may be integral to depression of the hyperactive locomotor response observed in acute cocainetreated rats pre-treated with chronic KOP agonist (Zakharova et al., 2008), as well as mediating aversive, stress-associated behavioural effects (Bruchas et al., 2011; Land et al., 2009). 


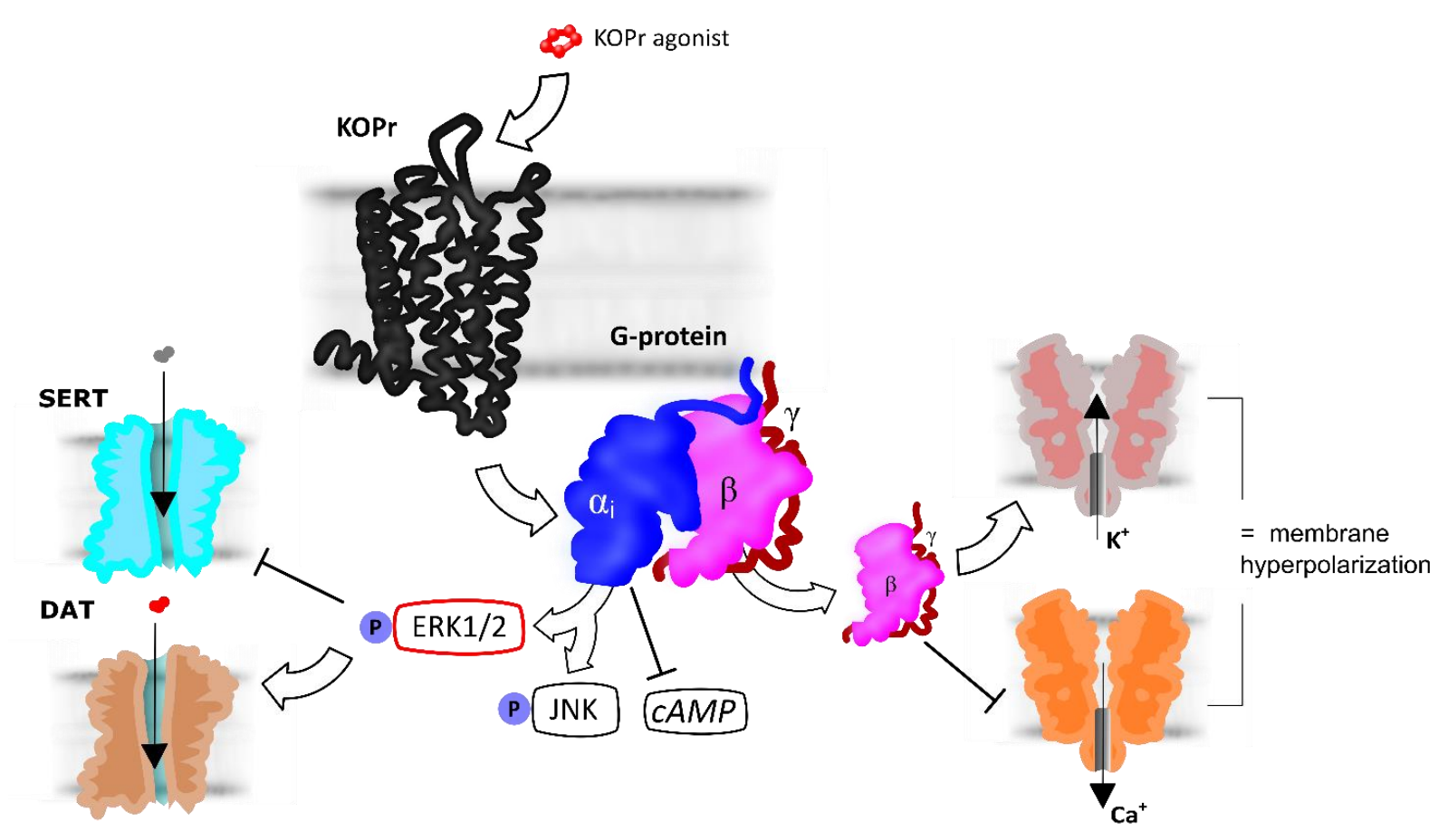

LATE PHASE

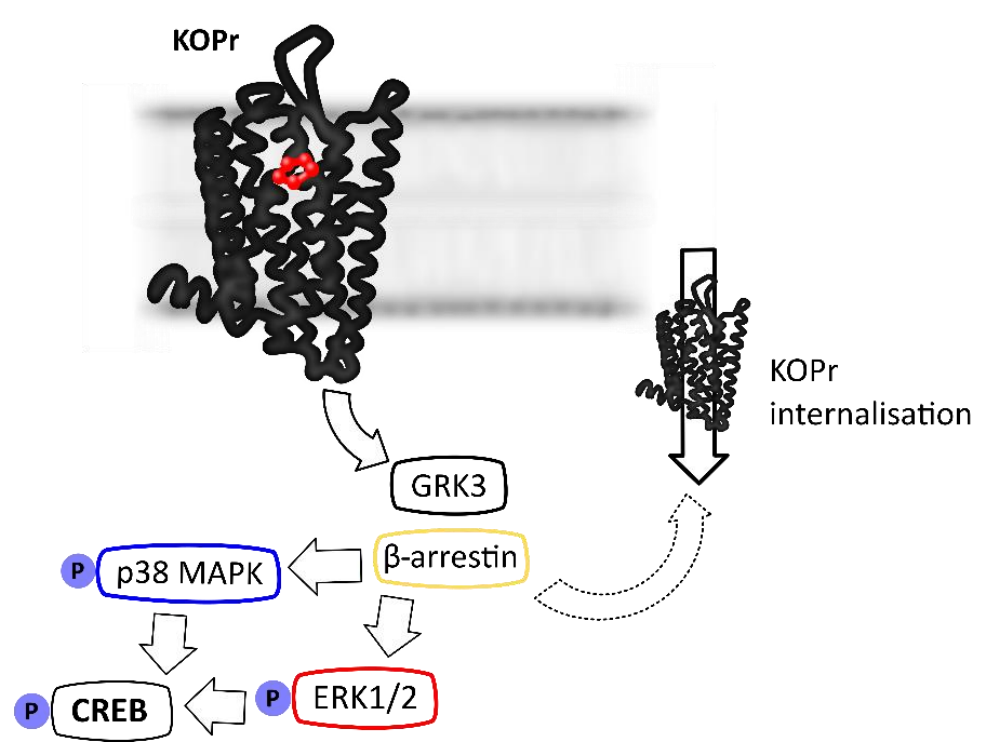

Figure 1.3. Summary of KOPr signalling pathways and effects. An illustration of the early (top) and late (bottom) phases of ERK1/2 activation associated with KOPr activation, including downstream regulatory effects. Arrows indicate activation or stimulation; bluntended lines represent inhibition. Adapted from Bruchas and Chavkin (2010). $c A M P=c y c l i c$ adenosine monophosphate; $C R E B=C A M P$ response element-binding protein; $D A T=$ dopamine transporter; ERK1/2= extracellular signal-regulated kinase 1 and 2; GRK3= Gprotein coupled receptor kinase 3; JNK=c-Jun N-terminal Kinase; p38 MAPK= p38 mitogen-activated protein kinase; SERT= serotonin transporter. 


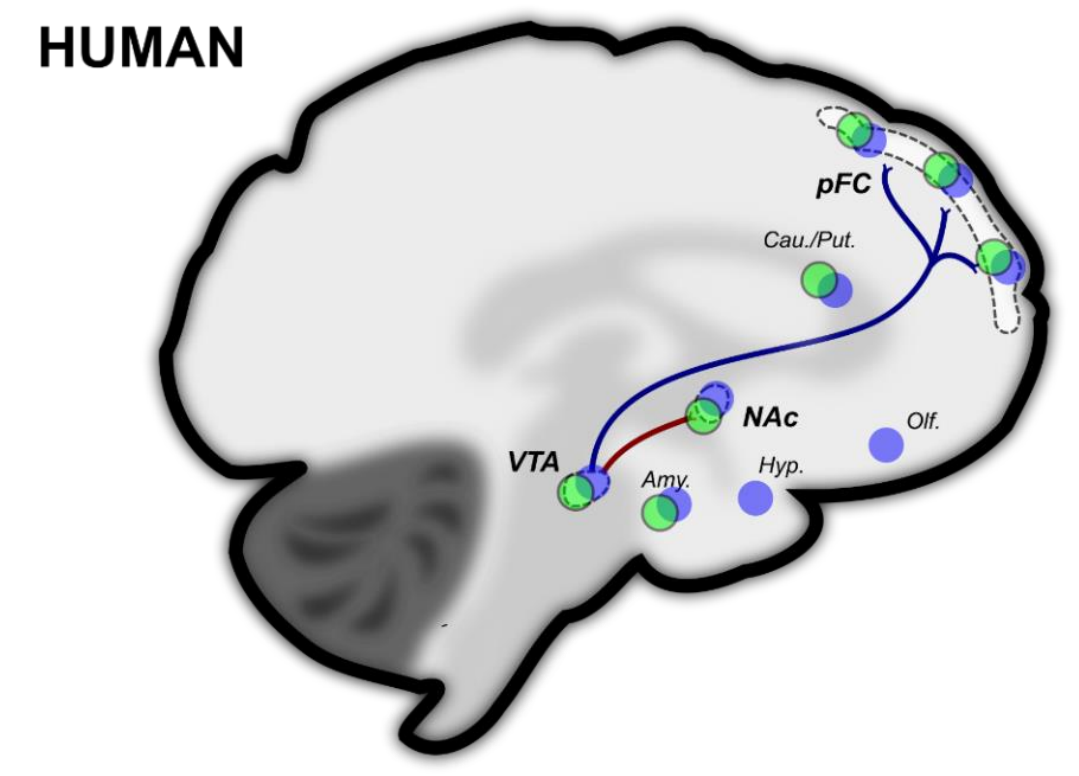

RAT

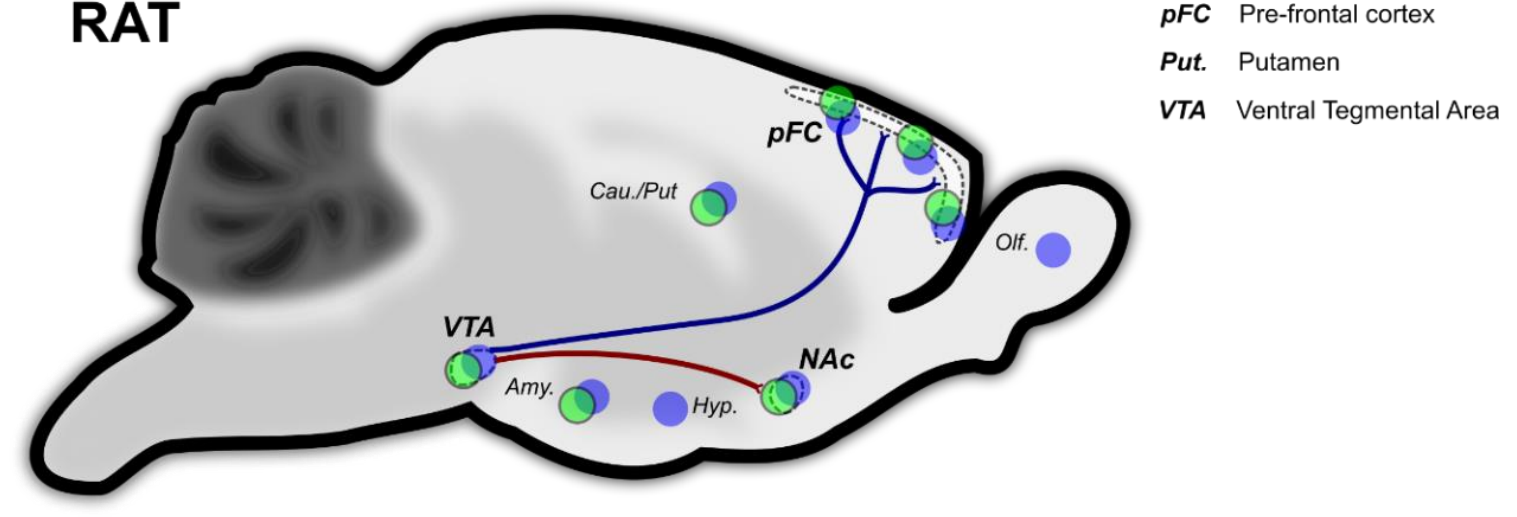

DAT

KOPr

(1) Co-expression

Brain regions:

Amy. Amygdala

Cau. Caudate nucleus

Hyp. Hypothalamus

NAc Nucleus accumbens

Olf. Olfactory bulb

pFC Pre-frontal cortex

Put. Putamen

Ventral Tegmental Area

Figure 1.4. KOPr and DAT co-expression in the brain. Expression profiles of DAT and KOPr in human and rat brain tissue, based upon tissue immunohistochemistry assays by Ciliax et al. (1995,1999), Mansour et al. (1987) and Tempel and Zukin (1987). The size and number of the coloured circles does not represent relative expression. Coexpression of DAT and KOPr is observed in areas associated with reward (VTA, NAc and $\mathrm{pFC}$ ), mood (NAc, amygdala), and learning and memory (caudate nucleus, putamen). Regulation of DAT by KOPr may therefore play an important role in regulating both reward and mood states within the brain, as well as the development of reward association. 


\subsubsection{Kappa-opioids in addiction research}

The use of acute KOPr-agonist treatment for addiction is currently being explored in preclinical trials. Experiments in drug-conditioned rats and mice with synthetic KOPr agonists have demonstrated the ability of KOPr ligands to decrease the rewarding effects of drugs, particularly that of cocaine.

The selective KOPr agonist trans-( \pm )-3,4-Dichloro-N-methyl-N-[2-(1pyrrolidinyl)cyclohexyl]benzeneacetamide (U-50,488; see Fig. 1.5$)$ has been shown to block conditioned place preference (CPP) for cocaine and attenuate cocaine-induced hyperactivity in rats at $5 \mathrm{mg} / \mathrm{kg}$ (s.c.) (Crawford et al., 1995), and reduces cocaine selfadministration (U-50,488 at 0.032-0.1 $\mathrm{mg} / \mathrm{kg} / \mathrm{hr}$, i.v.) in rhesus monkeys (Negus et al.,

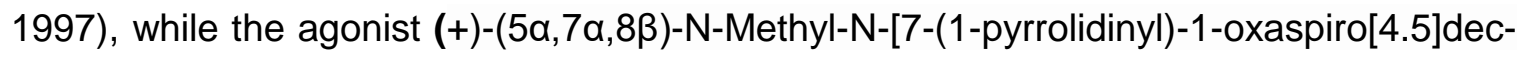
8-yl]-benzeneacetamide (U-69,593; see Fig. 1.5) has been shown to attenuate cocaine but not amphetamine reinstatement of drug-seeking behaviour in rats with 0.16 and 0.32 $\mathrm{mg} / \mathrm{kg}$ (s.c.) pre-treatment (Schenk et al., 1999). Pre-treatment with either U-69,593 (0.04$0.32 \mathrm{mg} / \mathrm{kg}$, s.c.) or U-50,488 $(2.5-7.5 \mathrm{mg} / \mathrm{kg}$, s.c.) has also been shown to block the development of behavioural sensitisation to cocaine in rats in a chronic administration model (Heidbreder et al., 1995). A study by Morani et al. (2009) established that treatment with the agonists U-50,488 (30 mg/kg, i.p.), U-69,593 (0.3 mg/kg, s.c.) and spiradoline (1 $\mathrm{mg} / \mathrm{kg}$, i.p.) in drug-conditioned rats resulted in significant dose-dependent decreases in drug-seeking behaviour using a cocaine self-administration model. Pre-treatment with spiradoline and $\mathrm{U}-50,488$ has also been shown to attenuate responses during standard cocaine and morphine self-administration sessions (Glick et al., 1995). KOPr agonists are therefore promising potential leads for anti-addictive therapies (Prisinzano et al., 2005). A self-administration study by Freeman et al. (2014) showed that infusions of the selective KOP agonist Salvinorin A (SalA) co-infused with either cocaine or the MOR agonist remifentanil could reverse lever preference in rhesus monkeys, indicating that KOP agonists can act as "punishers" of drug-seeking behaviour. Classical synthetic KOPr 
agonists, however, cause a variety of un-wanted side-effects in both human and animal models, including dysphoria, sedation, and nausea which limits their clinical applications (Land et al., 2008; Mello \& Negus, 2000; Pfeiffer et al., 1986).

Chronic KOPr agonist administration has also been shown to paradoxically increase druginduced self-administration behaviour (Negus, 2004; Potter et al., 2011), an effect which may be linked to the proposed role of the KOP system in the homeostatic "opponent process theory' of drug abuse. Opponent process theory proposes that the initial rewarding 'high' provided by drugs of abuse is followed by a reciprocal aversive and dysphoric state (Solomon \& Corbit, 1974), a process which is specifically induced in response to hyperactivity of the reward pathway (Vargas-Perez et al., 2007). In drug-dependant individuals, it is theorised that pro-aversive KOPr signalling out-weighs the initial reward response, enhancing withdrawal symptoms and reinforcing the ability of the drug of abuse to temporarily alleviate the dysphoric withdrawal state (Walker et al., 2012). Therefore, chronic exposure to KOP agonists may exacerbate the withdrawal state and enhance dependence upon the drug of abuse, by facilitating negative reinforcement of drug-taking behaviour (Wee \& Koob, 2010). This theory is supported by animal studies of alcohol abuse where treatment with the long-acting kappa opioid antagonist norbinaltorphimine (norBNI; see Fig. 1.5) at 15 and $20 \mathrm{mg} / \mathrm{kg}$ was shown to reduce ethanol self-administration in ethanol-dependant rats (Walker et al., 2011). KOP antagonism in these animals is proposed to block at least some of the negative reinforcing effects of ethanol abuse and so reduce their dependence on the drug (Walker et al., 2012). This effect has also been shown in models of cocaine and methamphetamine administration, where treatment with norBNI (15 and $30 \mathrm{mg} / \mathrm{kg}$ ) prevented the escalation in dosage normally observed in rats allowed to administer cocaine during "long-access" 6 hour sessions (Wee et al., 2009; Whitfield et al., 2015). These studies therefore indicate that KOP antagonism may also hold promise as a preventative or protective anti-addictive therapy. 
<smiles>C[C@H](C1CCC2(CCCO2)C[C@H]1N1CCCC1)N(C)C(=O)Cc1ccc(Cl)c(Cl)c1</smiles><smiles>CN(C(=O)Cc1ccc(Cl)c(Cl)c1)[C@@H]1CCCC[C@H]1C1CCCC1</smiles><smiles>Cc1ccccc1CC(=O)C(C)[C@H]1CCC2(CCCO2)C[C@H]1N1CCCC1</smiles>

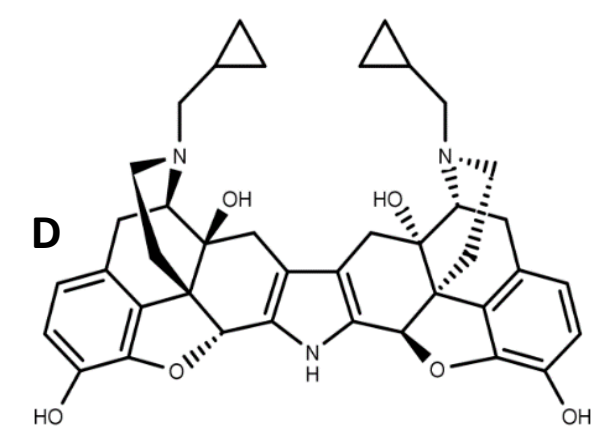

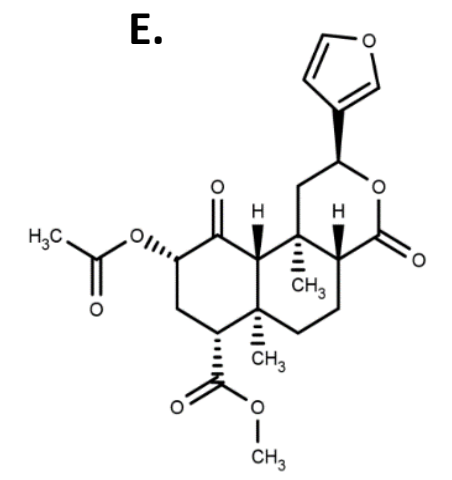

Figure 1.5. KOPr agonists and antagonist. Molecular structures of the synthetic alkaloid KOPr agonists spiradoline (A), ( \pm U- $50,488(B)$, and U-69,593 (C), and the selective KOPr antagonist norBNI (D). For comparison, the non-nitrogenous structure of neoclerodane diterpene Salvinorin A $(E)$ is shown [structures generated using MarvinSketch chemical drawing software]. 


\subsection{Novel Kappa-opioid Agonists}

\subsubsection{Functional Selectivity}

It has been identified that many receptors, in particular the GPCRs, are not limited to a single functional profile. Studies of the down-stream signalling effects of different ligands upon a single receptor have shown that certain ligands can favour the activation of one or more intracellular signalling pathways over others, an effect which is known as functional selectivity or 'ligand bias' (Kenakin, 2011). Functionally selective ligands have been identified for the mu-opioid receptor (MOR) which do not promote MOR internalisation (Groer et al., 2007) and display a reduced side-effect profile compared to classical MORagonists (DeWire et al., 2013), characteristics which may be correlated with decreased $\beta$ arrestin recruitment. The identification of functionally selective agonists for the KOPr which avoid or minimise $\beta$-arrestin recruitment and late-phase ERK1/2 activation offer the most promising means of developing clinically viable KOPr-based therapies (Le Naour et al., 2014).

\subsubsection{Salvinorin A}

A new source of synthetic KOP compounds has stemmed from the discovery of the potent and selective KOPr-agonist properties of Salvinorin A (SalA), the active component of the hallucinogenic plant Salvia divinorum. Salvia has a history of use in traditional and religious ceremonies by the Mazatec Indians of Oaxaca, Mexico (Valdes, 1983), and the plant has gained some recent popularity as a recreational drug due to its hugely potent hallucinogenic effects (Lange et al., 2008). Originally identified structurally by Ortega et al. (Ortega et al., 1982), SalA has been shown to selectively activate KOPrs without interaction at 5-HT receptors, unlike other hallucinogens such as lysergic acid diethylamide (LSD) (Butelman et al., 2007; Roth et al., 2002), and is not self-administered 
even at low doses by rats (Serra et al., 2015). SalA has therefore been used as a lead compound for the manufacture of novel anti-addictive (Prisinzano et al., 2008) and analgesic pharmacotherapies (McCurdy et al., 2006).

SalA is unusual amongst other known KOPr agonists in that its structure carries no nitrogen, as the presence of a charged nitrogenous group was once thought to be a universal feature of opioid agonists (Bera \& Ghoshal, 2014). As with other KOP agonists, the anti-addictive action of SalA likely occurs through attenuation of DA release in the NAC (Ebner et al., 2010) and stimulation of enhanced DAT activity (Kivell et al., 2014). The compound shows a strong reduction in drug-seeking behaviour in cocaine-conditioned rats at a dosage of $0.3 \mathrm{mg} / \mathrm{kg}$ (Morani et al., 2009) without decreasing spontaneous movement (i.e. causing sedation) or causing taste aversion (Morani et al., 2012). In vitro, SalA displays an approximate 40-fold reduction in $\beta$-arrestin-associated KOPr internalisation compared to U-50,488 (Wang et al., 2005), which suggests that the ligand may display some degree of functional selectivity.

Though SalA appears to show anti-depressive effects in mice and rats when given at very low doses of $0.001-1000 \mu \mathrm{g} / \mathrm{kg}$ (Braida et al., 2009) and induces rewarding effects in zebrafish when administered at 5 and $10 \mu \mathrm{g} / \mathrm{kg}$ (Braida et al., 2007), SalA has been shown to cause depressive-like effects in rats subjected to the forced swim test (FST) at $0.3 \mathrm{mg} / \mathrm{kg}$ (Carlezon et al., 2006; Morani et al., 2012). In addition to pro-depressive effects at high doses, SalA is rapidly metabolised and has a very short duration of action (Hooker et al., 2008) making the compound unsuitable for therapeutic use. SalA is a substrate of the blood-brain barrier transporter P-glycoprotein ATPase (Teksin et al., 2009), and Pglycoprotein activity is likely a major contributor to the fast clearance of SalA from the brain (Butelman et al., 2012). SalA has been used as the basis for the creation of a number of synthetic KOPr agonists (Prevatt-Smith et al., 2011) which seek to retain the selectivity and anti-addictive effects of the parent compound, with improved pharmacokinetic parameters and reduced side-effects. 


\subsubsection{Binding Interactions with the KOPr}

A number of binding models have been proposed for the interaction of SalA with the KOPr, with the most current, by Kane et al. (2008), concluding that SalA binds in a region between Transmembrane II and VII of the KOPr, with residues $\mathrm{Y}$ (tyrosine) 119, Y313, Y320 and Q (glutamine) 115 of the KOPr identified as important mediators of SalA binding (see Fig. 1.6 for summary). Agonist binding is most likely enabled through a mixture of hydrogen bonding (Q115), hydrophobic Van der Waals (Y119 and Y313) and aromatic 'pi-stacking' (Y320) interactions (Kane et al., 2008). The furan ring of SalA has been identified to be particularly crucial for high-affinity agonist binding, as the KOPr binding site appears to have very low tolerance for chemical substitution of the furan ring (Harding et al., 2006; Simpson et al., 2007) and its substituents (Riley et al., 2014). Alteration of these sites tends to greatly decrease agonist binding affinity, suggesting that availability of the Y320 region of the KOPr binding site is dependent upon steric agonist-receptor interactions (Riley et al., 2014). An isomer of SalA with alteration to the position of the furan ring, 12 epi-Salvinorin A, also displayed reduced binding affinity compared to unmodified SalA (Béguin et al., 2009), giving further evidence for a highly specific binding site.

The KOPr is more tolerant of modifications to other carbon positions of the SalA molecule. Chemical addition to the carbon-2 position of SalA have produced two analogues with enhanced KOPr-binding affinity, (Munro et al., 2008; Wang et al., 2008), and several analogues with similar binding affinities to the parent compound (Prevatt-Smith et al., 2011; Simonson et al., 2015). 


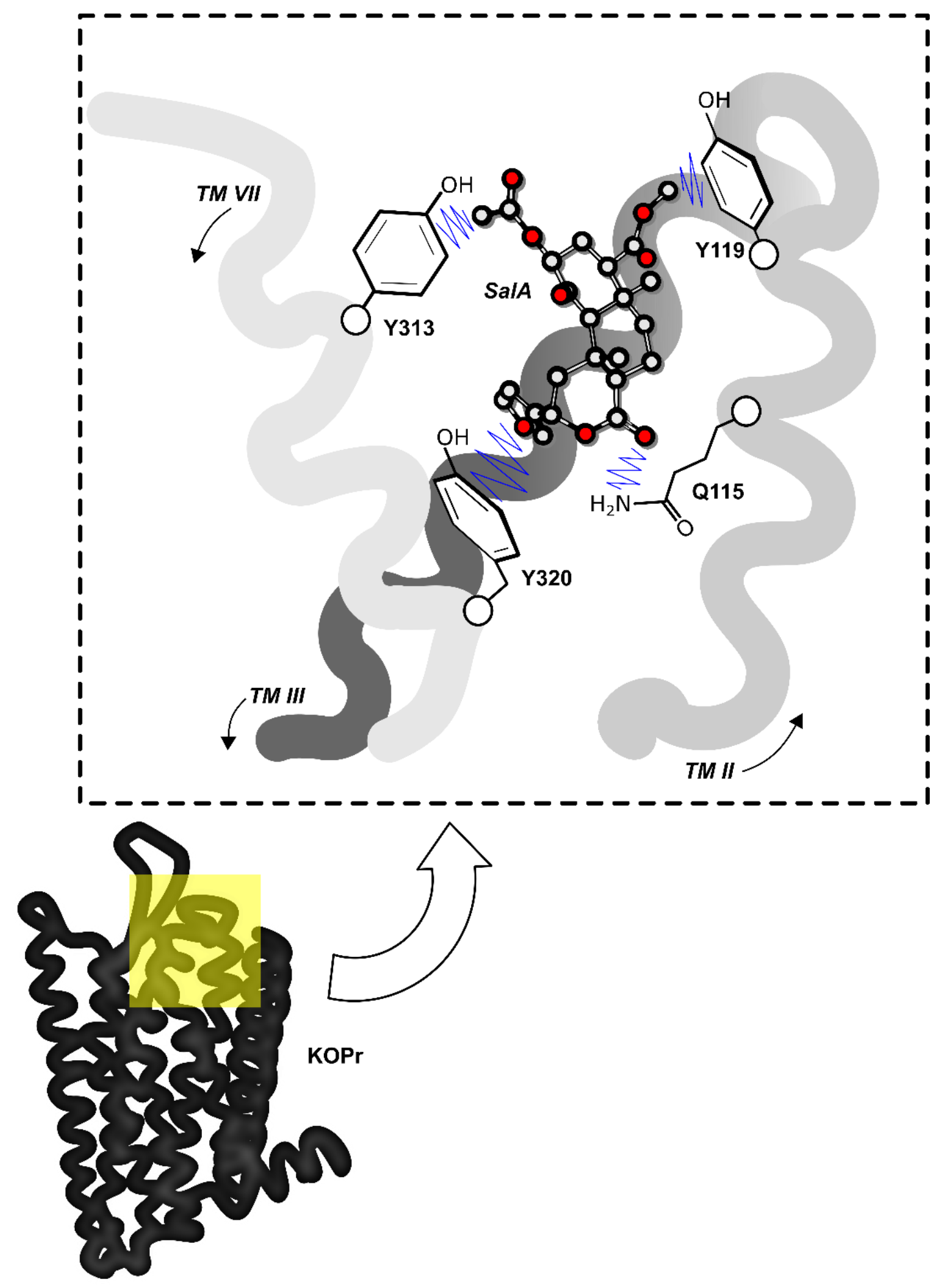

Figure 1.6. SalA binding interactions with the KOPr. Adapted from Kane et al. (2008). Red circles represent oxygen atoms; grey circles are carbon atoms (SalA); blue lines represent proposed binding interactions between labelled residues and SalA groups. $T M=$ transmembrane region. KOPr crystal structure adapted from Wu et al. (2012). 


\subsubsection{Synthesis of novel Salvinorin-based KOPr agonists}

The synthesis of the novel KOPr agonists used in this thesis has been performed by the laboratory of Professor Thomas Prisinzano (Kansas University) (Harding et al., 2006), who has guided the creation of a series of compounds which preserve the basic structure of Salvinorin A but contain functional changes to the acetate group at the carbon-2 position. Alterations at this position have the potential to increase potency and binding affinity at the KOPr (Prevatt-Smith et al., 2011), and allow for the addition of metabolism-protective functional groups (Munro et al., 2008). Transformation at the carbon-2 position has yielded the SalA analogues 2-methoxymethyl (MOM) SalB, 2-ethoxymethyl (EOM) SalB, (Morani et al., 2013; Munro et al., 2008), Mesyl-SalB (Harding et al., 2005; Simonson et al., 2014), and $\beta$-tetrahydropyran ( $\beta$ THP) SalB (Prevatt-Smith et al., 2011), all of which have been confirmed to act selectively at the KOPr. Self-administration studies have also shown that these compounds all act to reduce drug-seeking behaviour in cocaine reinstatement tests with rats (see $\underline{\text { Table 1.1) }}$. The analogue MOM-SalB also appears to display some degree of functional selectivity, by activating only early-phase ERK1/2 (McLennan et al., 2008). However, at least one of these compounds (MOM-SalB) displays depressive side effects in rat models (Morani et al., 2013) and there is a need for further anti-addictive SalA analogues with reduced side-effects.

A novel set of SalA-based compounds have recently been generated through cycloaddition to the carbon-16 position of the furan ring present on SalA (Riley et al., 2013). Three of these compounds have been made available to our laboratory: 16-bromo Salvinorin A (Br-SalA), 16-methyl Salvinorin A (Me-SalA) and 16-ethynyl Salvinorin A (Ethy-SalA). Br-SalA has been shown to attenuate cocaine-seeking behaviour at a dosage of $1 \mathrm{mg} / \mathrm{kg}$, and appears to display few negative side effects in preliminary behavioural studies (A. Ewald, PhD thesis 2015). Ethy-SalA and Me-SalA have been screened for activity at the KOPr and have been identified as KOPr agonists with no activity at the MOR (Riley et al., 2014), but have yet to be tested in vivo. 


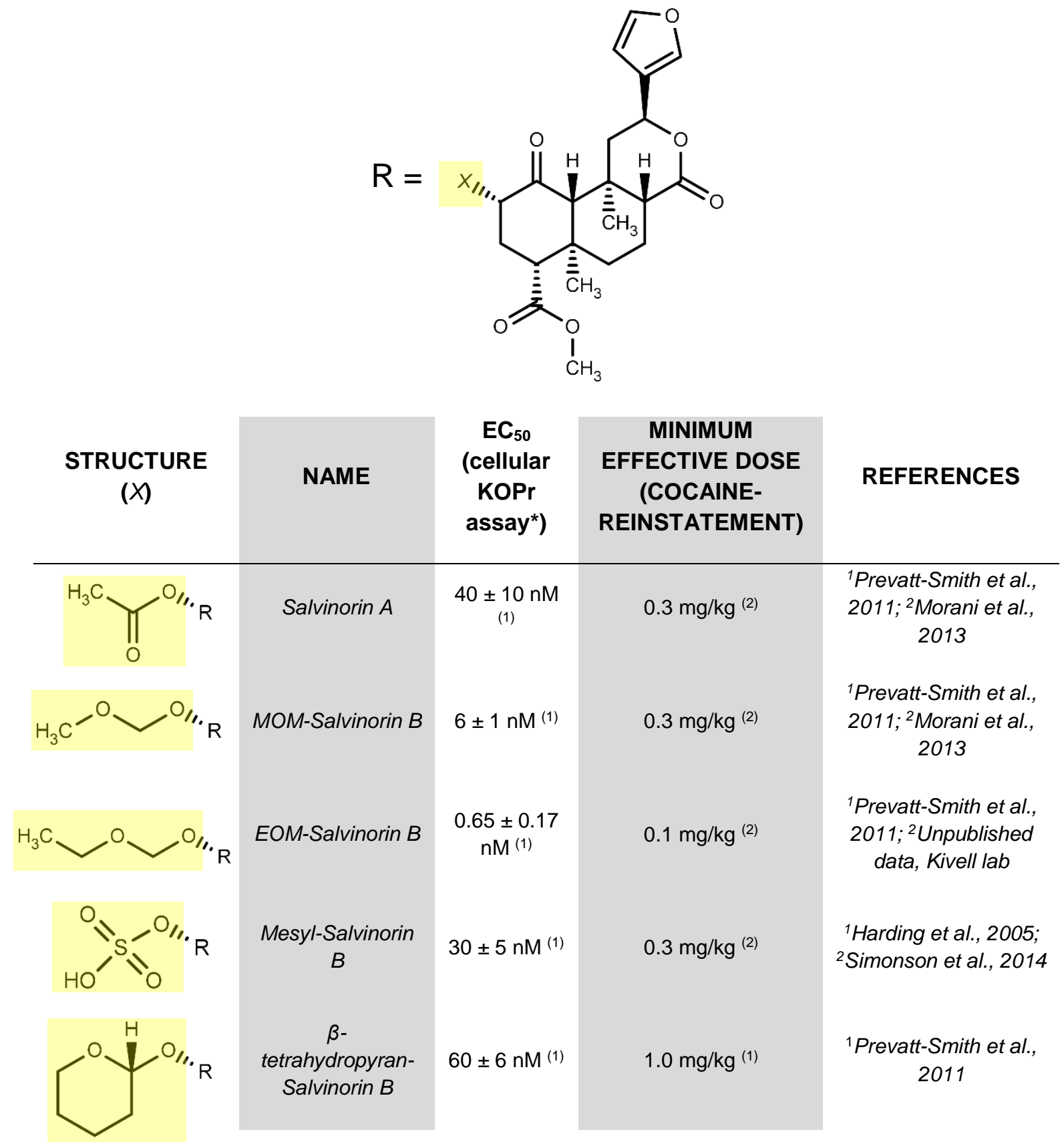

Table 1.1. Carbon-2 analogues of SalA. Chemical structures and comparative potencies (EC50) of Salvinorin A and analogues arising from modification to the functional group of carbon-2. All effective doses are i.p. injections of compound in rats. * Assays for potency at the KOPr were performed using a [35S]GTP-y-S functional assay; the effective concentration (50\%) is in comparison to the stimulation of [35S]GTP- $y-S$ binding to the KOPr by U-50,488 (500 nM). Assays were performed in Chinese Hamster Ovary (CHO) cells stably expressing KOPr. 


\subsection{Aims and Hypotheses}

This thesis aims to investigate the potential anti-cocaine effects of an acute administration of the novel KOPr agonists 16-ethynyl Salvinorin A and 16-methyl Salvinorin A in models of cocaine self-administration, cocaine-primed reinstatement of drug-seeking behaviour and cocaine-induced hyperactivity and behavioural sensitisation. We hypothesise that these compounds will display a similar anti-cocaine profile to the Salvinorin A in cocaineprimed reinstatement and cocaine sensitisation and hyperactivity behavioural tests. This thesis will also evaluate the effects of acute KOPr agonist administration in a progressiveratio model of cocaine self-administration, as well as the behavioural side-effects including appetite and natural reward suppression, sedation, and depressive-like behaviours. We also aim to partially explain the mechanism of action of these novel compounds by testing their ability to regulate DAT function in a cellular model of DA reuptake. 


\section{Chapter 2 : Behavioural anti-cocaine effects of novel KOPr agonists}

\subsection{Methods}

Multiple self-administration and locomotor assays were used to determine the ability of acute pre-treatments of Ethy-SalA and Me-SalA to alter characteristic behaviours associated with cocaine administration in rats. These behavioural tests were designed to detect the potential anti-cocaine effects of these novel KOPr agonists, as first step to identifying a KOPr-based anti-addictive pharmacotherapy. Self-administration procedures were used as models of active cocaine-seeking and taking behaviours in rats, while cocaine hyperactivity and behavioural sensitisation tests were used as secondary measures of anti-cocaine effects.

\subsubsection{Animals}

Male Sprague-Dawley rats were used for all behavioural testing, and were bred and housed within a secure facility at the School of Biological Sciences in Victoria University of Wellington with a 12-hour light/dark cycle (light on at 0700 hours, dark at 2100 hours), or were obtained from a similar facility operated by the School of Psychology. Rats were housed in white plastic cages within a shelved Scantainer Ventilated Cabinet (Scanbur Technology, Karlslunde, Denmark) controlled for temperature $\left(19-21^{\circ} \mathrm{C}\right)$ and humidity (55\%). All rats were housed in groups of $2-4$ rats per cage, excepting cocaine selfadministration rats, which were housed individually. Rats used for sucrose selfadministration rats weighing between 250-350 g were housed two to a cage, and were fed a restricted diet to maintain approximately $85 \%$ of their initial weight, but had ad libitum access to water (except when undergoing self-administration training or testing). All other 
rats were given ad libitum access to both food and water. All behavioural experimental work was carried out between 0800 and 1600 hours, in the presence of white noise or with sound-attenuating boxes. All experimental procedures were approved by the Victoria University of Wellington Animal Ethics Committee (Ethics Approval AEC-2012-R34). Rats used for self-administration and behavioural sensitisation were cocaine naïve prior to surgery. Rats used for locomotion assays and the Forced Swim Test were re-used from prior experimentation, after a minimum of a week's rest period, in accordance with the recommendation of the National Centre for the Replacement, Refinement and Reduction of Animals in Research (UK) (Russell \& Burch, 1959).

\subsubsection{KOPr agonists}

All SalA-derived KOPr agonists were generously provided by Prof. Thomas Prisinzano. SalA was extracted and purified from the leaves of Salvia divinorum (Butelman et al., 2007). The novel chemical analogues of SalA (Ethy-SalA, Me-SalA) were produced using Suzuki-Miyaura coupling to the furan ring of SalA followed by reduction (Riley et al., 2014). All compounds were purified using column chromatography, and solid products were determined to be $>95 \%$ pure using analytical High-Performance Liquid Chromatography (HPLC) (Riley et al., 2014). Powders were stored at $4^{\circ} \mathrm{C}$ in the dark before use.

Unless specified, all KOPr agonists used in this and in other chapters for behavioural assays were dissolved in a vehicle of dimethyl sulfoxide (DMSO), polysorbate 80 (Tween 80) and double-distilled water $\left(\mathrm{ddH}_{2} \mathrm{O}\right)$ in a 2:1:7 ratio. KOPr agonists in powdered form were weighed and completely dissolved in DMSO before adding Tween 80 and $d \mathrm{dd}_{2} \mathrm{O}$, with vortexing to ensure complete mixture of the solution. KOPr agonist solutions were stored at $4^{\circ} \mathrm{C}$ in the dark for a maximum of 1 week. Agonist pre-treatment times and injection methods for Sal A, U-50,488 and U-69,593 were based upon previous literature examining the anti-cocaine effects of these drugs (Morani et al., 2009). 


\subsubsection{Chemicals and solutions}

Stock solutions of pentobarbital $(500 \mathrm{mg} / \mathrm{mL})$, xylazine $(20 \mathrm{mg} / \mathrm{mL})$, ketamine $(100$ $\mathrm{mg} / \mathrm{mL}$ ), carprofen (50 mg/mL), terramycin powder, sodium lactate solution, Vetadine (1.6\% iodine, w/v), heparin $(5,000 \mathrm{unit} / \mathrm{mL})$ and penicillin $\mathrm{G}$ sodium $(1,000,000 \mathrm{IU})$ were purchased from Provet (Auckland, New Zealand). To make heparin solutions of $30 \mathrm{unit} / \mathrm{mL}$ and 3 unit $/ \mathrm{mL}$, the 1,000 unit $/ \mathrm{mL}$ stock was diluted in sterile $0.9 \%$ saline $(0.9 \% \mathrm{NaCl}, \mathrm{w} / \mathrm{v})$ solution, and filtered before use. Working solutions of carprofen ( $5 \mathrm{mg} / \mathrm{mL}$ ) and pentobarbital $(50 \mathrm{mg} / \mathrm{mL})$ solutions were made using sterile, filtered $0.9 \%$ saline solution. Penicillin solution (100,000 unit/mL) was made by dissolving penicillin powder in 30 unit/mL heparin, and is referred to as 'penicillin/heparin solution'.

Cocaine hydrochloride (BDG Synthesis, Wellington, NZ) was dissolved in $0.9 \%$ saline solution containing 3 unit/mL heparin to a concentration of $1.65 \mathrm{~g} / \mathrm{L}$ for cocaine selfadministration (referred to as 'heparinised cocaine'), or in sterile filtered $0.9 \%$ saline solution to a concentration of $20 \mathrm{mg} / \mathrm{mL}$ for cocaine-prime injections.

\subsubsection{Self-administration intra-jugular surgery}

Catheter preparation, intra-jugular surgery procedures and post-operative care are described in detail in Appendix 2. Briefly, rats weighing 300-350 g were anaesthetised with a mixture of ketamine and xylazine (90 mg/kg ketamine, $9 \mathrm{mg} / \mathrm{kg}$ xylazine) and operated upon to insert an indwelling catheter into the right jugular vein. The metal opening of the catheter was fixed on the crown of the head by jewellers screws mounted on the exposed skull and covered with a layer of dental acrylic. The open end of the catheter was sealed with a short, close-ended length of tygon tubing. Rats were monitored for significant weight changes over 5 days, after which self-administration training was begun. Rats were flushed daily with $0.2 \mathrm{~mL}$ of penicillin/heparin solution to prevent blood clots within the 
catheter. Catheter patency was tested weekly by pulling blood back into a syringe from the catheter, or by flushing the catheter with $0.15 \mathrm{~mL}$ of $50 \mathrm{mg} / \mathrm{kg}$ pentobarbital to observe an immediate sedative effect. If a catheter became blocked or developed a leak, rats were further operated upon to replace the blocked metal terminal of the catheter or to introduce a new catheter into the left jugular. If the second catheter was lost, the rat was removed from the experiment and euthanized.

\subsubsection{Cocaine self-administration training}

Self-administration training was performed as previously described (Bosch, 2013). Briefly, training was performed in operant chambers (ENV-001, Med Associates, St. Albans, Vermont) enclosed within sound- and light- attenuating boxes, with a choice of an active (right-hand) or inactive (left-hand) lever paired with a light stimulus. The metal-pieces of the jugular catheters were connected to a $20 \mathrm{~mL}$ syringe of cocaine solution $(1.65 \mathrm{~g} / \mathrm{L})$ within a mechanical pump (PHM-100VS, Med Associates) via tygon tubing Cole-Parmer C-P06418-02, Thermo-Fisher Scientific Inc., Melbourne, Australia). Rats were initially trained under a Fixed-Ratio (FR) -1 schedule, where a single depression of the active lever stimulated activation of the light above the lever and a simultaneous infusion of heparinised cocaine $(0.1 \mathrm{~mL})$. Rats were considered to have acquired drug-seeking behaviour once they were consistently receiving at least 20 infusions over the two hour session, with a ratio of active:inactive lever presses of at least 2:1. After acquisition, rats were moved to an FR-2 schedule (two lever presses per infusion), where they were also required to achieve greater than 20 infusions per session over three days, and were then moved to a FR-5 schedule (5 lever presses per infusion) for a minimum of 10 days. A baseline level of active cocaine responding for each rat was calculated by taking the mean infusions of three consecutive FR- 5 administration sessions, with the variation in response being less than $20 \%$ of the mean. All cocaine infusions and responses and were controlled 
and recorded using Med Associates software (MED-PC IV, version 4.2). Rats were flushed with $0.2 \mathrm{~mL}$ of penicillin/heparin solution immediately prior to and after self-administration sessions. During the training phase, rats were given five consecutive days of selfadministration, followed by two rest days, during which catheters were tested for patency. After establishment of baseline responding, rats were moved to the cocaine-primed reinstatement model.

\subsubsection{Cocaine-primed reinstatement}

Extinction and reinstatement of drug-seeking behaviour was performed using a betweensession design (Bosch, 2013). Once FR-5 responses for heparinised cocaine did not vary by more than $20 \%$ from baseline over two consecutive days, or by more than $10 \%$ over three consecutive days, on the proceeding day the rat was moved to an extinction phase. Cocaine syringes were replaced with a 3 unit $/ \mathrm{mL}$ heparin, $0.9 \%$ saline solution and the light stimulus disconnected from the active lever. Drug-seeking behaviour was judged as being extinguished once active lever responses for heparinised saline dropped below 20 presses over a single 2 hour-session. Rats were then tested on the following day for their ability to reinstate drug-seeking behaviour after a priming injection of cocaine. The light stimulus connection to the active lever was restored, and rats were injected i.p. with cocaine $(20 \mathrm{mg} / \mathrm{kg})$ and placed immediately into the operant chamber with a syringe of heparinised saline available (see Fig. 2.1 for summary). Rats were advanced to the treatment regimen if active lever responses were recorded at or above baseline activity in response to the cocaine prime. For each reinstatement test, rats were required to complete the two-day baseline and extinction phases described above. On the day of reinstatement, rats were pre-treated with Me-SalA $(0.3 \mathrm{mg} / \mathrm{kg}, 1 \mathrm{mg} / \mathrm{kg})$, Ethy-SalA $(0.1 \mathrm{mg} / \mathrm{kg}, 0.3$ $\mathrm{mg} / \mathrm{kg}$ ), SalA $(0.3 \mathrm{mg} / \mathrm{kg}$ ) or vehicle 10 minutes (Me-SalA, Ethy-SalA, vehicle) or five minutes (SalA) before a priming injection of cocaine $(20 \mathrm{mg} / \mathrm{kg})$. Rats were then placed 
into the operant box and allowed to self-administer heparinised saline for 2 hours. The order of treatment was varied using a Latin square design (Appendix 4, Table 6.1) to mitigate the effect of a single treatment order upon the results obtained.

\subsubsection{Progressive-ratio cocaine self-administration}

Rats were trained in cocaine self-administration as previously described (see section 2.1.6) until rats had successfully achieved 10 days at FR-5. Rats were then allowed two further days on a FR5 schedule, before moving to a progressive-ratio (PR) schedule (Fig. 2.2). The schedule used required rats to increase the number of active responses for each new infusion, at a rate calculated using the equation (Roberts, 1993):

Ratio $=\left[\left(5 \times e^{(0.2 \times \text { infusion number })}-5\right)\right]$

Rats were maintained on this PR schedule until the number of infusions achieved on three consecutive days did not vary by more than \pm 2 infusions. On the fourth day, rats were injected with vehicle (i.p.), Ethy-SalA (0.3, 1.0 or $2.0 \mathrm{mg} / \mathrm{kg}$; i.p.), Me-SalA (1.0 or 2.0 mg/kg; i.p.), SalA (at $2.0 \mathrm{mg} / \mathrm{kg}$; i.p.), or U-69,593 (10 mg/kg; s.c.) 10 minutes (vehicle, Ethy-SalA, Me-SalA), 15 minutes $(U-69,593)$ or 5 minutes (SalA) prior to the start of a PR session. The order of treatment was varied using a Latin square design (see Appendix 4 , Table 6.2). After completing a test session, rats were given two days of rest to allow the KOPr agonist to pass out of the rat's system, and to test catheter patency. 


\section{Training}

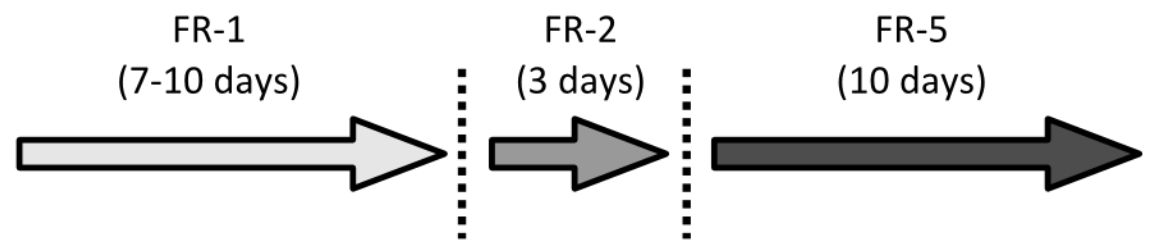

\section{Reinstatement}

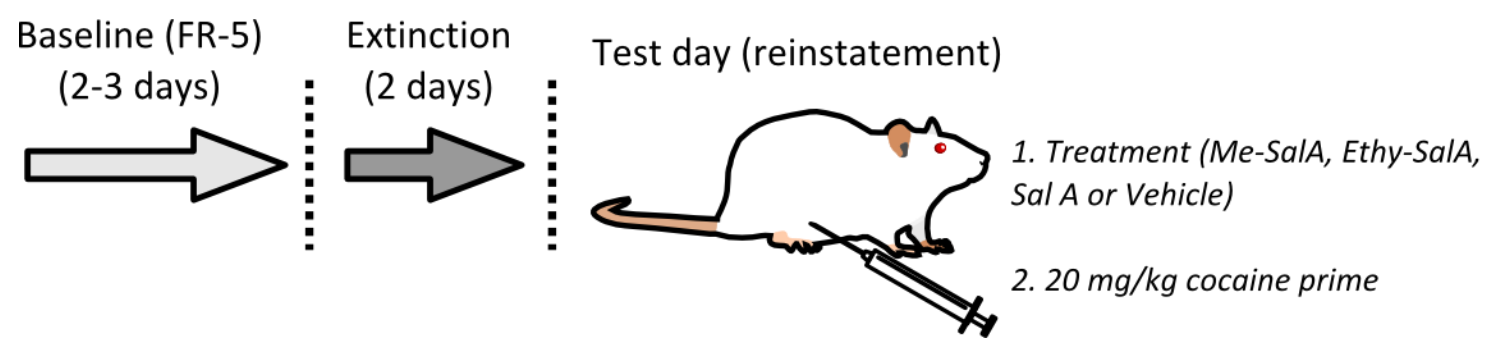

Figure 2.1. Cocaine-primed reinstatement. Schematic of the self-administration training (I.) and reinstatement (II.) procedures used for cocaine self-administration. On the day of reinstatement, treatments were administered 5-10 minutes before the priming injection of cocaine. 


\begin{tabular}{ccc|}
$\begin{array}{c}\text { Infusion } \\
\text { number }\end{array}$ & $\begin{array}{c}\text { Active lever } \\
\text { responses } \\
\text { required }\end{array}$ & $\begin{array}{c}\text { Cumulative } \\
\text { responses }\end{array}$ \\
\hline 1 & 1 & 1 \\
2 & 2 & 3 \\
3 & 4 & 7 \\
4 & 6 & 13 \\
\hline 5 & 9 & 22 \\
6 & 12 & 34 \\
\hline 7 & 15 & 36 \\
\hline 8 & 20 & 69 \\
\hline 9 & 25 & 94 \\
\hline 10 & 32 & 126 \\
\hline 11 & 40 & 166 \\
\hline 12 & 50 & 216 \\
\hline 13 & 62 & 278 \\
\hline 14 & 77 & 355 \\
\hline 15 & 95 & 450 \\
\hline 16 & 118 & 568 \\
\hline 17 & 145 & 713 \\
\hline 18 & 178 & 891 \\
\hline 19 & 219 & 1110 \\
\hline 20 & 268 & 1378 \\
\hline
\end{tabular}

Table 2.1. Progressive-ratio responses. Numbers of required and accumulative responses under the PR self-administration schedule for infusions from 1 to 20.

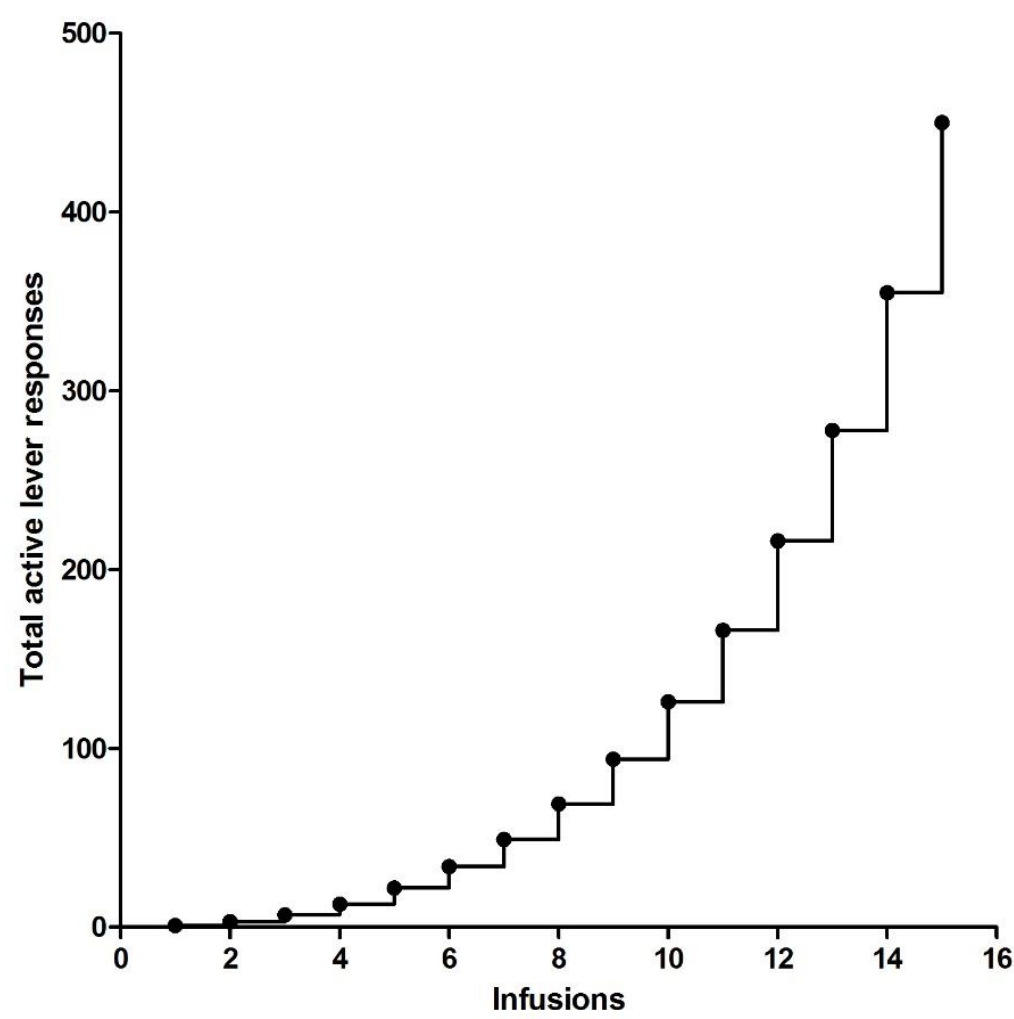

Figure 2.2. Progressive ratio responses. Graphical representation of accumulative responses during a PR self-administration session for one animal up to 15 infusions. 


\subsubsection{Cocaine-induced locomotor hyperactivity}

Cocaine-induced hyperactivity tests were used to explore the ability of acute Ethy-SalA treatment to alter the hyperactive behaviour caused by cocaine in drug-naïve rats, at a dose previously found to attenuate cocaine-reinstatement $(0.3 \mathrm{mg} / \mathrm{kg})$. Rats weighing between 300-400g were placed in open-top activity chambers (ENV-520, Med Associates) within light- and sound-attenuating boxes and allowed to habituate to the space for 30 minutes, before being removed from the chamber and injected with either vehicle (i.p.), $0.3 \mathrm{mg} / \mathrm{kg}$ Ethy-SalA (i.p.) or $0.3 \mathrm{mg} / \mathrm{kg} \mathrm{U}-69,593$ (s.c.) and placed in the home cage for 10 (vehicle, Ethy-SalA treatment) or 15 minutes (U-69,593 treatment). Rats were then removed from the home cage, injected i.p, with $20 \mathrm{mg} / \mathrm{kg}$ cocaine and placed back into the activity chamber and allowed to explore the space for a further 60 minutes. Horizontal movement was detected by infra-red photobeam and transmitters and receivers positioned on the sides of the box, and recorded by Med Associates Activity Monitor Software (SOF811). Stereotypic and ambulatory activity was recorded during both habituation and testing sessions. A single ambulatory count was calibrated to be recorded when three consecutive horizontal photobeam breaks were made by the rat. Stereotypic behaviour was defined by the software as any horizontal movement detected below this threshold.

\subsubsection{Behavioural sensitisation to cocaine}

The cocaine sensitisation assay was used to establish whether acute Ethy-SalA pretreatment $(0.3 \mathrm{mg} / \mathrm{kg})$ could suppress behavioural sensitisation to the locomotorenhancing effects of daily cocaine injections in rats. Drug-naïve rats weighing 300-370 g were obtained from the School of Psychology (Victoria University), and were allowed a period of a week to acclimatise to their new surroundings, and to become used to being handled. Rats were assigned to cocaine- or saline-treatment groups, and received injections of $20 \mathrm{mg} / \mathrm{kg}$ cocaine or saline (i.p.) for five consecutive days. On Day 1, rats 
were first habituated to a locomotor activity chamber (see section 2.1.11) for 30 minutes, then injected with either cocaine or saline and their activity recorded for 60 minutes. On Days $2-5$, rats were injected and placed back into their home cage. Rats received no treatment on days 6-9. On Day 10 of the experiment, cocaine- and saline-treated rats were again habituated to the activity chamber, before being returned to their home cages and injected with either vehicle or $0.3 \mathrm{mg} / \mathrm{kg}$ Ethy-SalA (i.p.), 10 minutes before an injection of $20 \mathrm{mg} / \mathrm{kg}$ cocaine. Rats were then placed back into the activity chamber and locomotor activity was recorded for 60 minutes (see Fig. 2.3).
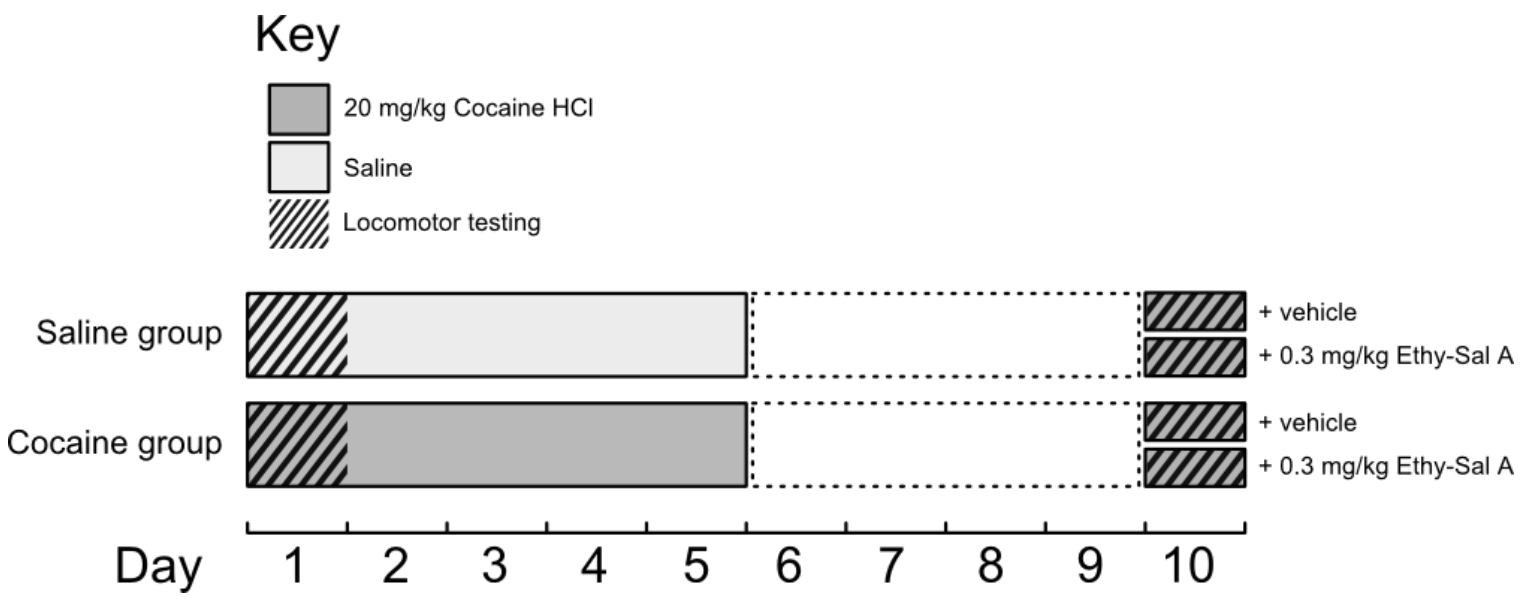

Figure 2.3. Treatment schedule for cocaine sensitisation. Rats were initially split into two treatment groups (Days 1-5), and then into two further groups, receiving either vehicle or Ethy-SalA pre-treatment. 


\subsubsection{Statistical analysis}

All statistical analyses were performed using GraphPad Prism 5.0 software. Rats receiving multiple treatments using a Latin square design (cocaine reinstatement and progressiveratio self-administration) were split into groups and analysed using repeated measures one-way ANOVA with Dunnett's multiple comparison post-test for 3 or more treatment groups, or paired t-tests for 2 treatment groups. For cocaine-reinstatement and progressive-ratio experiments, not all subjects received all treatments, and so for repeated-measures analysis subjects were grouped and analysed separately. Progressive-ratio data was analysed with respect to infusions rather than active responses, as comparisons of exponentially increasing lever responses would violate assumptions of equal variance when performing ANOVA analysis (Richardson \& Roberts, 1996). Results were considered to show significance if the $p$-value for statistical tests was $<0.05$. Numerical results are reported in-text as Mean \pm Standard Error of Mean. 


\subsection{Results}

\subsubsection{Operant training: cocaine self-administration}

All rats used in this experiment acquired cocaine-seeking behaviour within three weeks of the start of cocaine self-administration training (Fig. 2.4A). The shortest time to acquire drug-seeking behaviour was from the first day of administration (1 rat), and the longest time taken was 16 days. All rats, regardless of the length of training, eventually showed significant differences in active, cocaine-delivering lever and inactive lever responses $[F(9,114)=4.91, p<0.0001]$, with active responses observed as being significantly greater than inactive responses over at least three consecutive days [Bonferroni post-test, $p<0.001$ ] (Fig. 2.4B).

Baseline active responses and cocaine administration were monitored to test the assumptions that (1) rats would stably administer cocaine during baseline sessions between reinstatement tests, and (2) that drug-seeking behaviour was consistently extinguished under saline administration condition.

Mean active and inactive lever responses showed significant differences prior to all reinstatement sessions $[F(1,40)=464.50, p<0.0001]$, with inactive significantly less than active responses at each time point [Bonferroni post-test, $p<0.001$ ] (Fig. 2.5A). Mean active and inactive responses were also significantly different during extinction phases $[F(1,40)=26.60, p<0.0001]$, but only prior to the $1^{\text {st }}$ and $6^{\text {th }}$ reinstatement sessions [Bonferroni post-test, $p<0.05$ ]. (Fig. 2.5B). Two-way ANOVA analysis of active responses under baseline and extinction conditions (Fig. 2.5C) identified a significant effect of The administration phase (baseline or extinction) had a significant effect upon active responding $[F(1,40)=429.13, p<0.0001]$, with responses during extinction phases being consistently reduced to approximately $5 \%$ that of baseline-phase responses [Bonferroni post-test, $p<0.001]$. 
Cocaine administration during the baseline phases of each reinstatement course was stable (Fig. 2.6), with linear regression modelling indicating no significant positive or negative correlation between reinstatement number (time) and dose [Spearman's coefficient, $\left.r^{2}=0.0001\right]$. The slope of this regression was not significantly non-zero $[F(1,74)=0.007, p>0.05]$. 
A

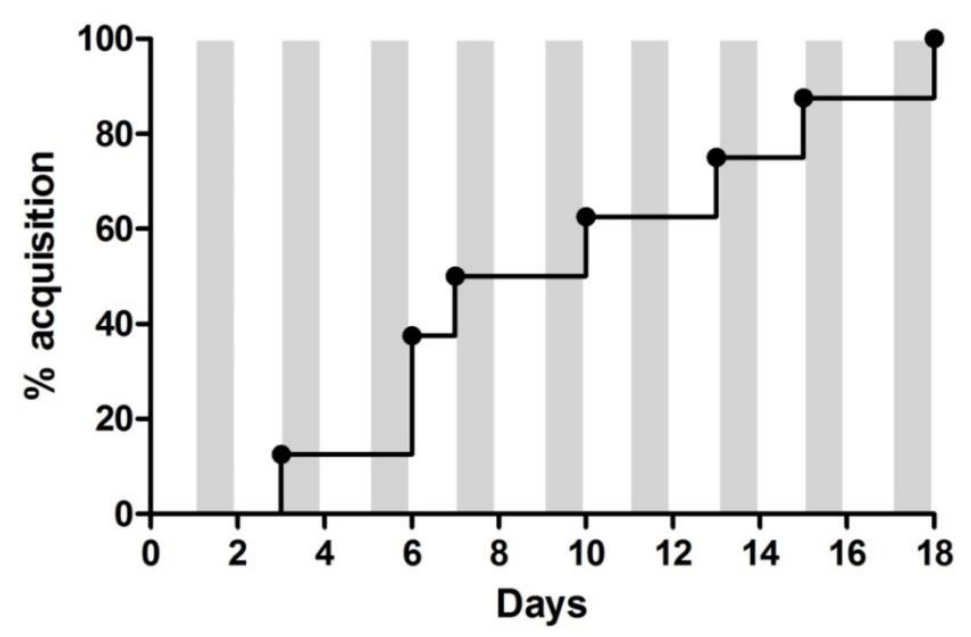

B

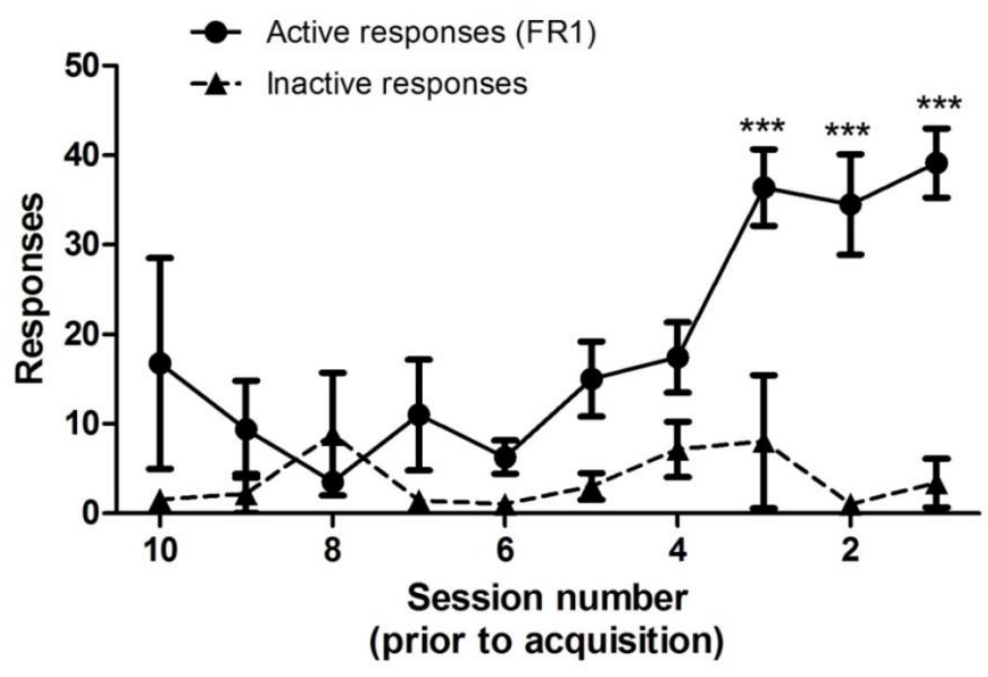

Figure 2.4. Acquisition of cocaine self-administration. All rats used in this experiment acquired cocaine-seeking behaviour within 18 days of the start of cocaine selfadministration training (A). The shortest time to acquire drug-seeking behaviour was from the first day of administration (1 rat). Analysis of active and inactive lever responses (B) shows a highly significant difference between the numbers of active and inactive lever responses for cocaine in all rats over last three days prior to the end of FR1 training. Mean active responses over these three days were $36.67 \pm 5.35$. ${ }^{* *}=p<0.001$ (two-way ANOVA with Bonferroni post-test). 

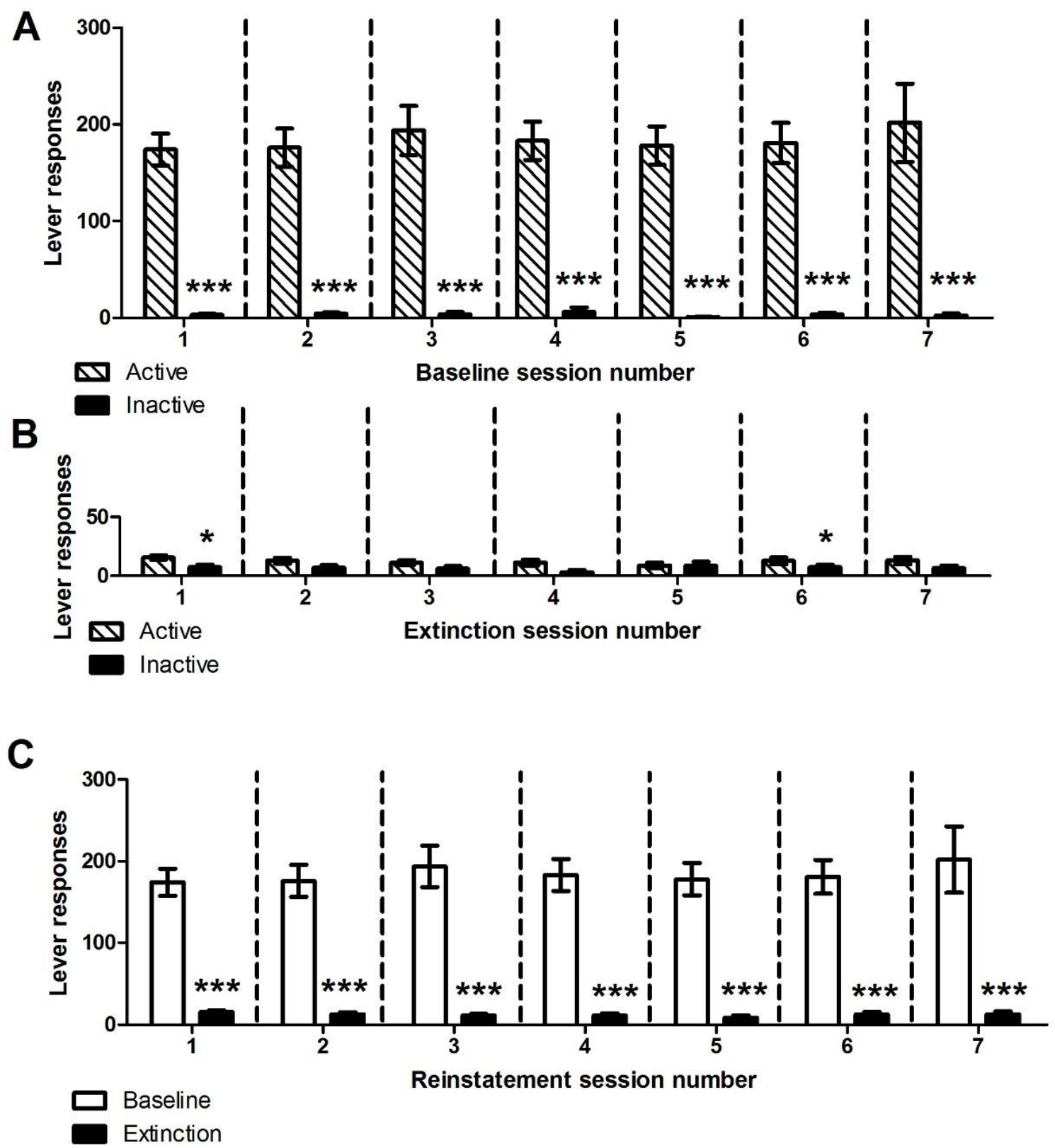

Figure 2.5. Baseline cocaine self-administration responses. Mean active responses during baseline cocaine-administration sessions were significantly greater than inactive responses $(A)$. During the extinction phase, mean active responses did not show significant differences to inactive responses, except during extinction sessions 1 and 6 (B). A comparison of baseline and extinction phases showed highly significant differences between baseline and extinction active responses $(C) .{ }^{*}=p<0.05,{ }^{* * *}=p<0.001$ (two-way repeated measures ANOVA, with Bonferroni post-test). $n=4-8$ rats. 


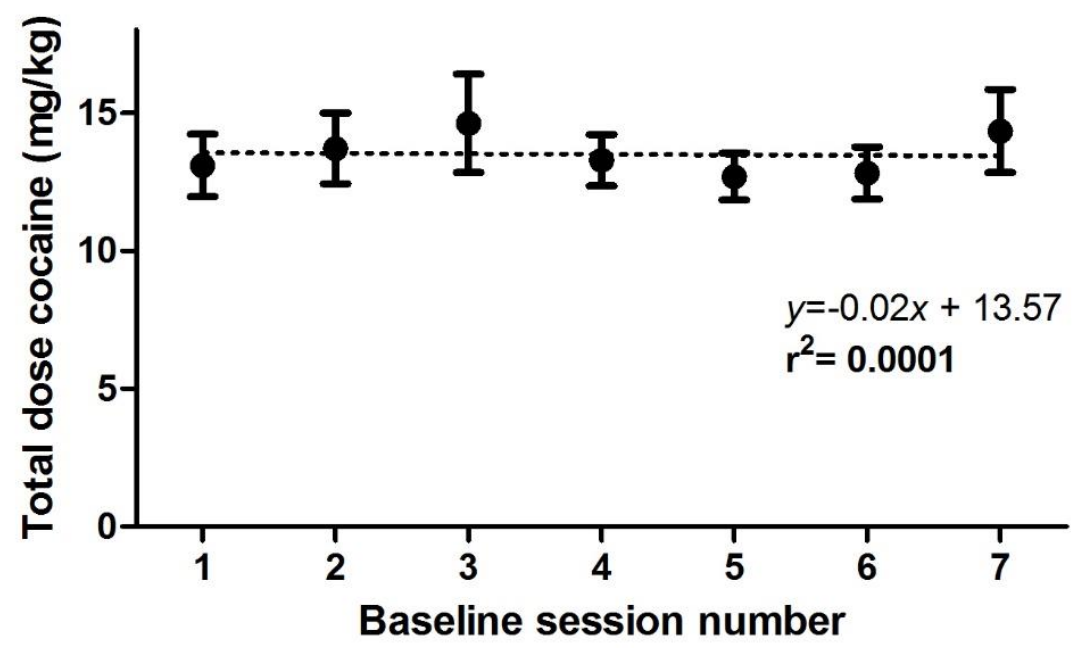

Figure 2.6. Baseline cocaine dose. The cumulative dose of cocaine self-administered during the baseline phases of each reinstatement test was stable, with no significant increase or decrease in dose observed between sessions ( $p>0.05$; one-way ANOVA, with Bonferroni post-test), or over a period of seven reinstatement courses (Pearson correlation coefficient, $r 2=0.0001) \cdot n=4-8$ rats. 


\subsubsection{Cocaine-primed reinstatement}

The mean active lever responses during the initial baseline, extinction and reinstatement phase of cocaine-primed reinstatement (no pre-treatment) testing showed highly significant differences $[F(2,6)=11.87, p<0.01]$. Mean active responses across all extinction phases $(12.48 \pm 1.32)$ were significantly reduced in comparison to mean baseline responses $(190.3 \pm 15.22)$ [Dunnett's multiple comparison test to baseline, $p<0.05$ ]. Activelever responses were restored to approximately baseline levels of responding $(282.0 \pm$ 63.71) $[p>0.05]$ with a cocaine prime $(20 \mathrm{mg} / \mathrm{kg})$ (Fig. 2.7A).

Rats pre-treated with Ethy-SalA or vehicle showed significant differences in their reinstated mean active responses $[F(2,5)=5.23, p<0.05]$. Pre-treatment with $0.1 \mathrm{mg} / \mathrm{kg}$ Ethy-SalA (163.3 \pm 22.79 responses) and $0.3 \mathrm{mg} / \mathrm{kg}$ Ethy-SalA (161.7 \pm 18.18 responses) reduced the number of active responses in comparison to vehicle pre-treatment $(267.8 \pm 41.36$ responses) [Dunnett's multiple comparison test to vehicle, $p<0.05$ ] (Fig. 2.7C). No significant differences were detected between Me-SalA (0.3 and $1.0 \mathrm{mg} / \mathrm{kg}$ ) and vehicle pre-treatment $[F(2,4)=1.95]$. Pre-treatment with SalA significantly attenuated mean lever responses at a dosage of $0.3 \mathrm{mg} / \mathrm{kg}(124.4 \pm 45.60)$ in comparison to vehicle responses (280.4 \pm 48.26) [paired t-test, $t(4)=3.423, p<0.05]$ (Fig. 2.7B) . 


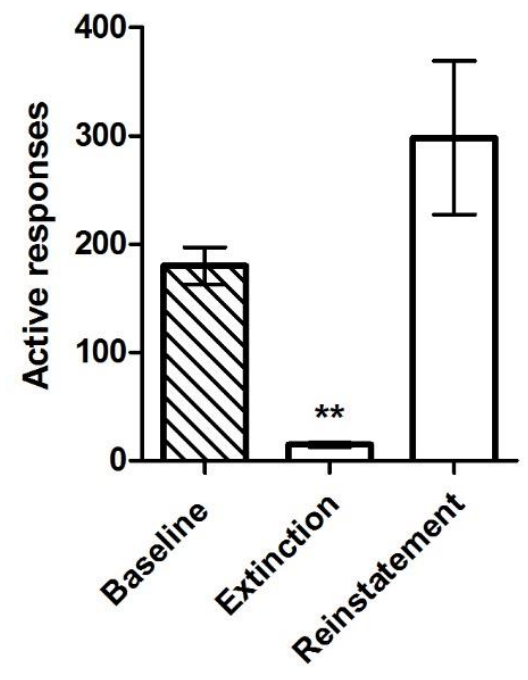

Reinstatement test phase
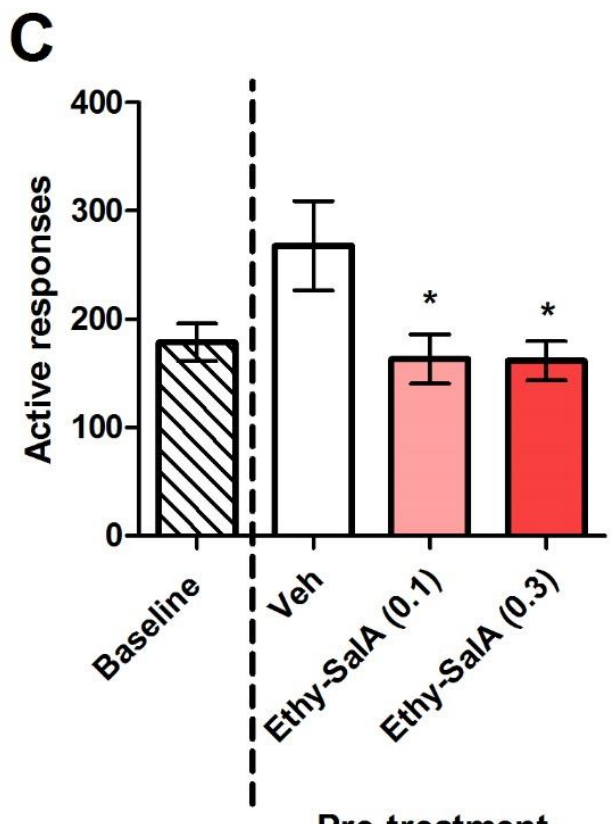

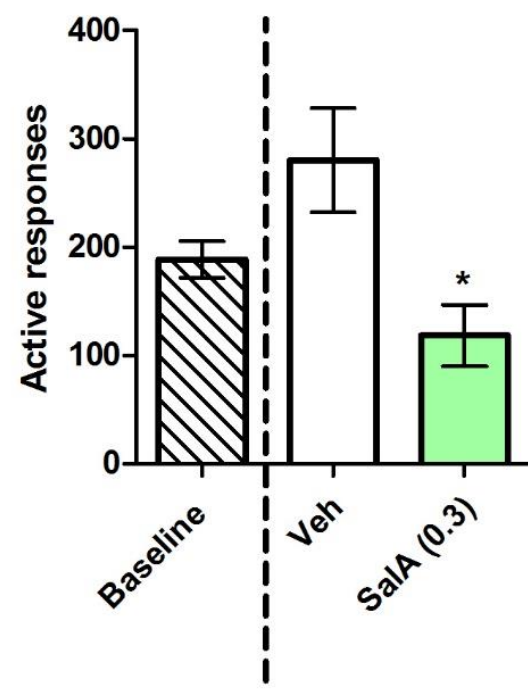

Pre-treatment

D

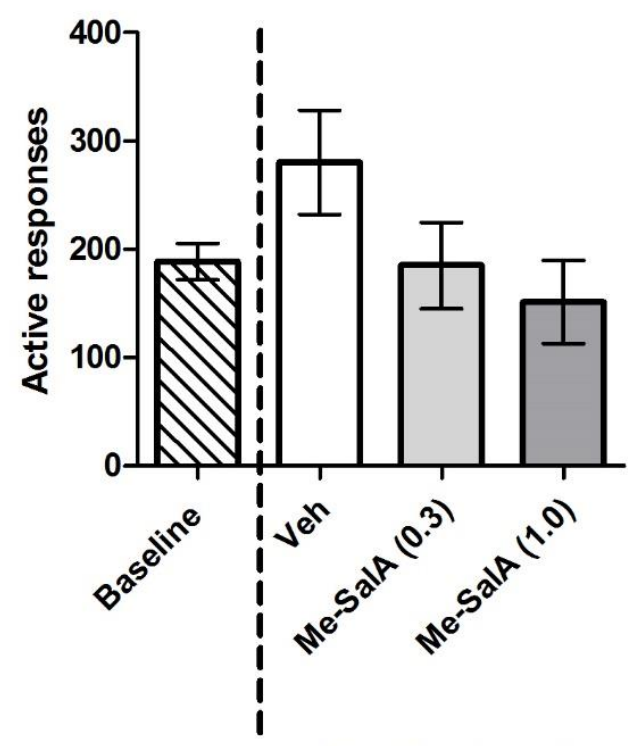

Pre-treatment

Figure 2.7. Cocaine-primed reinstatement of self-administration behaviour with $\mathrm{KOPr}$ agonist pre-treatment. Responses during the extinction phase of the reinstatement test were significantly lower than baseline responses for cocaine. Active responding for selfadministered cocaine was restored after extinction by a single priming injection of cocaine $(20 \mathrm{mg} / \mathrm{kg}$ ) without vehicle or KOPr agonist pre-treatment (A). Pre-treatment with Ethy-SalA before a priming injection of cocaine $(20 \mathrm{mg} / \mathrm{kg})$ significantly reduced active lever responses made at doses of 0.1 and $0.3 \mathrm{mg} / \mathrm{kg}$. SalA also significantly attenuated lever responses at a dose of $0.3 \mathrm{mg} / \mathrm{kg}$ (B). Pre-treatment with Me-SalA was not found to significantly attenuate active lever responses when at either $0.3 \mathrm{mg} / \mathrm{kg}$ or $1 \mathrm{mg} / \mathrm{kg}$ treatment $(\mathrm{C}) .{ }^{*}=\mathrm{p}<0.05 ;{ }^{* *}=$ $p<0.01 ;{ }^{* * *}=p<0.001$ (one-way repeated measures ANOVA, with Dunnett's multiple comparison post-test $(A, C, D)$ or repeated measures $t$-test $(B) . n=5-7$ rats. 


\subsubsection{Progressive-ratio self-administration}

Rats pre-treated with U-69,593 $(1.0 \mathrm{mg} / \mathrm{kg})$ achieved fewer infusions in a progressive-ratio session (8.44 \pm 1.27 infusions) than when treated with vehicle (12.6 \pm 0.44 infusions) $[t(8)=3.54, p<0.01]($ Fig. 2.8A). Pre-treatment with Ethy-SalA $(0.3$ and $1.0 \mathrm{mg} / \mathrm{kg})($ Fig. 2.8B) did not identify any significant differences in comparison to vehicle treatment $[F(2,10)=1.22, p>0.05]$. Similar analysis of rats pre-treated with 1.0 and $2.0 \mathrm{mg} / \mathrm{kg} \mathrm{Me}$ SalA also did not detect significant differences to vehicle treatment (Fig. 2.8C).

Rats pre-treated with higher doses of both SalA $(2.0 \mathrm{mg} / \mathrm{kg})$ and Ethy-SalA $(2.0 \mathrm{mg} / \mathrm{kg})$ and vehicle (Fig. 2.8D) showed significant differences in mean infusions achieved in comparison to vehicle $[\mathrm{F}(2,6)=3.98, p<0.05]$. Rats treated with Ethy-SalA $(2.0 \mathrm{mg} / \mathrm{kg})$ showed reduced numbers of infusions $(10.00 \pm 1.62)$ when compared to vehicle treatment (13.10 \pm 0.67 infusions) [Dunnett's multiple comparison post-test, $p<0.05$ ]. Treatment with SalA (2.0 mg/kg) had no significant effect upon mean infusions. 
A
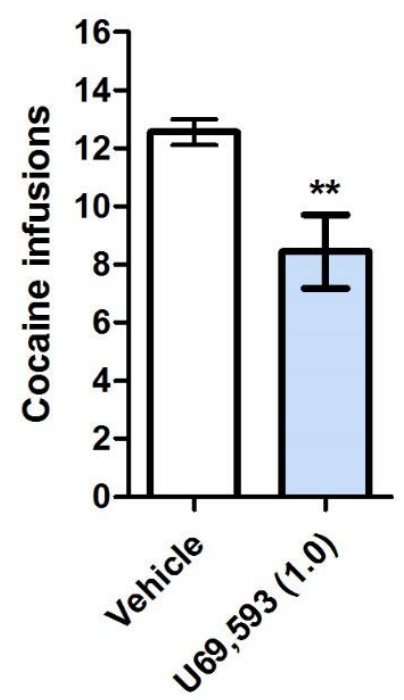

Pre-treatment

C
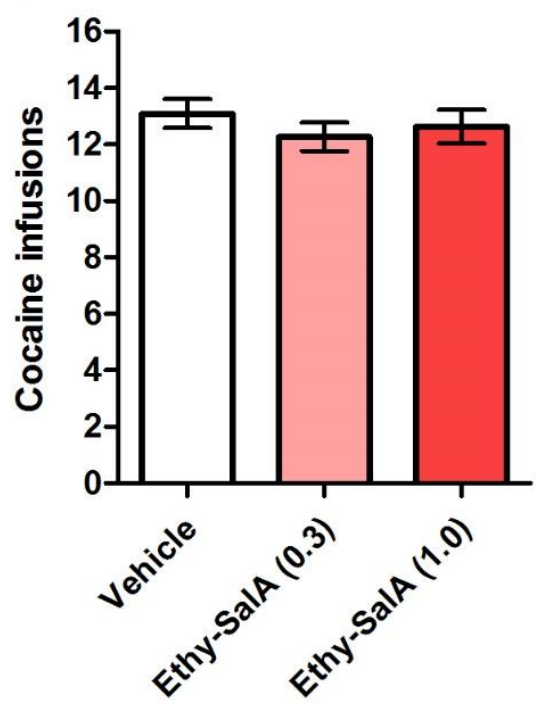

Pre-treatment
B
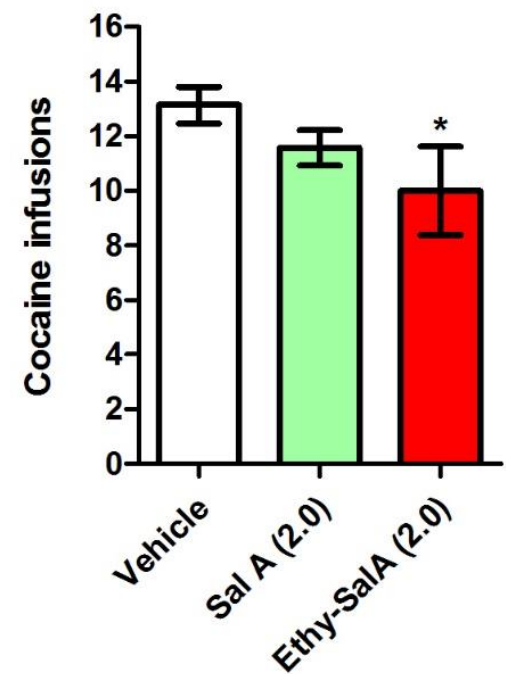

Pre-treatment

D
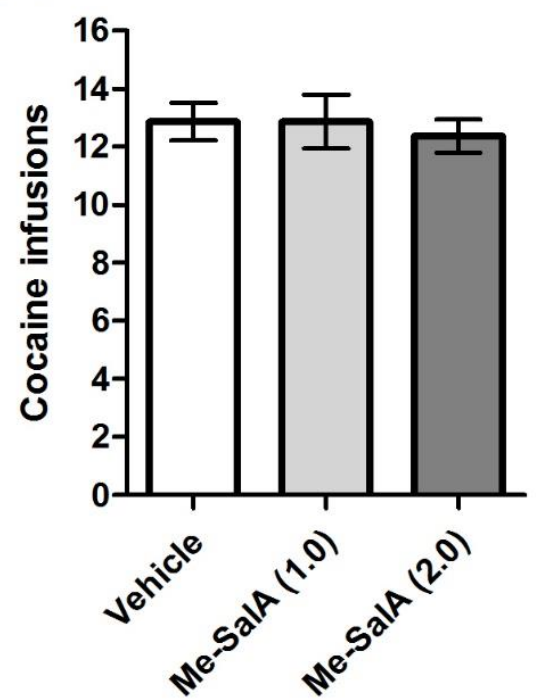

Pre-treatment

Figure 2.8. Progressive ratio cocaine self-administration with KOPr agonist pretreatment. Rats pre-treated with $1.0 \mathrm{mg} / \mathrm{kg}$ of U-69,593 achieved significantly fewer cocaine infusions per test session than rats treated with vehicle (A). Treatment with 0.3 and $1.0 \mathrm{mg} / \mathrm{kg}$ of Ethy-SalA (B) and 1.0 and $2.0 \mathrm{mg} / \mathrm{kg}$ of Me-SalA (C) had no significant effect on infusions achieved. Treatment with $2.0 \mathrm{mg} / \mathrm{kg}$ of SalA also did not cause a significant change in infusion number, but $2.0 \mathrm{mg} / \mathrm{kg}$ of Ethy-SalA significantly attenuated infusions achieved (D). ${ }^{*}=p<0.05 ;{ }^{* *}=p<0.01$ (paired t-test $(A)$ or repeated-measures oneway ANOVA with Dunnett's post-test: comparison to vehicle (B-D)). ESA= Ethy-SalA, MeSA $=$ Methy-SalA. $n=6-11$ rats. 


\subsubsection{Cocaine hyperactivity with KOPr agonist pre-treatment}

One-way ANOVA analysis of hyperactive locomotor activities with vehicle or KOPr agonist pre-treatment and cocaine or saline treatment identified significant differences between mean ambulatory responses $[F(4,45)=3.68, p<0.05]$ (Fig. 2.9A) and mean stereotypic responses $[F(4,450=2.90, p<0.0001]$ (Fig. 2.9B). Saline-primed rats treated with Ethy-SalA $(0.3 \mathrm{mg} / \mathrm{kg})(1090 \pm 239$ counts $)$ or vehicle (1029 \pm 144 counts) showed significantly fewer mean ambulatory counts than rats pre-treated with vehicle and primed with cocaine (hyperactive control) $(20 \mathrm{mg} / \mathrm{kg})(4246 \pm 886$ counts) [Dunnett's multiple comparison post-test, $p<0.05]$. A significant reduction was detected in the mean stereotypic counts of cocaine-primed rats pre-treated with U-69,593 $(0.3 \mathrm{mg} / \mathrm{kg})(5574 \pm$ 731 counts) compared to the hyperactive control (124511 \pm 810 counts) [Dunnett's multiple comparison post-test, $p<0.001$ ], and between saline-primed rats treated with Ethy-SalA (5278 \pm 566 counts) or vehicle $(5734 \pm 453)$ and the hyperactive control $[p<0.001]$.

Time course analysis of rats pre-treated with vehicle, Ethy-SalA or U-69,593 (Fig. 2.10A) indicated a significant effect of time upon ambulatory activity $[F(18,558)=13.01, p<0.001]$, but no significant differences between the treatment groups [Bonferroni post-test, $p>0.05]$. Similar analysis of stereotypic counts (Fig. 2.10B) also identified a significant interaction between time and treatment, $[F(36,558)=3.77, p<0.0001]$, with significant difference in stereotypy between U-69,593 and vehicle treated rats between 40-55 minutes and 65-85 minutes [Bonferroni post-test, $p<0.05]$. 

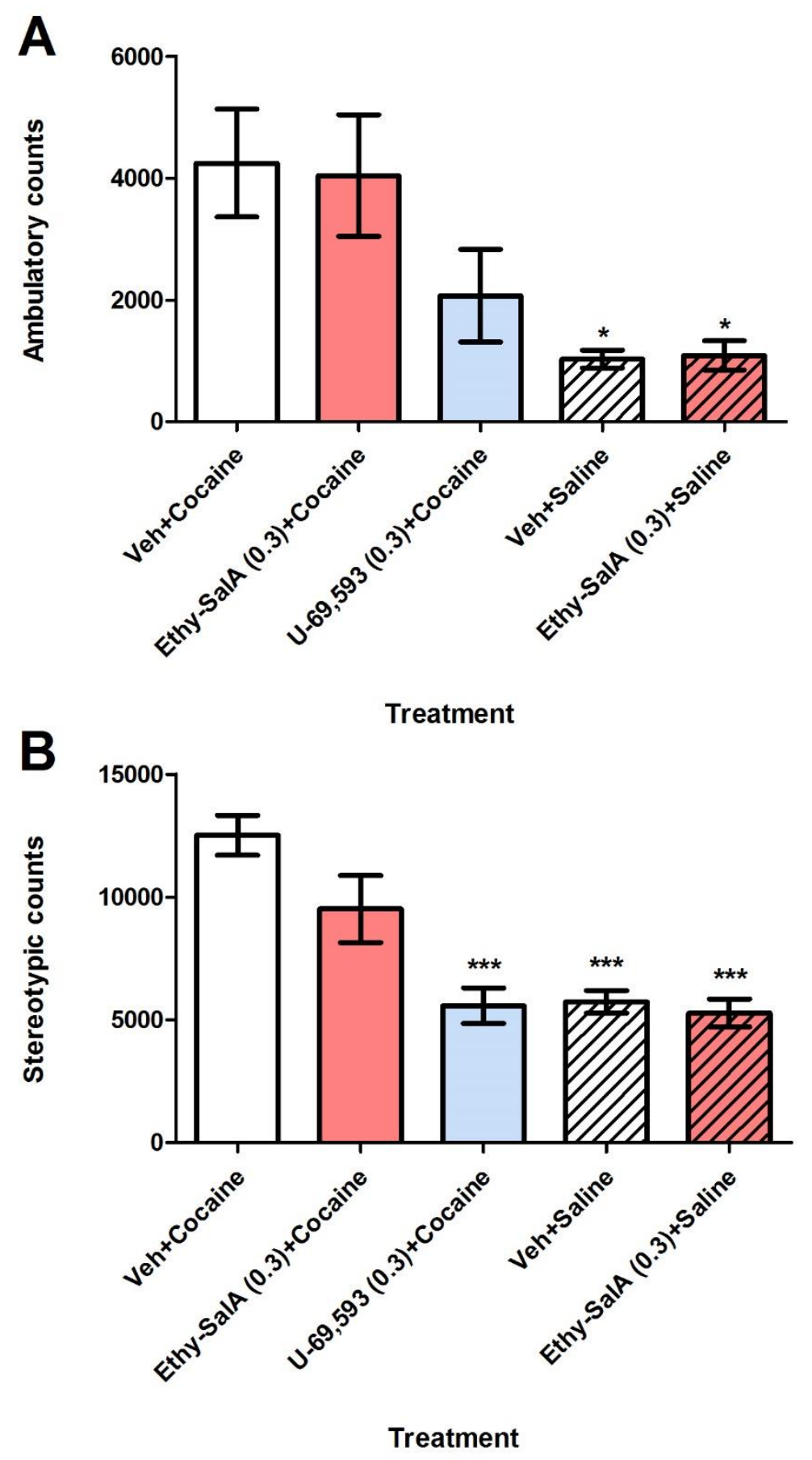

Figure 2.9. Measures of Cocaine hyperactivity with KOPr agonist pre-treatment. Rats were treated with Ethy-SalA $(0.3 \mathrm{mg} / \mathrm{kg})$ or U-69,593 $(0.3 \mathrm{mg} / \mathrm{kg}) 10$ and $15 \mathrm{~min}$ before a priming injection of cocaine $(20 \mathrm{mg} / \mathrm{kg})$ or saline, after 30 -min habituation to an activity chamber. Ethy-SalA pre-treatment did not significantly reduce ambulatory (A) or stereotypic (B) cocaine hyperactivity. U-69,593 pre-treatment significantly reduced stereotypic (B) but not ambulatory hyperactivity (A). Animals treated with vehicle or EthySalA and saline did not show a hyperactive response $(A, B)$. Veh $=$ vehicle. ${ }^{*}=p<0.05 ;{ }^{* * *}=$ $p<0.001$ (one-way ANOVA with Dunnett's post-test: comparison to Veh+Cocaine). $n=7$ 15 rats. 


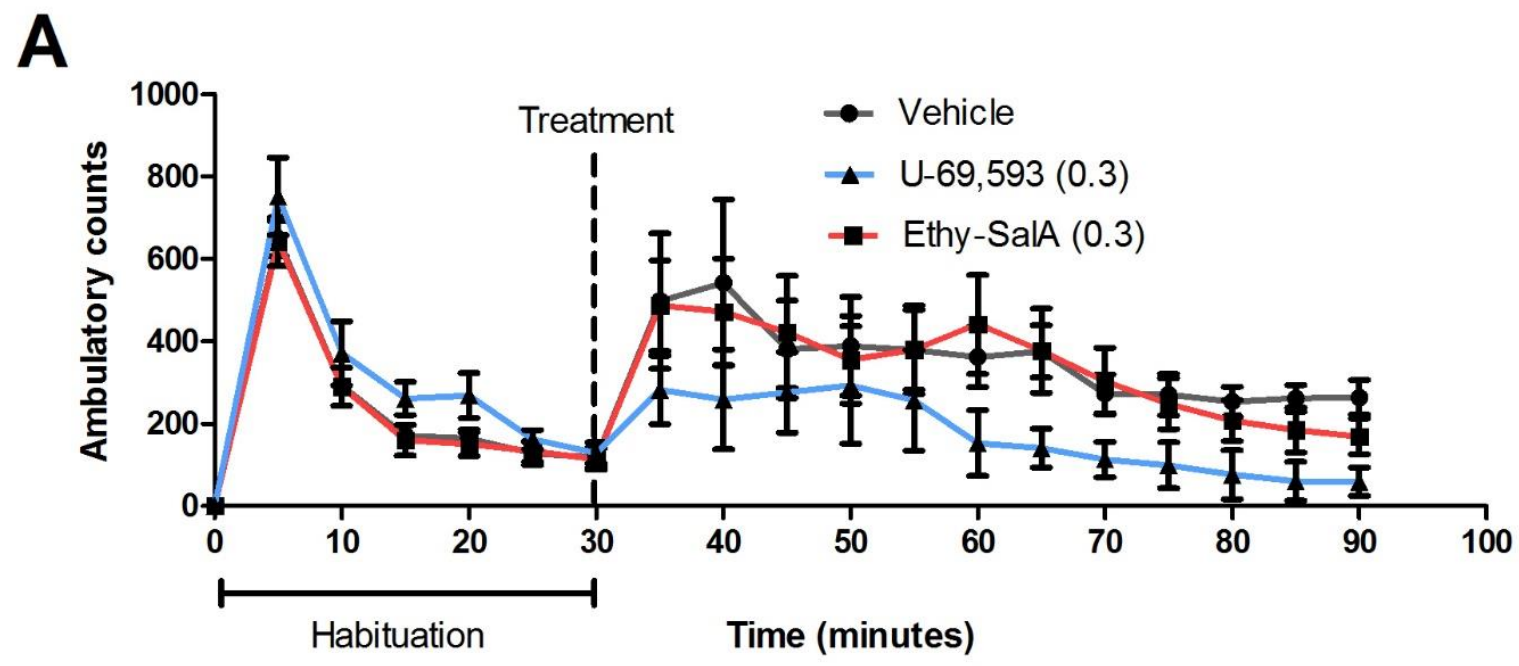

B

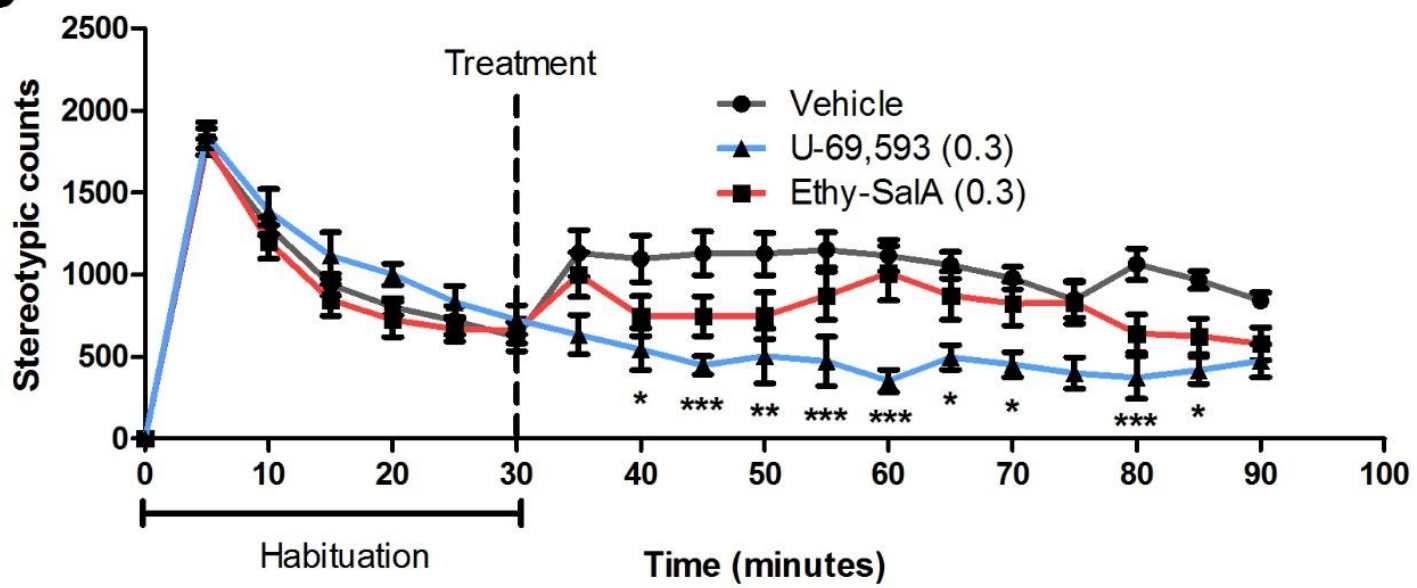

Figure 2.10. Time course analysis of cocaine hyperactivity with KOPr agonist pretreatment. Analysis of cocaine-induced ambulatory counts taken every 5 minutes (A) showed no significant differences between treatment groups. Analysis of stereotypic counts (B) indicated a reduction in stereotypic behaviour from 40-55 minutes and 65-85 minutes in rats pre-treated with U-69,593 $(0.3 \mathrm{mg} / \mathrm{kg})$ before a cocaine prime $(20 \mathrm{mg} / \mathrm{kg})$. No significant differences in activity during the habituation phase were detected between treatment groups. ${ }^{*}=p<0.05,{ }^{* *}=p<0.01,{ }^{* * *}=p<0.001$ (two-way ANOVA, with Bonferroni post-test). $n=7-15$ rats. 


\subsubsection{Behavioural sensitisation to cocaine with acute KOPr treatment}

Analysis of ambulatory responses on Day 1 vs. Day 10 indicated a significant effect of time upon mean ambulatory counts $[F(1,18)=25.81, p<0.0001]$ (Fig. 2.11A). No significant differences in ambulatory means between treatment groups were detected on either Day 1 or Day 10. Similar analysis of stereotypic responses on Day 1 vs. Day 10 identified a significant interaction between Day and treatment $[F(3,18)=5.50, p<0.01]$ (Fig. 2.11B). Bonferroni post-test analysis identified significant differences in mean stereotypic counts between the Ethy-SalA pre-treated chronic-cocaine and chronic-saline treatment groups on Day 1 of sensitisation $[p<0.01]$, and between vehicle pre-treated chronic-cocaine and chronic-saline treatment groups on Day $1[p<0.001]$. No significant differences in mean ambulatory counts were detected between control (saline) and sensitised (cocaine) treatment groups on Day 1 or 10 (Fig. 2.11B $)$. Bonferroni post-test analysis of activity counts within treatment groups on Day 1 and Day 10 (Fig. 2.11C) identified a significant increase in ambulatory counts in the chronic cocaine/Ethy-SalA group on Day 10 (20730 $\pm 3402)$ compared to Day $1(6379 \pm 2087)[p<0.01]$. Post-test analysis of stereotypic activity within treatment groups ( $\underline{\text { Fig. 2.11D) }}$ identified significant differences between Day 1 and Day 10 activity counts in groups receiving chronic cocaine with Ethy-SalA pretreatment (Day $1=14530 \pm 2336$, Day $10=20610 \pm 1024)[p<0.05]$, chronic saline with vehicle pre-treatment (Day $1=4872 \pm 709$, Day $10=19940 \pm 1113)[p<0.001]$, and chronic saline with Ethy-SalA pre-treatment (Day $1=5449 \pm 609$, Day $10=15430 \pm 2343)[p<0.01]$. 

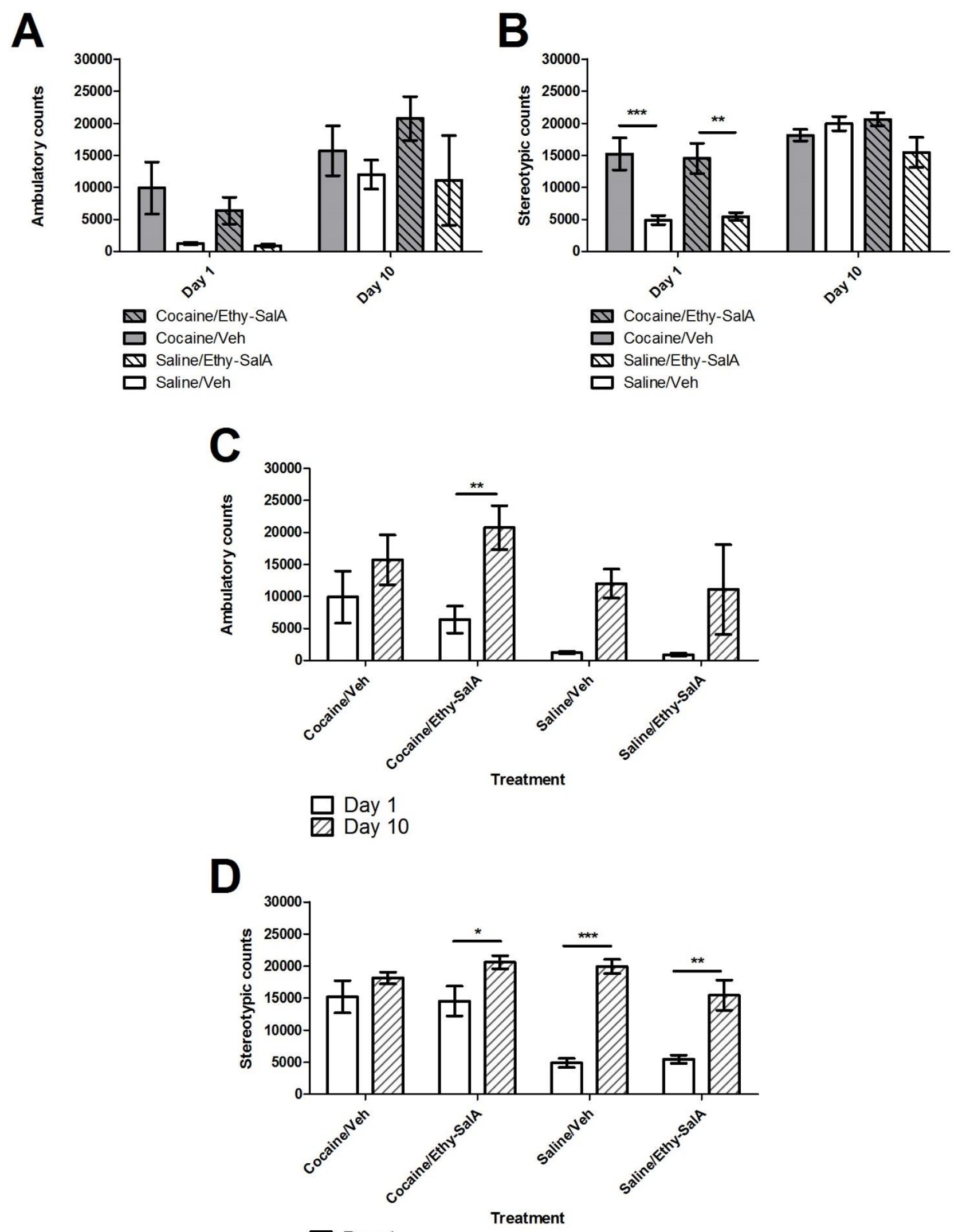

$\square$ Day 1 
Figure 2.11. Cocaine sensitisation testing with KOPr agonist pre-treatment. Comparisons of open-field locomotor activity recorded on Day 1 and Day 10 of the sensitisation experiment. Rats received only cocaine or saline on Day 1, and ESA or Veh pre-treatment followed by an injection of cocaine on Day 10. Treatment groups showed no significant difference in ambulatory behaviour during the 1-hour testing phase on Day 1 or Day 10 (A). Cocaine/Veh and Cocaine/ESA groups showed a significant increase in stereotypic behaviour on Day 1 compared to their control groups (Saline/Veh and Saline/ESA) (B). Cocaine/ESA rats showed a significant increase in ambulatory behaviour on Day 10 compared to Day 1 (C). Stereotypic behaviour was increased in Cocaine/ESA, Saline/ESA and Saline/Veh treatment groups between Day 1 and Day $10 .{ }^{*}=p<0.05$, ${ }^{* *}=p<0.01,{ }^{* * *}=p<0.001$ (repeated measures two-way ANOVA, Bonferroni post-test). Veh= vehicle. $n=5-6$ rats per treatment group. 


\subsection{Discussion}

This chapter examines the anti-cocaine properties of the novel KOPr agonists Ethy-SalA and Me-SalA in two self-administration models and two locomotor activity models in rats. The use of multiple behavioural models allows for a more complete characterisation of the effects of KOPr activation upon cocaine-associated behaviours, including drug-seeking, reward motivation, hyperactivity and sensitisation. These experiments represent the first in vivo tests of these novel compounds, and are a crucial first step in the pre-clinical identification of a novel anti-addictive pharmacotherapy.

\subsubsection{Operant cocaine conditioning}

Self-administration is a widely-used procedure in addiction research, and is generally considered to be the best way of modelling drug-taking in a laboratory setting, with intravenous self-administration using rats being the most common method. Cocaine selfadministration is widely used, due to its well-documented reinforcing effects, and the high rate of successful acquisition seen in operant training using this drug (Pickens \& Thompson, 1968). The operant training method used (Weeks, 1962) relies upon the assumption that rats will form a strong association between an active lever and delivery of the drug, and that responding on the active lever will be reinforced by the near immediate rewarding effects of intravenous cocaine (Ahmed, 2012). A strong preference for the active lever during cocaine self-administration was observed in all rats used for cocaineprimed reinstatement (Fig. 2.4B). This active vs. inactive lever discrimination was also consistently observed during FR5 baseline cocaine-administration phases (Fig. 2.5A), indicating a strong association between the active lever and drug delivery in these rats. The observation that inactive lever responses did not significantly increase during the 'extinction' saline-administration phase of the reinstatement courses (Fig. 2.5B) suggests 
that saline-administration produced true extinction of drug-seeking behaviour, and not simply transferral of the behaviour to the opposite lever.

\subsubsection{Models of drug relapse and reward motivation: cocaine-reinstatement and progressive-ratio testing.}

Cocaine-primed reinstatement and progressive-ratio self-administration tests were used to evaluate two separate addiction-associated behaviours. The reinstatement model has been historically used to test ability of drug-cues, environmental cues or stressors to reinstate drug-seeking behaviour (Epstein et al., 2006), while the progressive-ratio model tests the motivational potential of an administered drug or other reinforcer (Richardson \& Roberts, 1996). These two models approximate two different phases of the addictive cycle proposed by Koob et al. (see Chapter 1, Fig.1.2): relapse to drug-seeking/craving, and active drug taking behaviour. A reduction in the number of active responses evoked by a reinstating cocaine prime, or a reduction in the total number of infusions achieved on a progressive-ratio schedule can indicate that a pre-treatment has interfered with the reinforcing, rewarding effects of cocaine, or that the pre-treatment has reduced the rat's motivation to continue drug-seeking.

In order to detect the ability of cocaine to re-awaken drug-seeking in rats, the cocainereinstatement model requires that baseline drug-seeking behaviour is extinguished before reinstatement (Yeo et al., 2003). Matching previous observations by Davis and Smith (1976), extinction of active lever responding was achieved by replacing the administered drug with $0.9 \%$ saline solution, with the lack of a rewarding stimulus perturbing the rat's association of the active lever with reward (Fig. 2.6A). Rats receiving $0.3 \mathrm{mg} / \mathrm{kg}$ of SalA showed reduced mean reinstatement responses in comparison to vehicle, an effect replicated from an earlier study of the behavioural effects of SalA (Morani et al., 2009). 
Ethy-SalA is shown here to attenuate cocaine reinstatement in rats at a lower dose than SalA (0.1 mg/kg compared to $0.3 \mathrm{mg} / \mathrm{kg}$ ) (Fig. 2.6C). The compound Me-SalA does not show comparable potency and has a more variable effect (ig. 2.6D), and may only be an effective inhibitor of cocaine reinstatement at dosages higher than those tested in this experiment $(0.3$ and $1.0 \mathrm{mg} / \mathrm{kg})$.

The progressive-ratio self-administration paradigm was used in this thesis to explore the effect of acute doses of novel and existing KOPr agonists upon the reinforcing effects of cocaine, and the ability of KOPr agonist treatment to alter the motivational qualities of cocaine. Progressive-ratio has been previously used to examine differences in response in animals previously sensitised to an abusive drug (Mendrek et al., 1998), comparisons of reinforcement between different drugs (Roberts, 1993), and the effect of a pre-treatment upon the reinforcing effects of a drug of abuse (McGregor et al., 1993), which this study uses. Changes to a 'break-point', or the point at which an animal will cease to attempt to self-administer the drug on offer, are used to gauge the subject's relative level of motivation (Stafford et al., 1998).

The results of the cocaine-primed reinstatement testing for Ethy-SalA and Me-SalA has been published along with another novel KOPr agonist, 16-bromo Salvinorin A (Br-SalA), which has also been shown to significantly reduce reinstatement responses at $1.0 \mathrm{mg} / \mathrm{kg}$ (Riley et al., 2014). Cellular assays accompanying the behavioural data show Ethy-SalA to be a more potent activator of the KOPr than SalA and Br-SalA in a cellular model of KOPr-mediated CAMP-inhibition, while Me-SalA shows an almost 14-fold reduction in potency compared to SalA (see Table 2.2). To date, Ethy-SalA is the most potent KOPr agonist generated from carbon-16 modifications to the structure of SalA. As well as attenuating cocaine-primed reinstatement at a lower dose than other tested compounds, Ethy-SalA is the only SalA-derived KOPr agonist shown to effectively attenuate progressive-ratio, at dose of $2.0 \mathrm{mg} / \mathrm{kg}$. The enhanced cellular activity displayed by EthySalA may explain its increased potency in comparison to SalA in cocaine-reinstatement 
tests, and the ability to attenuate progressive-ratio self-administration. Reduced potency at the KOPr could explain the more variable effect seen with Me-SalA pre-treatment, and the lack of significant effect detected in progressive-ratio. The increased potency of EthySalA may also be contributing to a sedative effect, particularly at the relatively high dose of $2 \mathrm{mg} / \mathrm{kg}$, preventing the test rat from physically performing the operant task. Unpublished data from tests performed by another student of the lab indicates that EthySalA does not show significant sedative effects at $2 \mathrm{mg} / \mathrm{kg}$ in cocaine-naïve rats. Further tests of baseline locomotor activity or motor coordination through motion tracking and rotarod assays would help confirm this observation.

The anti-cocaine effect observed in this behavioural model is most likely due to a suppression of DA signalling caused by KOPr activation within the reward pathway, which has been observed and confirmed in rat models both in vivo (Mueller et al., 2003) and ex vivo (Cortez et al., 2010; Dasgupta et al., 2003). By activating the KOPr system prior to an injection of cocaine, the rewarding stimulus of the cocaine is theorised to be blunted, reducing the efficacy of cocaine to reinstate drug-taking behaviour. The ability of KOPr agonists to attenuate cocaine-primed reinstatement has been confirmed with a number of classical KOPr agonists and novel SalA derivatives (Morani et al., 2013 ; Morani et al., 2009 ; Prevatt-Smith et al., 2011; Simonson et al., 2015 ), and appears to be a characteristic effect of KOPr activation in the context of cocaine priming. The ability of $\mathrm{KOPr}$ agonism to alter progressive-ratio responding is less well studied. A dose of 2.0 $\mathrm{mg} / \mathrm{kg}$ SalA has shown the ability to attenuate progressive-ratio sucrose responding in rats (Ebner et al., 2010), and recent testing has indicated that co-infusions of SalA with oxycodone can reduce progressive-ratio break-points in rhesus monkeys (Naylor et al., 2015). The experiments performed by A. Ewald (PhD thesis, 2015) and the work of this thesis represent the first tests of acute KOPr agonist treatment in a progressive-ratio model of self-administration. 


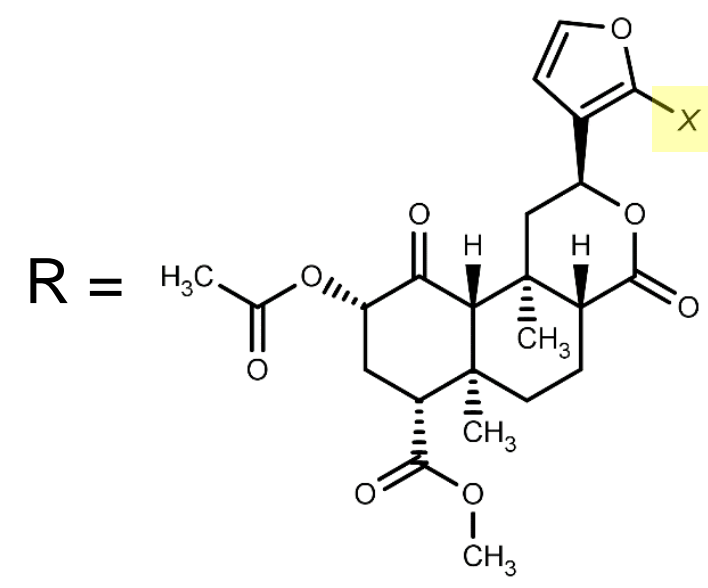

\begin{tabular}{|c|c|c|c|c|}
\hline STRUCTURE & NAME & $\begin{array}{l}\mathrm{EC}_{50} \text { (cellular } \\
\text { KOPr assay*) }\end{array}$ & $\begin{array}{c}\text { MINIMUM } \\
\text { EFFECTIVE DOSE } \\
\text { (COCAINE- } \\
\text { REINSTATEMENT) }\end{array}$ & REFERENCES \\
\hline $\mathrm{R}$ & Salvinorin $A$ & $0.030 \pm 0.004 \mathrm{nM}^{(1)}$ & $0.3 \mathrm{mg} / \mathrm{kg}{ }^{(2)}$ & $\begin{array}{l}{ }^{1} \text { Riley et al., } 2014 ; \\
{ }^{2} \text { Morani et al., } 2009\end{array}$ \\
\hline & $\begin{array}{l}\text { 16-bromo- } \\
\text { Salvinorin A }\end{array}$ & $0.038 \pm 0.010 \mathrm{nM}^{(1)}$ & $1 \mathrm{mg} / \mathrm{kg}^{(1)}$ & (1) Riley et al., 2014 \\
\hline$\sim_{3}$ & $\begin{array}{l}\text { 16-methyl- } \\
\text { Salvinorin A }\end{array}$ & $0.407 \pm 0.147 \mathrm{nM}^{(1)}$ & Not observed & (1) Riley et al., 2014. \\
\hline & $\begin{array}{l}\text { 16-ethynyl- } \\
\text { Salvinorin A }\end{array}$ & $0.019 \pm 0.004 \mathrm{nM}^{(1)}$ & $0.1 \mathrm{mg} / \mathrm{kg}{ }^{(1)}$ & (1) Riley et al., 2014. \\
\hline
\end{tabular}

Table 2.2. Carbon-16 analogues of SalA. Chemical structures and comparative potencies $\left(E C_{50}\right)$ of Salvinorin $A$ and analogues arising from cycloaddition to the carbon16 position. All effective doses are i.p. injections of compound in rats. *Assays for potency at the KOPr conducted by measuring inhibition of forskolin-induced cAMP accumulation. Assays were performed in $\mathrm{CHO}$ cells stably expressing $\mathrm{KOPr}$ (unpublished data, Prisinzano lab). Cocaine-reinstatement effective doses for Ethy-SalA and Me-SalA represent findings from this thesis published in 2014. 


\subsubsection{Models of cocaine locomotor effects: cocaine hyperactivity and behavioural sensitisation}

Treatment with the effective cocaine-reinstatement attenuating dose of Ethy-SalA (0.3 $\mathrm{mg} / \mathrm{kg}$ ) was not found to significantly affect total ambulatory or stereotypic count in either acute cocaine hyperactivity (Fig. 2.9) or sensitised hyperactivity (Fig. 2.11) assays of openfield locomotion. This result is unusual, as dopaminergic activity in the mesolimbic arm of the reward pathway is closely tied to the hyperactive effects of cocaine in rats and mice (Herrmann et al., 2003; Xu et al., 1994). Therefore, if the attenuation of cocaine reward observed in cocaine-reinstatement and progressive-ratio (PR) behavioural tests were due to dopaminergic inhibition, a decrease in cocaine-induced hyperactivity would also be expected.

SalA has also shown unusual experimental results in cocaine hyperactivity tests. A high dose of SalA $(2.0 \mathrm{mg} / \mathrm{kg})$ attenuates cocaine hyperactivity in rats with a $10 \mathrm{mg} / \mathrm{kg}$ cocaine prime, but does not significantly alter cocaine hyperactivity with acute $(0.3 \mathrm{mg} / \mathrm{kg})$ (Morani et al., 2012) or chronic pre-treatment (1.0-3.2 mg/kg) (Gehrke et al., 2008) before a 20 $\mathrm{mg} / \mathrm{kg}$ cocaine challenge. Pre-treatment with the classical KOPr agonists U-69,593 $(0.3$ $\mathrm{mg} / \mathrm{kg})$ and $\mathrm{U}-50,488(5 \mathrm{mg} / \mathrm{kg})$ paired with 15 and $20 \mathrm{mg} / \mathrm{kg}$ cocaine challenges respectively has been shown to supress hyperactivity (Crawford et al., 1995; Vanderschuren et al., 2000). Other KOPr agonists derived from carbon-2 modifications have also shown the ability to attenuate cocaine hyperactive behaviour with a $20 \mathrm{mg} / \mathrm{kg}$ cocaine challenge (unpublished data, Kivell laboratory). U-69,593, which was used as a positive control for cocaine hyperactivity testing (as shown by Vanderschuren et al., 2000), was found to attenuate stereotypic behaviour but not total ambulatory counts. The lack of observed effect may be due to differences in tracking software (EthnoVision as opposed to Med Associates tracking software) and the parameters for behavioural scoring, as well as differences in rat strain (Wistar as opposed to Sprague-Dawley) and the dose of cocaine 
used (15 mg/kg in comparison to $20 \mathrm{mg} / \mathrm{kg})$. A high dose of SalA (2.0 mg/kg) was initially intended as the positive control based upon an experiment by Chartoff et al. (2008) using a $10 \mathrm{mg} / \mathrm{kg}$ cocaine challenge, but preliminary tests indicated that SalA treatment was enhanced cocaine hyperactivity (Appendix 5, Fig 6.4).

The atypical effects of SalA observed in this thesis and in other literature, and that of EthySalA, may be due to the interference of hallucinogenic and off-target dopaminergic effects. Acute pre-treatment with the classic serotonergic hallucinogens LSD and N,Ndimethyltryptamine (DMT) has previously been shown to enhance the dopaminergic hyperactivity caused by drugs such as amphetamine and apomorphine (Fink et al., 1979), suggesting that the interaction of 5-HT and DA pathways may have an important role in regulating hyperactivity. SalA does not interact directly with $5-\mathrm{HT}$ receptors, and discriminates from the effects of LSD and other serotonergic hallucinogens in both primate and rodent behavioural tests (Butelman et al., 2010; Killinger et al., 2010). However, SalA has been shown to inhibit SERT, and therefore very likely influences $5-\mathrm{HT}$ levels within the brain (Kivell et al., 2014). Unusually, SalA has also been shown to stimulate $D_{2}$ receptors in ex vivo rat striata, an effect which was blocked by $D_{2}$ antagonism (Seeman et al., 2009). D2 and D1-receptor co-activation influences expression of cocaine-induced locomotor behaviours in rats (Arnt et al., 1988; Ushijima et al., 1995), and $D_{2}$ agonism or indirect activation by SalA and potentially Ethy-SalA could explain the failure of these compounds to significantly attenuate acute cocaine hyperactivity at low doses.

Behavioural sensitisation in rats was used in an attempt to establish whether the anticocaine effects of Ethy-SalA were dependent upon prior chronic experience to cocaine. However, the control rats used for this study did not develop significant locomotor sensitisation to the effects of cocaine after five consecutive days of high-dose cocaine (20 $\mathrm{mg} / \mathrm{kg}$ ), limiting any meaningful comparison to Ethy-SalA treatment. Cocaine sensitisation is a robust procedure which has been successfully used in conjunction with KOPr agonist treatment to establish the effects of chronic and acute KOPr activation upon sensitised 
cocaine hyperactivity (Heidbreder et al., 1995; Heidbreder \& Shippenberg, 1994 ; Morani et al., 2009 ). The lack of cocaine sensitisation detected in this experiment may be due to high variability in hyperactive responses coupled with relatively low numbers (5-6 rats per treatment group). The animals used for this experiment were also taken from another facility (the School of Psychology) and, despite a week's rest before the start of experimentation, may have been suffering from move-related stress, which likely would have influenced behavioural results.

\subsubsection{Limitations and future directions}

The self-administration model is one of the standard models used in addiction research, but does have limitations in its ability to completely and accurately model human addiction. It has been observed that only a relatively small sub-set of animals regularly exposed to drugs of abuse will develop true 'addiction' or uncontrollable craving for drug (Ahmed, 2005), particularly in rats allowed only restricted access to drug (1-2 hours) as opposed to extended access of 6 hours or greater (Ahmed \& Koob, 1998). As rats for both cocainereinstatement and progressive-ratio paradigms were trained using a restricted access model, it is arguable whether the animals used in this study could be considered 'dependant' upon cocaine and to have developed the strong association necessary to accurately model drug addiction in humans. The reward salience of cocaine in rats may also not be as great as it is in humans. It is significant that the majority of rats (an estimated $94 \%$ of an experimental population) will select a saccharin reward over a cocaine infusion when given the choice, regardless of the dosage of drug on offer (Lenoir et al., 2007). The development of operant cocaine acquisition can even be attenuated if rats are exposed to other reinforcers such as food or glucose/saccharin solutions (Campbell \& Carroll, 2000).

Rats trained under extended access conditions would be much harder to extinguish behaviour in, and would presumably respond much more strongly to a cocaine prime, 
making attenuation of drug-seeking behaviour much harder to achieve. The development of addiction in humans also involves a number of social factors and cues (Quigley \& Collins, 1999) which cannot be modelled well in a laboratory situation, and which the selfadministration paradigm does not account for, though there is evidence that the social isolation which self-administration rats are placed under after surgery may enhance responsiveness to drugs of abuse such as cocaine and amphetamine (Jones et al., 1990; Schenk et al., 1987).

The Latin square treatment designs used for progressive-ratio and cocaine-primed reinstatement testing, rats received multiple injections of KOPr agonist over time, as part of a within-subject treatment design. This design assumes that single acute treatments of KOPr agonists do not have long-lasting effects upon the brain that may interfere with subsequent behavioural testing. A study by Zhang et al. (2005) showed that a single injection of SalA at $3.2 \mathrm{mg} / \mathrm{kg}$ reduced DA levels in the mouse caudate putamen for a period of 10 hours, but that after 23 hours this effect had dissipated, with DA levels returning to baseline (Zhang et al., 2005). A recent study also showed that a high dose of SalA $(2.0 \mathrm{mg} / \mathrm{kg})$ significantly affected the reward potential of cocaine for up to 24 hours after acute injection in rats (Chartoff et al., 2015), despite the fact that SalA is almost entirely metabolised and cleared from the brain 60 minutes after i.p. injection (Hooker et al., 2009). To allow for complete clearance of Me-SalA and Ethy-SalA, and for behavioural effects to subside, rats were allowed a rest period of 48 hours before resuming cocaine administration. Rats in this study received acute KOPr agonist treatments at least seven days apart, whereas significant behavioural effects for multiple KOPr treatments have only been detected with daily injections of KOPr agonists (Chartoff et al., 2008; Heidbreder et al., 1993; Potter et al., 2011).

The measurement and comparison of 'baseline' cocaine responding prior to each reinstatement is a useful identifier of long-term KOPr agonist effects. Chronic KOP administration could be expected to alter baseline responding for cocaine, as chronic 
exposure to SalA has been shown to increase intra-cranial self-stimulation baselines in rats (Potter et al., 2011), and conversely chronic blockade of the KOPr in rats can prevent escalation of dose in cocaine-dependant animals (Wee et al., 2009). Analysis of the mean total dose of cocaine administered during each baseline session, normalised to weight, showed no significant increase or decrease in cocaine dosage over time, suggesting that cocaine administration remained stable in these rats, even with multiple staggered injection of KOPr agonist.

Chronic cocaine administration in itself has a considerable effect upon the KOP system. Daily cocaine administration for extended periods of time (7-14 consecutive days) has been shown to increase KOPr expression in the caudate putamen and NAc of rats (Unterwald et al., 1994), an effect which enhances binding of the KOPr agonist U-69,593 (Collins et al., 2002). Chronic cocaine exposure is therefore likely to enhance KOP effects within the brain compared to drug-naïve animals. All rats used in this study for selfadministration were given a prolonged period of self-administration training, involving chronic exposure to cocaine for a minimum of 25 days. Though not tested, it could be reasonably assumed that all rats displayed this heightened KOP sensitivity prior to testing. This effect has also been observed in human subjects with a history of chronic cocaine abuse (Hurd \& Herkenham, 1993). Self-administration rats could therefore be expected to show much greater sensitivity to KOPr treatment than rats receiving a single cocaine challenge, or even multiple cocaine treatments for only 5 days, as was performed for cocaine hyperactivity and behavioural sensitisation testing.

Measures of cocaine-induced locomotor hyperactivity have been used as an alternative screen for anti-cocaine effects, or more generally for anti-dopaminergic effects. However, the results of obtained from self-administration testing in this thesis suggest that cocaine hyperactivity is not a good predictor of anti-cocaine reward effects, and that DA-dependant reward and hyperactivity processes may be regulated in significantly different ways. The NAc is a key dopaminergic area with great influence on locomotor and hyperactive 
behaviour (Delfs et al., 1990). However, significant behavioural differences have been detected between dopaminergic inhibition of the 'shell' and 'core' regions of the NAc, with inhibition of the core enhancing the development of sucrose CPP in rats, while shell DA inhibition produced the opposite effect (Ito \& Hayen, 2011). For locomotor behaviour, glutamatergic extensions into the NAc core have been shown to regulate the cocainestimulated hyperactivity in rats, but do not influence this behaviour in the shell (Pulvirenti et al., 1994). These opposing effects within the NAc are also seen with regard to kappaopioid activity: a recent optogenetic study in mice has shown that selective activation of KOPr receptors in only the dorsal or ventral regions of the NAc shell can induce entirely opposite behavioural effects, with animals showing either reward or aversion responses respectively (Al-Hasani et al., 2015). The complex sub-structural differences in behavioural output suggest that even though the NAc is a key component of both locomotor and reward pathways, the results of reward-based testing may not be predictive of locomotor behaviour in animal models, and vice versa.

Future studies of the anti-drug properties of Ethy-SalA could include more robust models of drug addiction, such extended-access self-administration, and other drugs of abuse such as the psychostimulants methamphetamine and amphetamine. Previous studies have shown that treatment with $U-69,593 \quad(0.16,0.32 \mathrm{mg} / \mathrm{kg})$ can reduce methamphetamine locomotor behaviour (Tzaferis \& McGinty, 2001), in a similar manner to the attenuation of cocaine hyperactivity. To date, SalA and its novel derivatives have not been tested in models of drug-seeking other than cocaine self-administration. The inclusion of other drug models, including opioids such as morphine and heroin, could help determine whether the potential anti-addictive effects observed for Ethy-SalA have wider applications beyond an anti-cocaine mechanism. 


\subsubsection{Conclusions}

Acute treatments of Ethy-SalA were shown to successfully attenuate cocaine-primed reinstatement $(0.1$ and $0.3 \mathrm{mg} / \mathrm{kg})$ and progressive ratio self-administration $(2.0 \mathrm{mg} / \mathrm{kg})$, but did not significantly alter cocaine-induced hyperactive behaviour or the expression of cocaine behavioural sensititsation with respect to locomotion in Sprague-Dawley rats. MeSalA was not found to significantly affect either cocaine-reinstatement or progressive ratio self-administration at the doses tested $(0.3-2.0 \mathrm{mg} / \mathrm{kg})$. 


\section{Chapter 3 : Behavioural side-effect testing of novel KOPr agonists}

\subsection{Methods}

\subsubsection{Sucrose self-administration training}

Sucrose reinforcement training was performed in the same operant chambers used for cocaine self-administration (see Chapter 2, section 2.1.4), but with a sucrose pellet delivery apparatus (Med Associates, ENV-203M-45) inset between the levers in place of an infusion apparatus. Depression of the active lever resulted in delivery of a single pellet (Dustless Precision Pellet, 45 mg sucrose: Able Scientific, Perth, AUS) and activation of the paired light for 12 seconds; depression of the left lever resulted in no outcome. Rats were placed in the operant box and allowed to self-administer sucrose pellets on an FR-1 schedule for 45 minutes. Once rats were responding more than 20 times on the active lever over three consecutive days, and with an active-to-inactive lever ratio of greater than $2: 1$, rats were placed on an FR-5 schedule. Rats were moved to the testing phase of administration when less than $20 \%$ variation in responding was observed over three consecutive days. Sucrose self-administration was run as daily 45 -minute sessions during the training phase. All responses and sucrose deliveries were recorded and controlled using Med Associates software (MED-PC IV, version 4.2).

\subsubsection{Sucrose administration with KOPr agonist pre-treatment}

Rats were injected with either U-50,488 (10 mg/kg, i.p.), U-69,593 (0.3 mg/kg, s.c.), MeSalA (0.3 mg/kg, i.p.), Ethy-SalA (0.3 mg/kg, i.p.) or vehicle 10 minutes (U-50,488, MeSalA, Ethy-SalA) or 15 minutes $(U-69,593)$ prior to the start of an administration session. A lower dose of $\mathrm{U}-50,488$ compared to the previously reported cocaine-attenuating 
effective dose of $30 \mathrm{mg} / \mathrm{kg}$ (Morani et al., 2009) was used due to the observation that a 30 $\mathrm{mg} / \mathrm{kg}$ dose caused complete sedation in an initial test. Rats were placed into operant chambers set up as described for sucrose administration training and allowed to selfadminister sucrose for 45 minute, with responses being recorded. Treatment order was varied using a Latin square design (see Appendix, Table 6.3). All training and testing was carried out between 1300 and 1500 hours. After each test day, rats were given two days of rest to allow the KOPr agonist to pass out of the rat.

\subsubsection{Sedation testing: locomotor activity}

Measures of locomotor activity were used in this study to identify potential sedative affects caused by the novel KOPr agonists, at the doses used for cocaine-reinstatement testing. Rats weighing between 280-400g were placed in activity chambers (ENV-520, Med Associates) within light- and sound-attenuating boxes for a 30-minute habituation period prior to administration of vehicle, Me-SalA (1 mg/kg, i.p.) or Ethy-SalA (0.3 mg/kg, i.p.). Rats were then placed back into the activity chamber and allowed to explore the space for a further 60 minutes. Stereotypic and ambulatory activity was recorded during habituation and testing sessions as previously described (see Chapter 2, section 2.1.7).

\subsubsection{Pro-depressive screening: Forced Swim Test}

The FST was used to determine the potential pro-depressive effects of the novel KOPr agonist Ethy-SalA. A modified version of the original Forced Swim Test (FST) created by Porsolt et.al (1977) was used (Slattery \& Cryan, 2012), with a habituation stage 24 hours prior to testing. On the habituation day a $40 \mathrm{~cm}$ tall cylinder of $19 \mathrm{~cm}$ diameter, closed at one end, was filled with tap water $\left(23-25^{\circ} \mathrm{C}\right)$ to a height of $30 \mathrm{~cm}$ from the bottom of the cylinder. Rats were then placed into the water-filled cylinder for a 15-minute period, after 
which the rat was removed, towelled dry and briefly blow-dried. After 24 hours, rats were pre-treated with vehicle (i.p.), Ethy-SalA (0.3 mg/kg, i.p.), or SalA (0.3 mg/kg, i.p.) 10 minutes (vehicle, Ethy-SalA) or 5 minutes (SalA) prior to being placed into the water-filled cylinder. Activity within the cylinder was filmed from a side-view for 5 minutes. Rats were then removed and towelled dry. Any rat which climbed out of the cylinder during either habituation or testing stages was excluded from the experiment. For analysis, videos were edited into 5-second blocks, and scored as either displaying either climbing, swimming or immobility for the majority of the 5 seconds. Videos were scored by a researcher blinded to the treatment groups. Following the recommendations of Slattery \& Cryan (2012), prior to experimental scoring three different videos were scored multiple times by the same researcher until the score variation was less than $10 \%$, to ensure intra-scorer accuracy.

\subsubsection{Statistical analysis}

All statistical analysis was performed using GraphPad Prism 5.0 software. One-Way ANOVA tests were used to analyse the variances of 3 or more means, and t-tests were used to analyse 2 means. Two-way ANOVA analysis was used for examining the time course effect of treatments (open-field locomotion, cocaine hyperactivity). Repeatedmeasures analysis was used for data where rats received multiple treatments (sucrose administration) or were scored for multiple behaviours (FST). Post-tests were either Bonferroni or Dunnett's multiple comparison. Results were considered to show significance if the $p$-value for statistical tests was $<0.05$. Numerical results are reported intext as Mean \pm Standard Error of Mean. 


\subsection{Results}

\subsubsection{Effects of acute KOPr agonist treatment upon sucrose self- administration}

The use of the non-drug reward stimulus sucrose was used to determine if acute administration of Ethy-SalA or Me-SalA affected natural reward in rats, at doses used in cocaine-reinstatement testing. Significant differences in mean sucrose responses were detected between treatment groups $[F(5,6)=1.380, p<0.0001]$. Rats treated with $U-50,488$ $(10 \mathrm{mg} / \mathrm{kg})$ showed a significant reduction in active responses $(150.7 \pm 59.13)$ when compared to vehicle pre-treatment $(632.9 \pm 42.2)$ [Dunnett's multiple comparison to vehicle post-test, $p<0.001]$. U-69,593, Ethy-SalA and Me-SalA pre-treatments (all 0.3 $\mathrm{mg} / \mathrm{kg}$ ) did not show a significant attenuation of sucrose administration compared to vehicle. Vehicle responses were also not significantly different to mean baseline responses for sucrose (Fig. 3.1). Analysis of mean active and inactive self-administration responses during five 3-day baseline phases between testing days was used to test the assumptions that (1) the active lever would be favoured over the inactive lever and (2) that the amount of sucrose administered in each successive baseline phase would not alter significantly. A significant interaction between session number (time) and the numbers of active and inactive responses was detected $[F(4,30)=4.20, p<0.001]$ (Fig. 3.2A). The numbers of mean active responses were significantly greater than inactive responses for each baseline session [Bonferroni post-test, $p<0.001]$. Linear regression analysis of the mean dose of sucrose (grams) self-administered by rats in successive 3-day baseline sessions (Fig. 3.2B) showed a weak positive correlation between time and sucrose dose [Pearson correlation coefficient, $\left.\mathrm{r}^{2}=0.2854\right]$. The slope of the linear model of dose against time was significantly non-zero $[F(1,33)=13.18, p<0.001]$. 


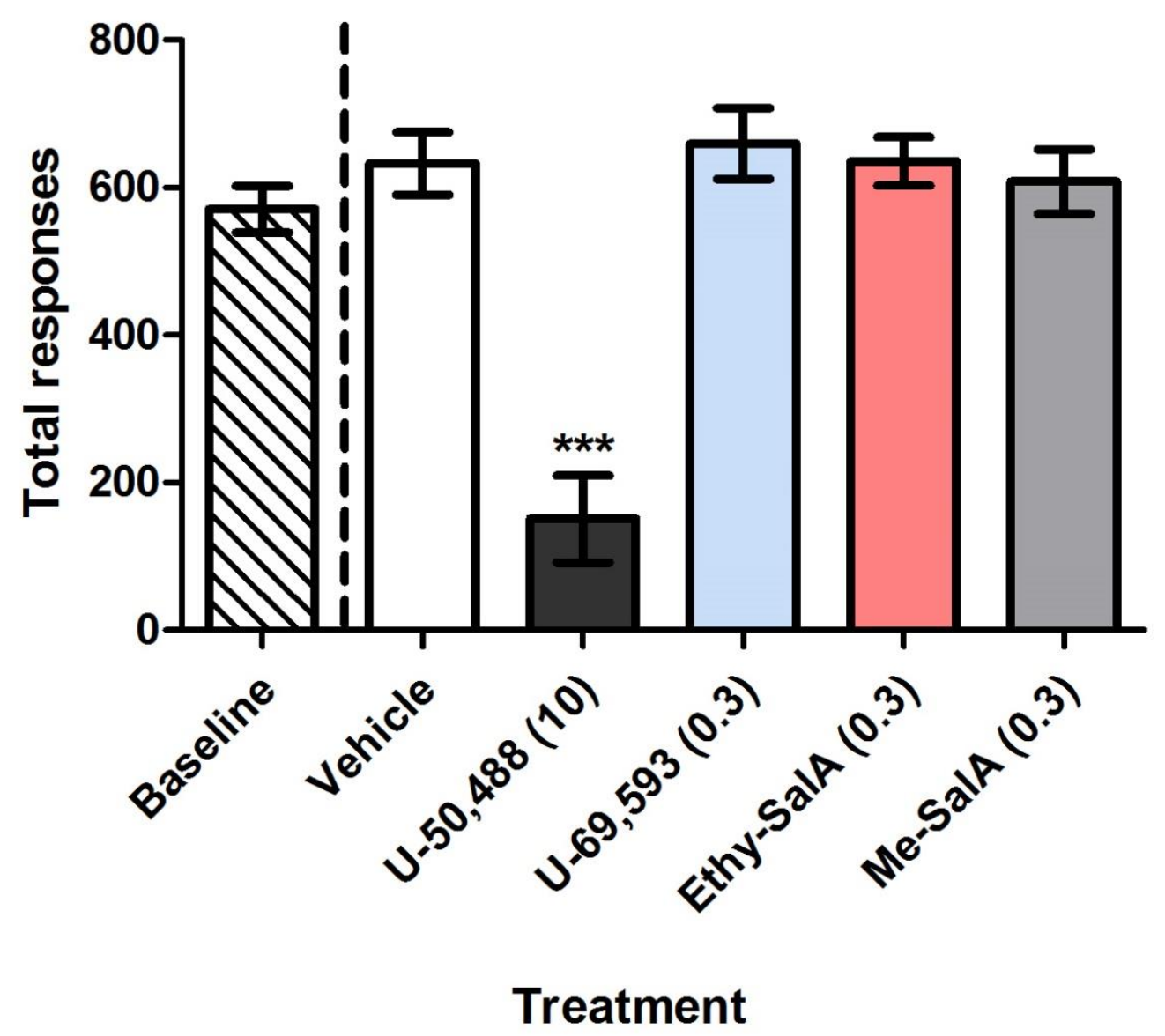

Figure 3.1. Effects of acute KOPr agonist treatment upon sucrose selfadministration. Pre-treatment with the KOPr agonist U-50,488 at $10 \mathrm{mg} / \mathrm{kg}$ significantly reduced sucrose pellet self-administration in rats compared to vehicle pre-treatment. U69,593, Ethy-SalA and Me-SalA did not show a significant change in sucrose responding when pre-treated at $0.3 \mathrm{mg} / \mathrm{kg}$. Sucrose responses when pre-treated with vehicle did not differ significantly from mean sucrose responses during baseline sucrose administration sessions. ${ }^{* *}=p<0.001$ (repeated measures one-way ANOVA with Dunnett's post-test: comparison to vehicle). $n=7$ rats for each treatment. 

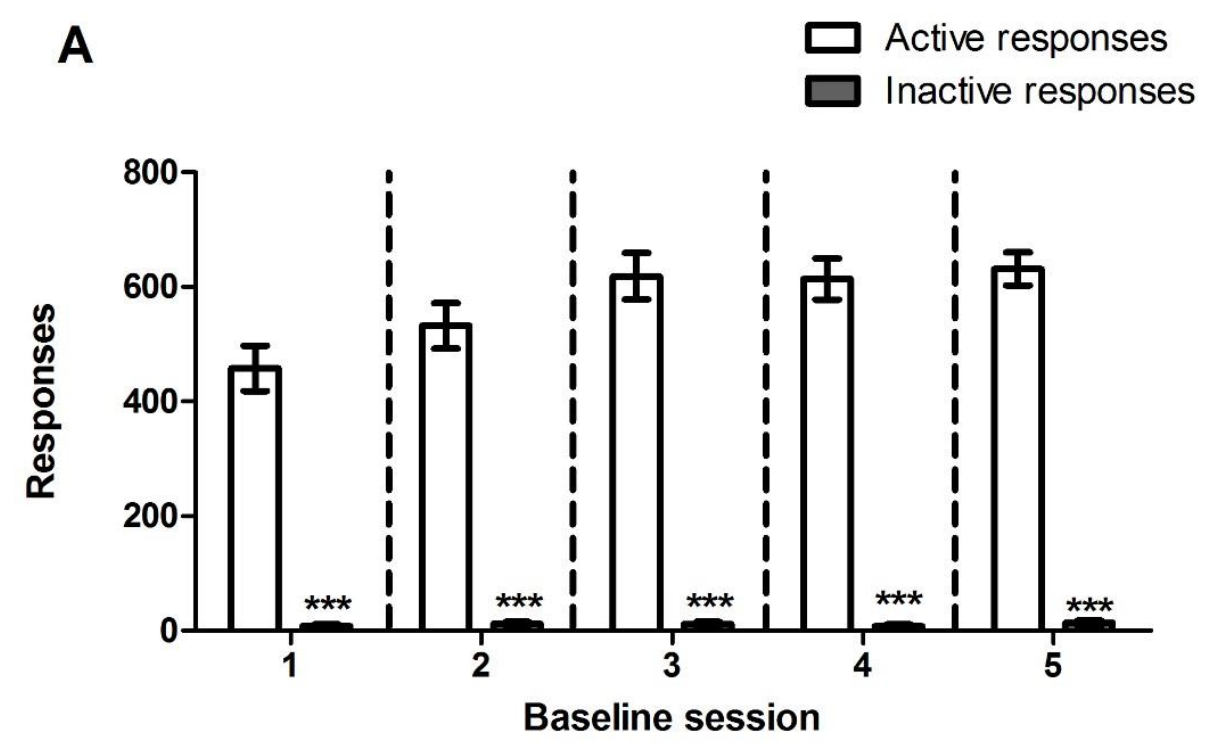

B

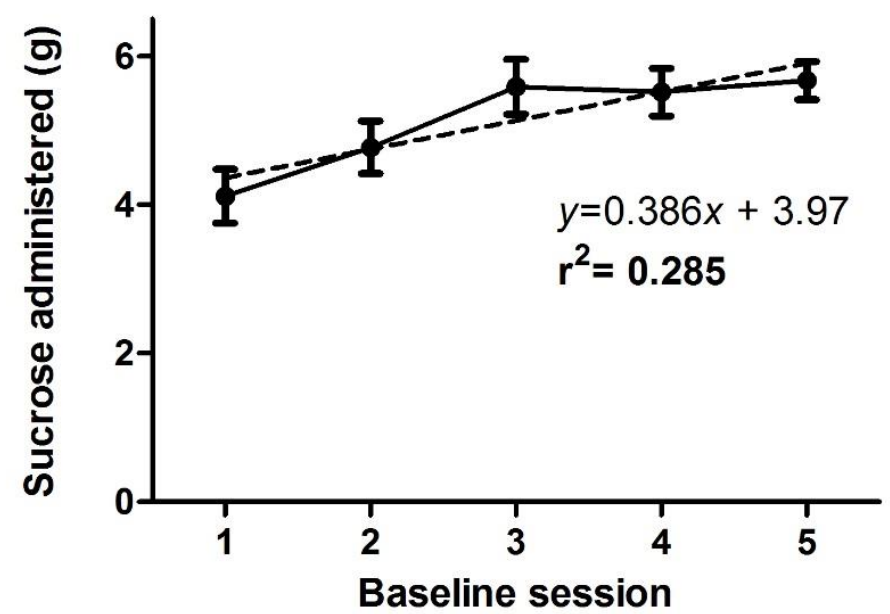

Figure 3.2. Baseline sucrose self-administration responses. The differences in mean active and inactive lever responses for sucrose pellets during 5 three-day baseline sessions $(A)$ were highly significant in all sessions $\left({ }^{* *}=p<0.001\right.$; two-way ANOVA with Bonferroni post-test). Comparison of active baseline lever responses between baseline sessions identified no significant differences ( $p>0.05$; one-way ANOVA). The total amount of sucrose ( $\mathrm{g}$ ) self-administered by rats in each baseline session showed a weak linear increase in dose over time (Pearson correlation coefficient, $r^{2}=0.285$ ). This increase in dose over time was significant ( $p>0.05$; slope not significantly non-zero). $n=7$ rats for each treatment. 


\subsubsection{Locomotor activity profiles of Ethy-SalA and Me-SalA}

Rats treated with $0.3 \mathrm{mg} / \mathrm{kg}$ Ethy-SalA did not show any significant difference in ambulatory counts during the 1 -hour test phase in comparison to vehicle treatment $[t(12)=0.940, p>0.05]$ (Fig. 3.3A). Time course analysis of ambulatory counts (Fig. 3.3B $)$ identified a significant effect of time upon ambulatory counts for both treatment groups $[F(17,204)=23.10, p<0.0001]$, though post-test analysis did not detect any significant differences between treatment groups at any time point. Similar analyses of stereotypic counts also identified no significant difference between vehicle and $0.3 \mathrm{mg} / \mathrm{kg}$ Ethy-SalA treatment $[t(12)=2.094, p>0.05]$, and a significant effect of time upon stereotypic counts $[F(17,204)=20.31, p<0.0001]$ with no significant difference between treatment groups [Bonferroni post-test, $p>0.05]$.

Treatment with Me-SalA $(1.0 \mathrm{mg} / \mathrm{kg})$ also showed no significant change in ambulatory behaviour $[t(12)=0.35, p>0.05]$ or stereotypic behaviour $[t(12)=1.12, p>0.05]$ when compared to vehicle (t-test) (Fig. 3.4A). Time course analysis of ambulatory counts indicated a significant effect of time upon stereotypic counts $[F(17,204)=26.42, p<0.0001]$, with no significant differences between treatment groups [Bonferroni post-test, $p>0.05$ ]. Stereotypic counts for Me-Sal treatment also showed a significant time-related effect $[F(17,204)=17.97, p<0.0001]$ with no significant difference between treatment groups [Bonferroni post-test, $p>0.05$ ] (Fig. 3.4B). 

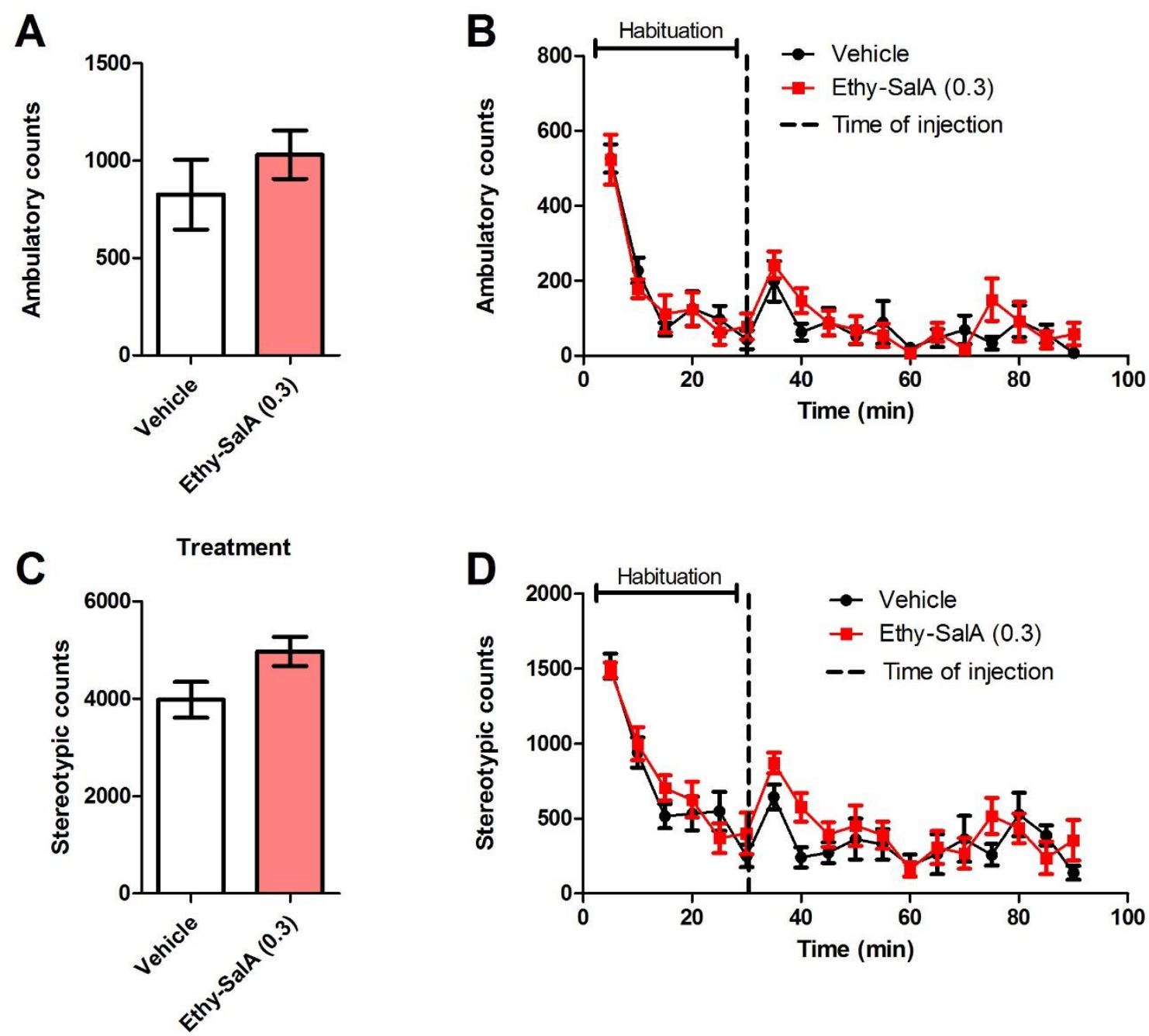

Figure 3.3. Effects of Ethy-SalA on locomotion. Rats treated with $0.3 \mathrm{mg} / \mathrm{kg}$ of EthySalA after $30 \mathrm{~min}$ habituation to an open field activity chamber showed no significant change in total post-habituation ambulatory counts $(A)$ or stereotypic $(B)$ counts compared to vehicle treatment (t-test, $p>0.05$ ). Time-course analysis of ambulatory (C) and stereotypic (D) behaviours with Ethy-SalA treatment showed no significant difference in activity profiles between vehicle and treatment groups during both the habituation and treatment phases of the experiment (two-way ANOVA with Bonferroni post-test, $p>0.05$ ). $\mathrm{n}=7$ rats for each treatment. 

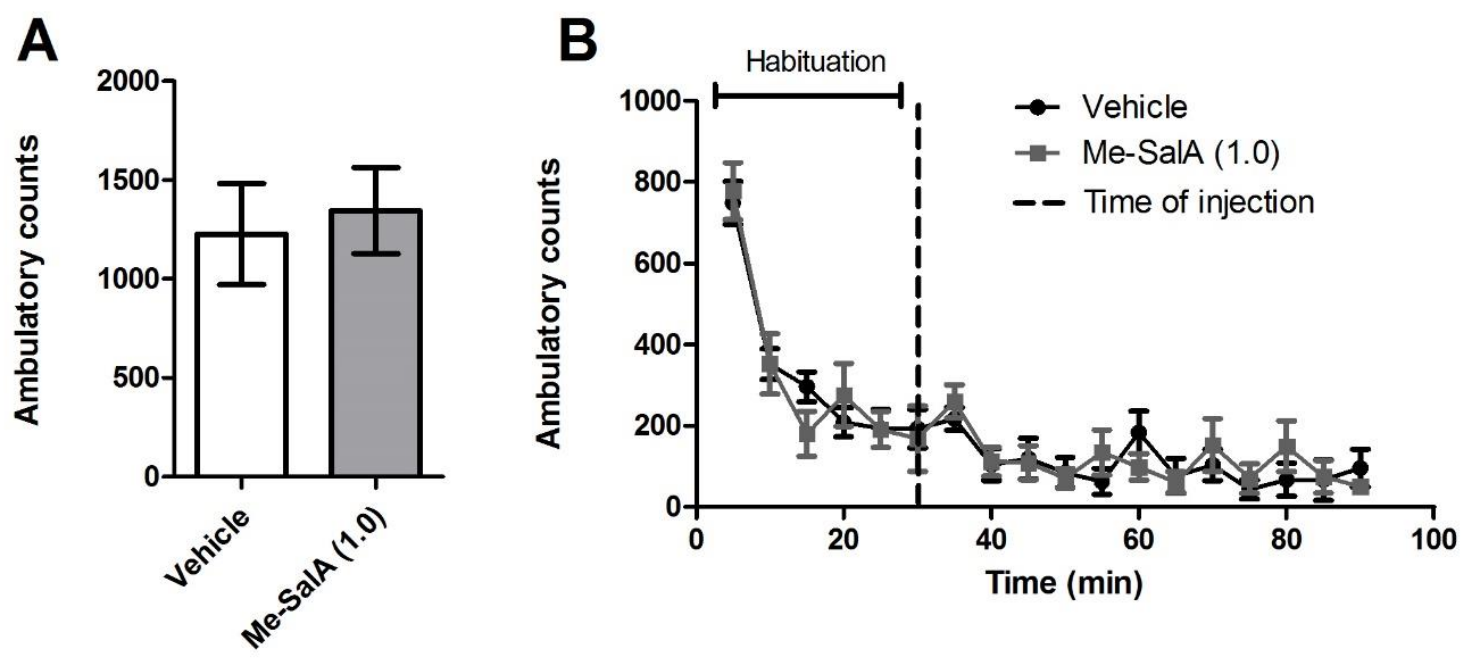

Treatment
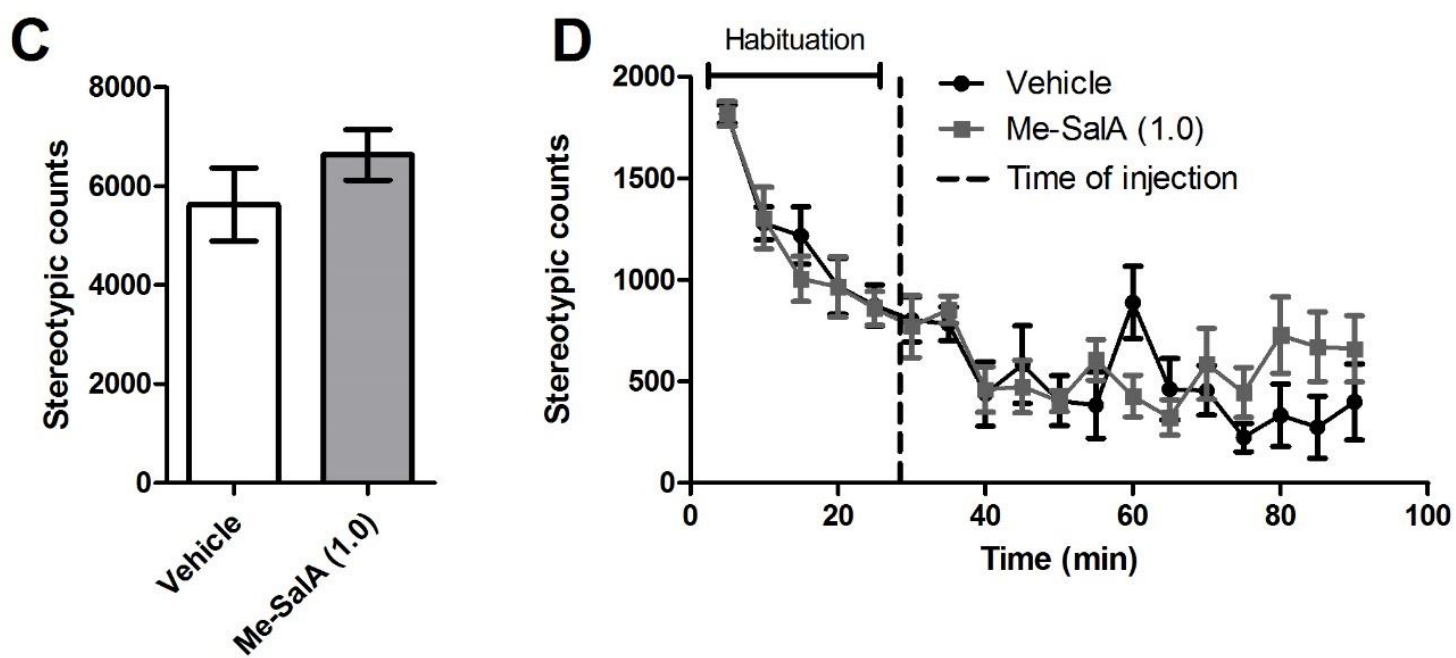

\section{Treatment}

Figure 3.4. Effects of Me-SalA on locomotion. Rats treated with $1 \mathrm{mg} / \mathrm{kg}$ of Me-SalA after 30-min habituation to an open field activity chamber showed no significant change in total post-habituation ambulatory counts $(A)$ or stereotypic $(B)$ counts compared to vehicle treatment (t-test, $p>0.05$ ). Time-course analysis of ambulatory $(C)$ and stereotypic (D) behaviours showed no significant difference in activity profiles between vehicle and treatment groups during both the habituation and treatment phases of the experiment (two-way ANOVA with Bonferroni post-test, $p>0.05$ ). $n=7$ rats for each treatment. 


\subsubsection{Forced Swim Test with acute KOPr agonist treatment}

Comparison of the vehicle-treatment group with Ethy-SalA $(0.3 \mathrm{mg} / \mathrm{kg})$ treatment did not identify any significant differences between the amounts of time rats were scored as displaying immobile $[\mathrm{t}(12)=1.105]$, swimming [t(120=0.563] and climbing $[\mathrm{t}(12)=0.247]$ behaviours (Fig. 3.5A). Rats treated with SalA (0.3 mg/kg) also did not show significant differences in immobile $[\mathrm{t}(12)=1.726]$, swimming $[\mathrm{t}(12)=0.378]$ and climbing $[\mathrm{t}(12)=1.196]$ behaviours compared to vehicle (Fig. 3.5B). 


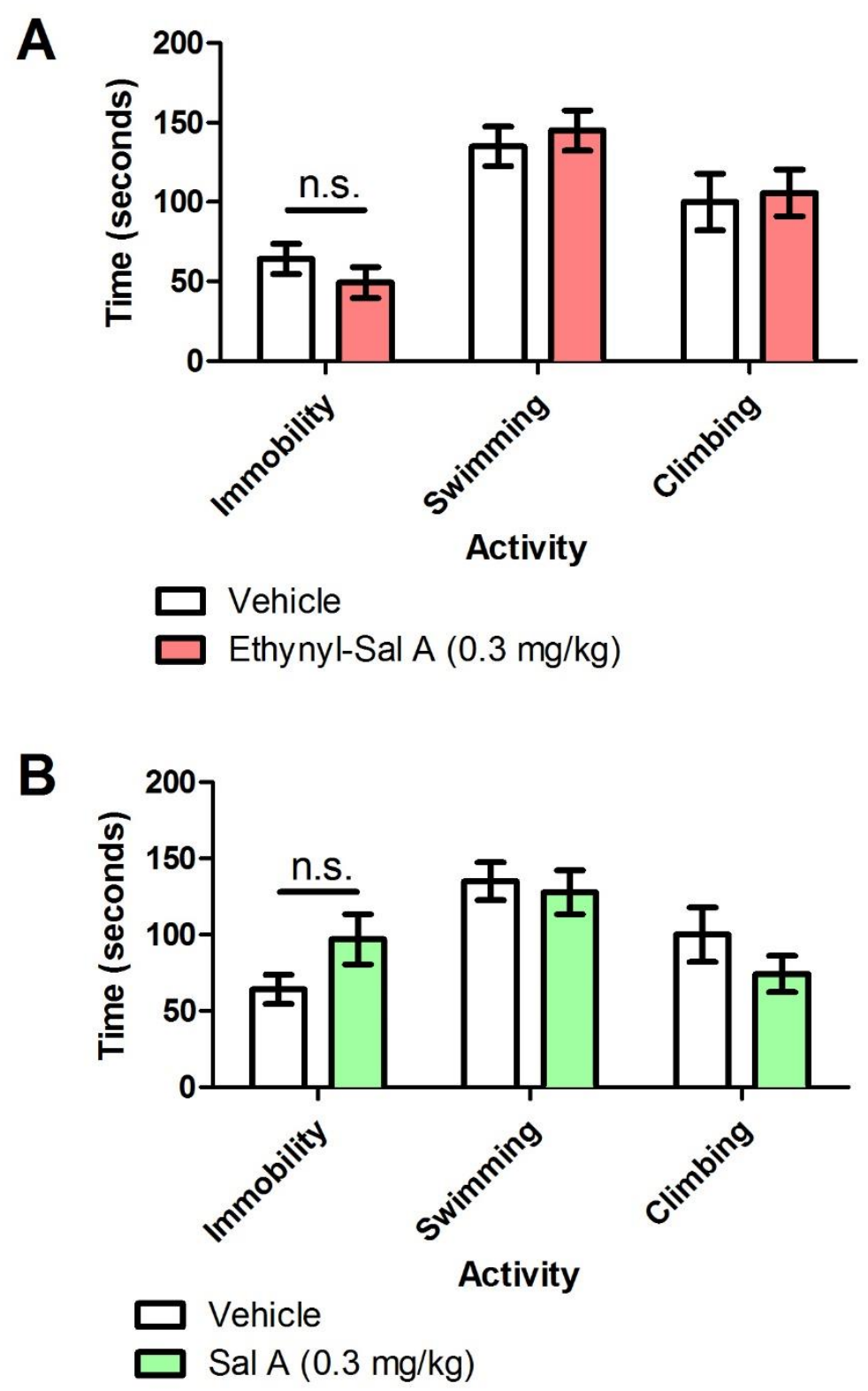

Figure 3.5. Forced Swim Test with acute KOPr agonist treatment. Visual scoring of FST activities showed no significant differences between immobile and active behaviours behaviours between vehicle and Ethy-SalA $(0.3 \mathrm{mg} / \mathrm{kg})(\mathrm{A})$ and SalA $(0.3 \mathrm{mg} / \mathrm{kg})$ (t-test, $p>0.05) . n=9-12$ rats for each treatment. 


\subsection{Discussion}

\subsubsection{Modelling natural reward: sucrose self-administration}

Ethy-SalA has already been shown to attenuate drug-seeking and drug-taking behaviour using two models of cocaine self-administration in rats, indicating that the agonist acts to suppress cocaine reward. To extend the understanding of KOPr activation by Ethy-SalA as a reward inhibitor, sucrose self-administration has been used here as a model of a nondrug or 'natural' rewarding stimulus in rats. Ideally, an anti-addictive pharmacotherapy would inhibit drug-induced reward but not affect natural reward processes, as a complete inhibition of reward sensations could impair addiction therapy, and possibly promote further drug abuse.

Sucrose self-administration has been used in this study is to establish the effects of EthySalA and other KOPr agonists (SalA, U-69,593,) at the effective doses observed to attenuate cocaine-primed reinstatement, or at a comparable dose for an ineffective KOPr agonist (Me-SalA). The agonist U-50,488 was given at a lower dose than the effective cocaine-reinstatement dose (10 mg/kg instead of $30 \mathrm{mg} / \mathrm{kg}$ ) (Morani et al., 2009), as initial tests with this high dose showed complete sedation of rats.

Pre-treatment of SalA, Ethy-SalA, Me-SalA and U-68,593 at $0.3 \mathrm{mg} / \mathrm{kg}$ did not attenuate responses for sucrose pellets in comparison to vehicle, whereas $U-50,488(10 \mathrm{mg} / \mathrm{kg})$ significantly reduced responses for sucrose (Fig. 3.1). This indicates that although SalA and Ethy-SalA interfere with cocaine reward and administration (see Chapter 2), the same doses do not have a detectable effect upon the self-administration of a natural food reward.

Naturally rewarding stimuli are thought to affect the reward pathway in a significantly more complex manner to that of drugs of abuse. A study using rat self-administration models showed that sucrose did not induce long-term potentiation of dopaminergic neurons in the VTA, unlike cocaine (Chen et al., 2008). Other studies have reported that the 
administration of a DA antagonist (pimozide) does not change the palatability of sucrose in rats. Sucrose is still perceived as being sweet and can be discriminated from other stimuli, despite the suppression of rewarding sensorimotor stimulation due to decreased DA signalling (Peciña et al., 1997; Willner et al., 1990). ). It is noticeable that, in this study, rats are shown to self-administer sucrose to a much higher degree than cocaine over a shorter time scale (a 45-minute sucrose administration session as opposed to a 2-hour cocaine administration session) with mean sucrose baseline responses of $570 \pm 31.9$ compared to $190 \pm 15.2$ for cocaine. However, this comparison is not conclusive due to the differences in route of administration (oral of sucrose as opposed to intravenous for cocaine).

\subsubsection{KOPr treatment}

The use of a Latin-square treatment design in this experiment was to mitigate any confounding effects of treatment order or time upon the results of the repeated-measure experiment. Analysis of baseline sucrose intake indicated a significant trend towards an increase in sucrose dose over time (Fig. 3.2B). As the rats used in this experiment were maintained at $85 \%$ of their initial pre-training weight throughout sucrose administration, the effect observed is likely to be age-related rather than weight related, or as a result of multiple KOPr agonist injections. This effect very likely distorts some of the results obtained, and makes a repeated measures analysis less viable. However, the counterbalanced treatment design in this experiment should at least partially account for this effect.

The observed increase in sucrose dosage contrasts with the finding that cocaine self-

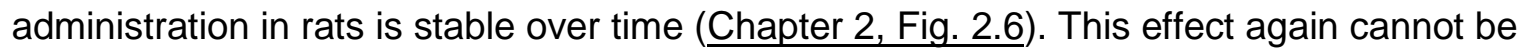
directly compared due to differences in the routes of administration, but may further 
support the existence of differences in drug-reward and natural-reward processing within the brain.

\subsubsection{Sedative side-effects: locomotor activity testing}

Open field locomotion tests were used to identify the potential sedative effects of EthySalA and Me-SalA, both as part of general side-effect profiling for the agonists and in order to distinguish attenuation of response due to a reduced reward cue in cocaine-primed selfadministration testing from attenuation of response due to test subject sedation. Sedative effects have been shown for SalA in rats $(2.0 \mathrm{mg} / \mathrm{kg}$, i.p.) (Chartoff et al., 2008) and in rhesus monkeys $(0.1 \mathrm{mg} / \mathrm{kg}$, i.v.) (Butelman et al., 2009), but at doses far greater than those used in self-administration experiments. Low doses of SalA analogues (0.1-1.0 $\mathrm{mg} / \mathrm{kg}$, i.p.) have not shown significant sedative effects (Simonson et al., 2015; Bosch, PhD thesis 2014; Ewald, PhD thesis 2015), suggesting that sedative effects are only significant with high doses of a KOPr agonist. Reduced locomotor activity was not observed with Ethy-SalA (0.3 mg/kg, i.p.) or Me-SalA (1.0 mg/kg, i.p.) treatment at the maximum doses used for cocaine-primed reinstatement testing, indicating that treatment with these compounds should not affect the ability of rats to lever-press during selfadministration.

\subsubsection{Pro-depressive side-effects: the Forced Swim Test}

The Forced-Swim Test (FST) was originally designed as an assay to test the antidepressant effects of novel compounds (Porsolt et al., 1977), but has in some studies been re-purposed to examine pro-depressive effects (Carlezon et al., 2006; Morani et al., 2012). The amount of time that a test animal spends immobile is used as an approximation of a 
depressive or despairing state, and changes to immobility time in comparison to vehicle treatment have been used to gauge the anti-depressive or pro-depressive effects of compounds administered to rats or mice. 24 hours before the test day, rats are exposed to the water-filled FST chamber for 15 minutes. This habituation step is to reinforce the stress-inducing effects of continuous swimming, and to 'teach' the rat that escape from the chamber during the test is not possible, enhancing the likelihood of observing behavioural despair during the 5-minute test phase.

In this experiment, a $0.3 \mathrm{mg} / \mathrm{kg}$ treatment of Ethy-SalA did not significantly increase or decrease immobility, swimming/medium activity or climbing/high activity times in comparison to vehicle, indicating that Ethy-SalA does not induce pro-depressive effects at a low dose.

SalA has previously been shown to display pro-depressive effects in rats with single acute (Morani et al., 2012) and acute multiple treatments (Carlezon et al., 2006), and so was used as a positive control in this experiment at a dose of $0.3 \mathrm{mg} / \mathrm{kg}$. However, SalA did not cause a significant increase in immobility, conflicting with previous acute administration data (Morani et al., 2012), which calls into question the reliability of these results. Climbing/high activity and swimming/medium activity times were unusually high in comparison to literature FST results. One possible explanation for these observations is that all of the rats used for FST had previous experimental histories (in CPP, novel-object recognition tests, and elevated-plus maze tests) and many had received either KOPr agonist or vehicle treatment, whereas the rats used by both Carlezon et al. (2006) and Morani et al. (2012) were naïve to KOPr agonists and were assumed to have no previous experimental history. Carlezon et al. (2006) also gave rats three injections of SalA in the 24 hours prior to testing, whereas the FST protocol used here and by Morani et al. (2012) uses only a single pre-treatment. 
The re-use of rats in this thesis was designed to minimise unnecessary wastage, according to the recommendations outlined by the National Centre for the Replacement, Refinement and Reduction in Animal Research (UK). A period of at least 1 week was allowed between the end of the previous experiment and the start of FST, to allow any residual KOPr agonist to pass out of the animal. Recent studies have indicated that a single acute injection of SalA may have much longer-lasting neurophysiological effects in rats than previously thought (Chartoff et al., 2015), so that even a single injection of a short-acting KOPr agonist may have significant long-term effects upon the rodent brain. It is therefore possible that previous exposure to KOPr agonists and/or previous experimental experience may be protective against the pro-depressive effects of the FST.

\subsubsection{Limitations and future directions}

This study has examined only the potential reward-attenuating properties of KOPr agonist treatment, but has not taken into account the more complex interaction between the KOPr system and natural reward. KOPr activation has been linked to increased appetite and administration of natural rewards. Rats treated with low doses of the KOPr agonist U50,488 (215 nM infusion) have shown increased consumption of high-fat foods (Ookuma et al., 1997), an effect which is opposed by administration of the KOPr antagonist norBNI (Arjune \& Bodnar, 1990). The link between KOPs and appetite appears to be the proreward and appetite-stimulating peptide hormone orexin, which has recently been identified to have a potentially important role in the positive regulation of cocaine reward (Hollander et al., 2012). Despite their apparent opposing functions, both dynorphin and orexin have been observed to be released in response to electrical hypothalamic stimulation (Li \& van den Pol, 2006), and recent research has identified that these two peptides are in fact packaged within the same synaptic vesicles in the hypothalamus (Muschamp et al., 2014). The increase in sucrose administration over time observed in 
this study is possibly related to the appetite-stimulating properties of KOPr agonists. The interplay of these two signalling pathways complicates the traditional anti-reward properties of the KOP system, and merits further investigation.

High doses of both SalA and Ethy-SalA at $2 \mathrm{mg} / \mathrm{kg}$ (as used in cocaine self-administration testing; see Section 2.1.6) were not tested in the sucrose-administration paradigm, limiting conclusions that SalA and Ethy-SalA display cocaine-reward specific effects. At higher doses, it is possible that SalA and Ethy-SalA could act as general inhibitors of all reward processes, and future tests should include doses higher than those tested here $(0.3$ $\mathrm{mg} / \mathrm{kg})$.

The locomotor activity assay used here is limited by the lack of a positive sedative control, which would confirm whether the test has the ability to accurately detect sedation in rats. By measuring only total activity, the locomotor activity test used here does not take into account more specific sedative effects, such as balance and co-ordination. One such assay that does explore these effects is the rotarod performance test (Dunham \& Miya, 1957), where rats or mice are trained to negotiate a constantly rotating wheel, and then tested for the ability of potentially sedative drugs or disease states to alter their performance on the wheel. Rotarod testing of rats treated with Ethy-SalA and Me-SalA would complement the existing data and confirm if any either of the compounds have significant motor and coordination side-effects.

As well as test for sedation, locomotor activity in rats can be used to gauge the hallucinogenic properties of novel drugs. SalA is a known potent hallucinogen, and it could be expected that the derivatives Ethy-SalA and Me-SalA would also display hallucinogenic-like effects. Classic hallucinogens such as LSD and mescaline have been observed to significantly alter exploratory behaviours and stereotypic locomotor behaviours, with characteristic suppression of exploratory behaviour (Adams \& Geyer, 1985), reduced interaction with novel stimuli (Geyer et al., 1979), and rotational movement 
(Fleisher \& Glick, 1979) observed in Wistar and Sprague-Dawley rats. However, these tests have been established using serotonergic hallucinogens, from which SalA shows significant behavioural differences in rats (Killinger et al., 2010). Zebra-fish have been recently identified as an alternative model for hallucinogenic effects, with characteristic changes in immobility-versus-activity and swimming and diving patterns identified for the classical hallucinogens LSD (Grossman et al., 2010), mescaline, and phenylcyclidine (Kyzar et al., 2012); and also for the novel hallucinogens ibogane (a mixed MOR, KOPr and 5-HT receptor agonist) (Cachat et al., 2013) and SalA (Braida et al., 2007). Tests using a zebra-fish model could confirm whether Ethy-SalA and Me-SalA display significant hallucinogenic effects.

The results of FST testing show here are not conclusive, due to the lack of an effective positive control. Repetition of this experiment with larger numbers and with experimentally naïve animals could improve upon this finding.

The FST shows some limitations as a model of behavioural despair in rats. In the original proposed test, immobile behaviour was taken as a measure of a despair-like state, due to test subjects learning during the pre-test that escape was not possible (Porsolt et al., 1977). A study comparing groups of rats allowed to escape the FST chamber during the pre-test and rats with no chance of escape showed no differences in immobility times during the test phase (O'Neill \& Valentino, 1982), suggesting that the experience of the pre-test may not significantly influence test behaviour. Some uncertainty also exists as to whether the immobility observed during the test phase is truly due to a despair-like state, or whether the response is more an adaptation to a familiar environment (Borsini \& Meli, 1988; Hawkins et al., 1978 ). The tail-suspension test (TST) is an alternative prodepressive model performed in mice (Steru et al., 1985). After treatment, subjects are suspended by the tail and isolated from any means of support, with immobile behaviour during testing also being used as an approximation of despair. The test does not require a pre-test phase, avoiding the confounds associated with prior exposure to the test 
environment, is generally short (around 6 minutes), and also avoids the potentially confounding effects of hypothermia due to immersion in water (Cryan et al., 2005). The TST could therefore be used to corroborate FST results, as a second measure of potential anti-depressant or pro-depressive effects.

\subsubsection{Conclusions}

Acute treatments of the novel KOPr-agonists Ethy-SalA and Me-SalA were shown to have no effect on sucrose self-administration (at $0.3 \mathrm{mg} / \mathrm{kg}$ ), or affect basal locomotor activity in Sprague-Dawley rats at the highest doses used for cocaine-primed reinstatement testing (0.3 and $1.0 \mathrm{mg} / \mathrm{kg}$ respectively). Ethy-SalA $(0.3 \mathrm{mg} / \mathrm{kg})$ also shows no significant prodepressive or anti-depressive effects in FST with comparison to vehicle treatment. 


\section{Chapter 4 : Cellular effects of Ethy-SalA: modelling dopamine re-uptake}

KOPr agonists, including SalA and many of its chemical derivatives, have been shown in cellular and ex vivo tissue models to influence re-uptake of DA via activation of the KOPr (Kivell et al., 2014; Simonson et al., 2015). The dye trans-4-[4-(dimethylamino)styryl]-1methylpyridinium (ASP+) acts as a substrate for monoamine transporters, including DAT, and fluoresces strongly in a lipid environment (Magrassi et al., 1987; Schwartz et al., 2003). Transfected cells expressing DAT show linear uptake of ASP+, which accumulates within the cytoplasm and can be detected using confocal microscopy (Zapata et al., 2007). Measures of ASP+ uptake have been used to identify changes to DAT function in cells coexpressing KOPr and DAT. This chapter examines the cellular effects of Ethy-SalA upon DAT function using an jn vitro cellular model of DA re-uptake, in order to determine a mechanism of action for the agonist's anti-cocaine effects.

\subsection{Methods}

\subsubsection{Cell culture materials and solutions}

Sterile plasticware, including cryovials, Falcon tubes (15 and $50 \mathrm{~mL})$, T25 $\left(25 \mathrm{~cm}^{2}\right)$ and T75 $\left(75 \mathrm{~cm}^{2}\right)$ flasks were obtained from Becton Dickinson Ltd. (Auckland, New Zealand). Confocal glass-bottomed Fluorodishes were obtained from Coherent Scientific (WPI Inc., 
Victoria, Australia). Dubecco's Modified Eagle's Medium (DMEM; Invitrogen), penstrep antibiotic (penicillin G sodium 5000 units $/ \mathrm{mL}$ and streptomycin sulphate 5000 units $/ \mathrm{mL}$ in 0.85\% saline), Lipofectamine 2000 (Invitrogen), Opti-MEM 1 medium (Invitrogen), bovine serum albumin and trypsin was obtained from Life Technologies Ltd. (Auckland, New Zealand). Fetal calf serum (FCS) was obtained from ICP Biologicals, (Auckland, New Zealand). Phosphate-buffered saline (PBS; $137 \mathrm{nM} \mathrm{NaCl}, 2.7 \mathrm{mM} \mathrm{KCl}, 8.1 \mathrm{mM} \mathrm{Na} \mathrm{HPO}_{4}$, $\left.1.5 \mathrm{mM} \mathrm{KH}_{2} \mathrm{PO}_{4}, \mathrm{pH} 7.4\right)$ and Krebs buffer (130 mM NaCl, $1.3 \mathrm{mM} \mathrm{KCl}, 2.2 \mathrm{mM} \mathrm{CaCl} 2,1.2$ mM MgSO${ }_{4} .6 \mathrm{H}_{2} \mathrm{O}, 1.2 \mathrm{mM} \mathrm{KH}_{2} \mathrm{PO}_{4}, 10 \mathrm{mM}$ HEPES, $10 \mathrm{mM}$ D-glucose, $\mathrm{pH}$ 7.4) were prepared using lab reagents and sterilised (autoclaved and filtered) before use. Trans-4[4-(dimethylamino)styryl]-1-methylpyridinium (ASP+) iodide and norbinaltorphimine (norBNI) was obtained from Tocris Bioscience (Bristol, UK). Anti-myc monoclonal mouse antibodies and goat anti-mouse Cy5 antibodies were obtained from Abcam (Melbourne, Australia). The YFP-hDAT and myc-rKOPr plasmids used in this study were kindly donated by L. Devi (Jordan \& Devi, 1999), and J. Javitch (Daws et al., 2002) respectively.

\subsubsection{Cell culture- Human Embryonic Kidney cells (HEK-293)}

HEK-293 cells were grown and passaged using sterile plasticware in a sterile biological safety cabinet (AES Environment PTY, Auburn, Australia). Cells were cultured in a standard DMEM solution containing 10\% (v/v) Fetal Calf Serum and 1\% (v/v) penstrep antibiotic. Cell cultures were stored and grown in a Heracell incubator (Kendro Laboratory Products, $\mathrm{GmbH}$, Germany) at $37^{\circ} \mathrm{C}$ in humid conditions and with $5 \%$ carbon dioxide.

An adherent immortalised Human Embryonic Kidney cell line (HEK-293) was used for all cellular experimentation. HEK-293 cell stocks were stored in cryovials in liquid nitrogen, and thawed for use. Vials were defrosted in a $37^{\circ} \mathrm{C}$ water bath containing metal beads, and cells were added to a $15 \mathrm{~mL}$ Falcon tube containing $10 \mathrm{~mL}$ of standard DMEM and centrifuged at $1250 \mathrm{rpm}, 300 \mathrm{~g}$ for $5 \mathrm{~min}$. Pelleted cells were re-suspended in $7 \mathrm{~mL}$ of 
standard DMEM and placed in a sterile T25 flask and allowed to grow for approximately 2 days until cells were $80-90 \%$ confluent, at which point the cells were passaged.

Cells were passaged by removing the culture media and washing with $5 \mathrm{~mL}$ of $1 \mathrm{x}$ phosphate buffered saline (PBS). Cells were then incubated with $1 \mathrm{~mL}$ trypsin for 3-5 minutes, until cells had visibly detached from the bottom of the flask. $5 \mathrm{~mL}$ of standard DMEM was added to the flask to inactivate the trypsin, and the mixture pipetted multiple times over the surface of the flask to wash any remaining cells from the flask. The cell suspension was the centrifuged at $1250 \mathrm{rpm}, 300 \mathrm{~g}$ for $5 \mathrm{~min}$, and the pelleted cells resuspended in $2 \mathrm{~mL}$ of standard DMEM. Cells were re-seeded at $1 \mathrm{~mL}$ in a total of $15 \mathrm{~mL}$ standard DMEM in a T75 tissue culture flask. For further passaging of T75 flasks, cells were treated with $2 \mathrm{~mL}$ of trypsin, and the pelleted cells re-suspended in $5 \mathrm{~mL}$ of standard DMEM.

\subsubsection{HEK-293 co-transfection}

In preparation for transfection, cells were passaged and plated on glass-bottomed $35 \mathrm{~mm}$ diameter Fluorodishes at a concentration of $3.0 \times 10^{5} \mathrm{cells} / \mathrm{mL}$ in $1 \mathrm{~mL}$ of standard DMEM. To obtain a specific concentration for plating, the number of live cells in suspension was counted using $0.4 \%$ trypan blue (Sigma-Aldrich, Auckland, NZ) and a standard haemocytometer. 24 hours after plating the culture medium was removed and replaced with $1 \mathrm{~mL}$ of penstrep-free DMEM (10\% FCS only). To give transfection solution for a single plate, $3 \mu \mathrm{L}$ of Lipofectamine 2000 was combined with $47 \mu \mathrm{L}$ of Opti-MEM 1 medium (Invitrogen) and incubated for $5 \mathrm{~min}$ at room temperature. In a separate tube, $1.8 \mu \mathrm{g} / \mathrm{mL}$ of myc-tagged rat KOPr (myc-rKOPr) plasmid DNA, and $0.8 \mu \mathrm{g} / \mathrm{mL}$ of yellow fluorescent protein-tagged human DA transporter (YFP-hDAT) plasmid DNA were made up in a total volume of $50 \mu \mathrm{L}$ of Opti-MEM 1. After the 5 min incubation, both tubes were combined and 
incubated at room temperature for $20 \mathrm{~min}$. Dishes were then treated with $100 \mu \mathrm{L}$ of the transfection solution, and incubated for 48 hours.

\subsubsection{Confocal microscopy: trans-4-[4-(dimethylamino)styryl]-1- methylpyridinium (ASP+) uptake}

Transiently co-transfected HEK293 cells were visualised with an Olympus Fluoview FV1000 confocal laser scanning biological microscope (inverted model 1X81), using FV10-ASW 4.0 software (Olympus Pty. Ltd., Melbourne, Australia). A 10 mM stock solution $\mathrm{ASP}+$ was made using sterile Krebs buffer. This stock was further diluted to a concentration of $10 \mu \mathrm{M}$ in Krebs Buffer, and incubated in a $37^{\circ} \mathrm{C}$ water bath.

Media was aspirated from plated cells, and replaced with sterile KREBs buffer at $37^{\circ} \mathrm{C}$. A glass-bottomed $35 \mathrm{~mm}$ diameter dish containing YFP-hDAT and myc-rKOPr transfected cells was placed in an insulated heating chamber (INU-21LCS-f1 model; Tokau Hit Co. Ltd, Japan) at $37^{\circ} \mathrm{C}$, filled with distilled water perfused with $5 \% \mathrm{CO}_{2}$. Cells were observed at $60 \mathrm{X}$ magnification using a silicon immersion objective. YFP-hDAT fluorescence was observed using a $473 \mathrm{~nm}$ laser (YFP filter: excitation at $473 \mathrm{~nm}$, detection at $527 \mathrm{~nm}$ ) at $0.7 \%$ laser power and high voltage settings $(\mathrm{HV})$ of $700 \mathrm{~V}$. ASP+ fluorescence was observed using a $558 \mathrm{~nm}$ laser (ASP+ filter: excitation at $570 \mathrm{~nm}$, detection at $670 \mathrm{~nm}$ ) at $0.5 \%$ laser power and HV of $560 \mathrm{~V}$. A differential interference contrast (DIC) filter was used to visualise all cells in the field of view. The microscope pinhole was opened to $500 \mu \mathrm{m}$ to reduce confocality, allowing near-total cellular accumulation of ASP+ to be visualised. The viewing software was programmed take 120 images at five-second intervals in a single field of view, for a total time-course of 10 minutes. 
Once a field of view was chosen, with at least $50 \%$ of the cells fluorescing and visible in the YFP confocal channel, Krebs solution was aspirated from the cells, and the timecourse started. Once the first image had been taken, $1 \mathrm{~mL}$ of $10 \mu \mathrm{M}$ ASP + was carefully added to the dish. Baseline ASP+ uptake was recorded up to 300 seconds, after which 1 $\mu \mathrm{L}$ of KOP agonist or vehicle (100\% DMSO) was added to the dish in the vicinity of the field of view and combined with the Krebs/ASP+ medium by gently pipetting up and down. Treated cells were then imaged for a further 300 seconds.

KOP agonists were prepared at a concentration of $10 \mathrm{mM}$ and $1 \mathrm{mM}$ in pure DMSO, so that when $1 \mu \mathrm{L}$ of agonist solution was added to the $1 \mathrm{~mL}$ of KREBS solution the final concentration of agonist was approximately $10 \mu \mathrm{M}$ and $1 \mu \mathrm{M}$ respectively. For experiments requiring norBNI pre-treatment, cells were incubated in $1 \mathrm{~mL}$ of Krebs solutions containing $1 \mu \mathrm{M}$ of norBNI (Tocris Bioscience, UK) prior to the start of confocal experimentation.

$\mathrm{ASP}+$ uptake was analysed by manual selection of regions-of-interest (ROIs) encompassing YFP-hDAT-expressing cells, and plotting Arbitrary Fluorescent Units (AFUs) over time, using the Series Analysis function of the FV10-ASW 4.0 software (Olympus Pty. Ltd, Australia). ROls were excluded from analysis if the regions initially showed YFP-hDAT fluorescence of less than 100 AFUs, and if the plot of ASP+ fluorescence showed significant deviation from a linear uptake, as this indicated that the selected region contained a cell which had shifted or moved out of focus. The rate of ASP+ uptake for ROls was approximated by plotting AFU values for a 60 -second period of time prior to addition of KOPr agonist (typically from 200-260 seconds), and then plotting the fluorescence of multiple overlapping 60 -second periods (20-second overlap, with a minimum of 5 periods plotted) after agonist addition. A trendline was fitted to each plot using Linear regression software (Prism 5.0, GraphPad, La Jolla, USA). The maximal gradient observed post-agonist addition was compared to the pre-agonist gradient using the following formula:

$\%$ change in uptake $=\left[\frac{(\text { gradient } \text { post }- \text { agonist })}{(\text { gradient } \text { pre }- \text { agonist })} \times 100\right]-100$ 
All treatments (excepting norBNI treatment) were performed over a minimum of 3 separate transfections, with 2-3 plates per transfection for each treatment condition.

\subsubsection{Confocal visualisation of myc-rKOPr expression}

KOPr immunostaining was performed 72 hours after plating and 48 hours after cotransfection. Cells were fixed with ice-cold methanol/acetone (1:1) for $7 \mathrm{~min}$, and then washed 3 times, at 5 min per wash, in Phosphate-Buffered Saline (PBS). Cells were then blocked with $0.25 \%$ Tween 20, 1\% Bovine Serum Albumen (BSA) in PBS for 30 min at room temperature. The blocking solution was aspirated, and anti-myc monoclonal mouse antibody in 1\% BSA in PBS at a dilution of $1: 20$ was incubated with the cells at $4^{\circ} \mathrm{C}$ for 12 hours. After incubation, the antibody solution was aspirated, and the cells washed 3 times with $0.25 \%$ Tween 20 in BSA, at 5 min per wash. Goat anti-mouse Cy5 antibody at a dilution of 1:100 was added to the cells and incubated at room temperature for one hour. The antibody solution was replaced with PBS and stored at $4^{\circ} \mathrm{C}$ in the dark prior to confocal imaging. YFP-hDAT and Cy5-immunolabelled myc-rKOPr-expressing cells were visualised by confocal microscopy (see above) using a $493 \mathrm{~nm}$ and $693 \mathrm{~nm}$ lasers with YFP (see Section 4.1.3) and Cy5 (excitation at $635 \mathrm{~nm}$, detection at $664 \mathrm{~nm}$ ) filters, at 40x magnification (silicon objective) (Olympus Fluoview FV1000 microscope).

\subsubsection{Statistical analysis}


All statistical analysis was performed using GraphPad Prism 5.0 software. Mean ASP+ uptake increases post-KOPr agonist addition were compared using a one-way ANOVA with Dunnett's multiple comparison post-test (comparison to vehicle). Results were considered to show significance if the $p$-value for statistical tests was $<0.05$. Numerical results are reported in-text as Mean \pm Standard Error of Mean.

\subsection{Results}

\subsubsection{Co-expression of myc-rKOPr and YFP-hDAT in transfected HEK-293 cells}

Visualisation of YFP-hDAT and myc-rKOPr, using auto- and immunofluorescence respectively, indicates that co-transfection with YFP-hDAT and myc-rKOPr plasmids induces co-expression of YFP-DAT and myc-KOPr protein in HEK-293 cells. Overlays of YFP-hDAT and Cy5-immunostained myc-rKOPr images show expression of both proteins occurring within the same cells (Fig. 4.1). 


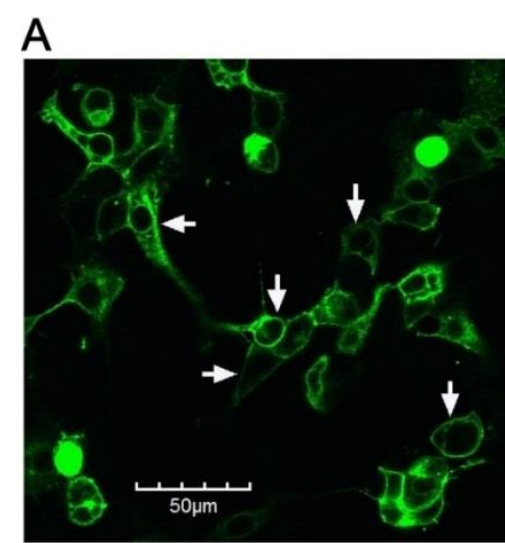

DAT

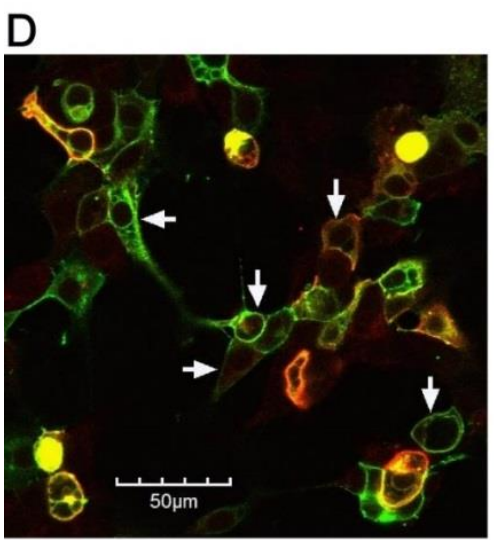

Overlay 1 (DAT/KOPr)

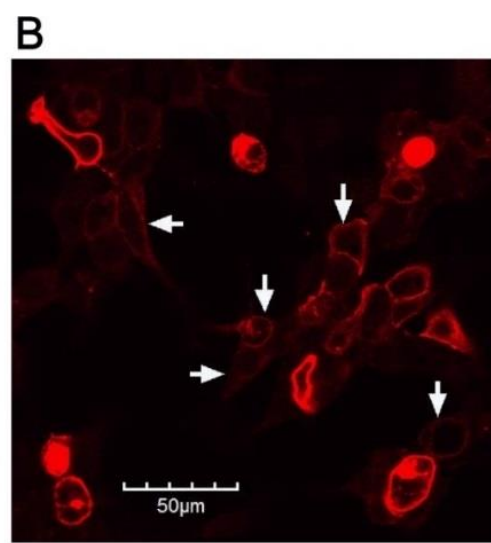

$\mathrm{KOPr}$

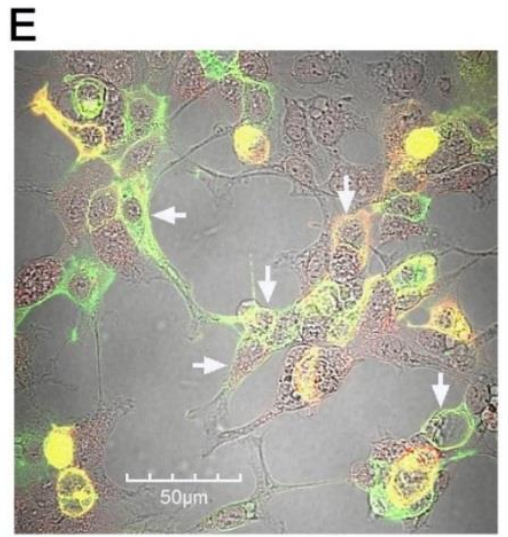

Overlay 2 (DAT/KOPr/DIC)

$\mathrm{E}$

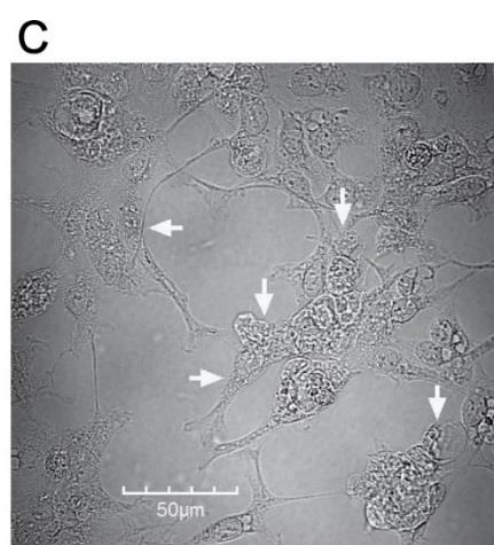

DIC

Figure 4.1. Co-expression of myc-rKOPr and YFP-hDAT in transfected HEK293 cells. Confocal microscopy images of auto-fluorescent YFP-hDAT (A) and Cy5stained myc-rKOPr (B) expression. White arrows indicate regions of YFP-hDAT and mycrKOPr fluorescence. The marked areas of fluorescence in $A$ and $B$ correspond to cellular structures in DIC images ( $C$ and E), with overlap between regions of YFP-hDAT and mycrKOPr expression (D). 


\subsubsection{Characterisation of ASP+ uptake in DAT/KOPr co-transfected HEK 293}

cells

Cells expressing YFP-hDAT show marked accumulation of fluorescent ASP+ over time, whereas non-expressing cells do not (Fig. 4.2) . ASP+ uptake in representative YFP-hDATexpressing show strong linear uptake approximately $100 \mathrm{~s}$ after addition of $\mathrm{ASP}+[\mathrm{Pearson}$ correlation coefficient, $\mathrm{r}^{2}>0.98$ ] up until $300 \mathrm{~s}$. Linear uptake continued after addition of 1 $\mu \mathrm{L}$ of vehicle (100\% DMSO) or KOPr agonist to the cells at $300 \mathrm{~s}$, though changes in the slope of ASP+ uptake can be observed (Fig. 4.3). 
A

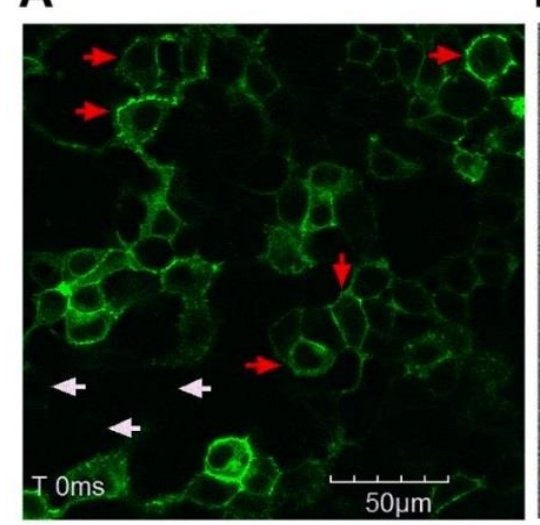

DAT

D

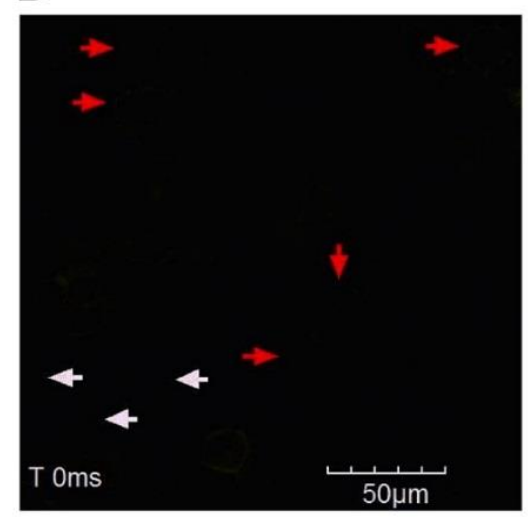

G

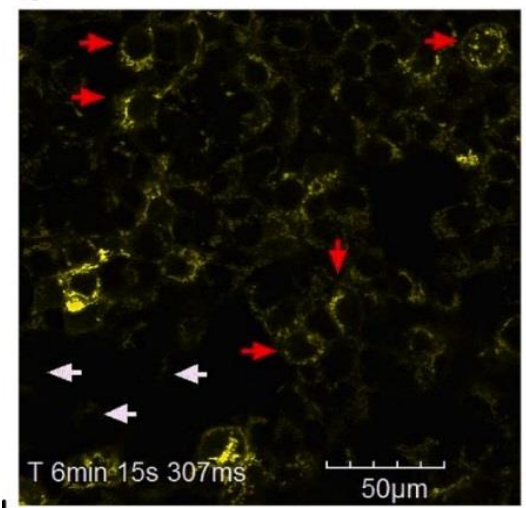

B

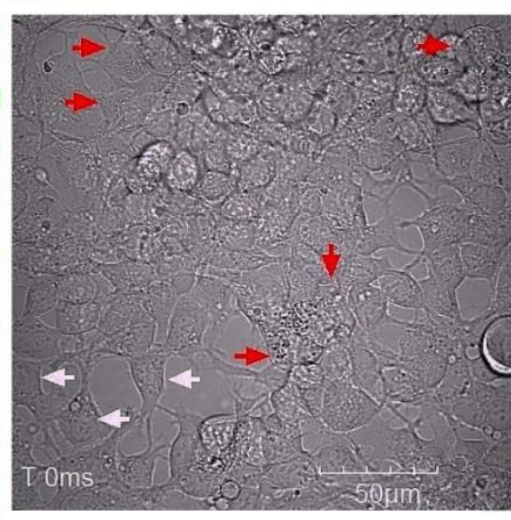

DIC

E

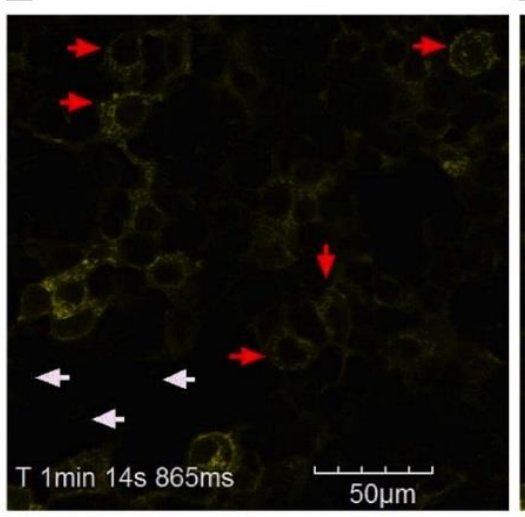

H

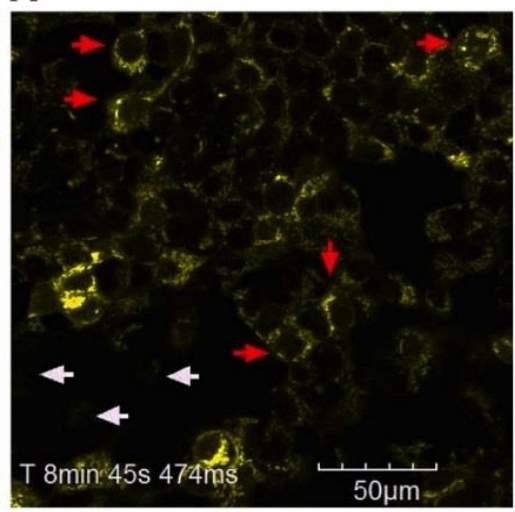

C

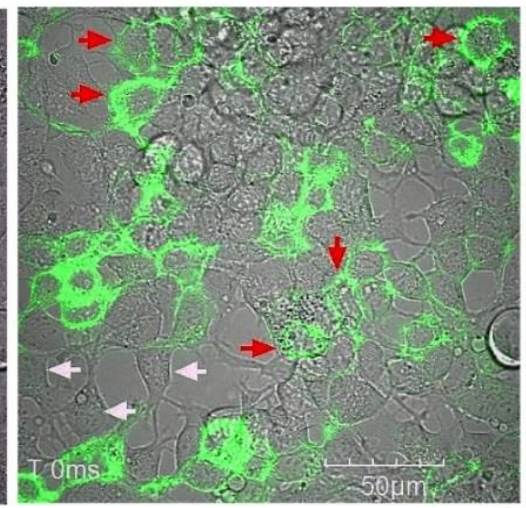

Overlay (DAT/DIC)

F

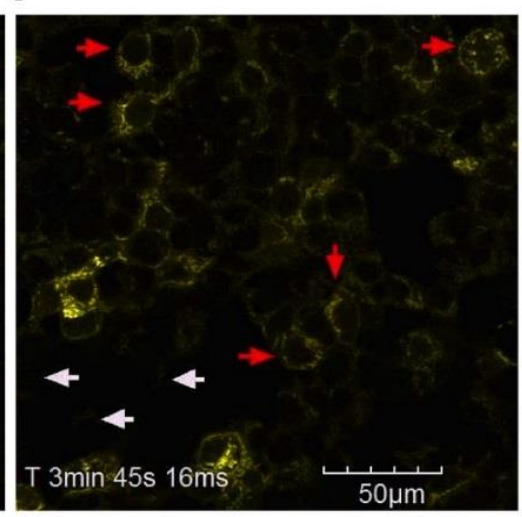

ASP+

Figure 4.2. Time course images of ASP+ uptake in co-transfected DAT/KOPr HEK293 cells. Images A-C represent YFP-hDAT expression at time $=0$ seconds, prior to ASP+ addition. YFP-hDAT-expressing (red arrows) and non-expressing cells (white arrows) are indicated. Images D-H represent accumulation of ASP+ within the same sample of cells from $0 \mathrm{~s}$ to 8 minutes 45 seconds. Fluorescence increases within cells expressing YFPhDAT (red arrows), while non-expressing cells do not show visible accumulation (white arrows). The punctate fluorescence observed in labelled cells is a result of mitochondrial binding of $\mathrm{ASP}+$. 
A

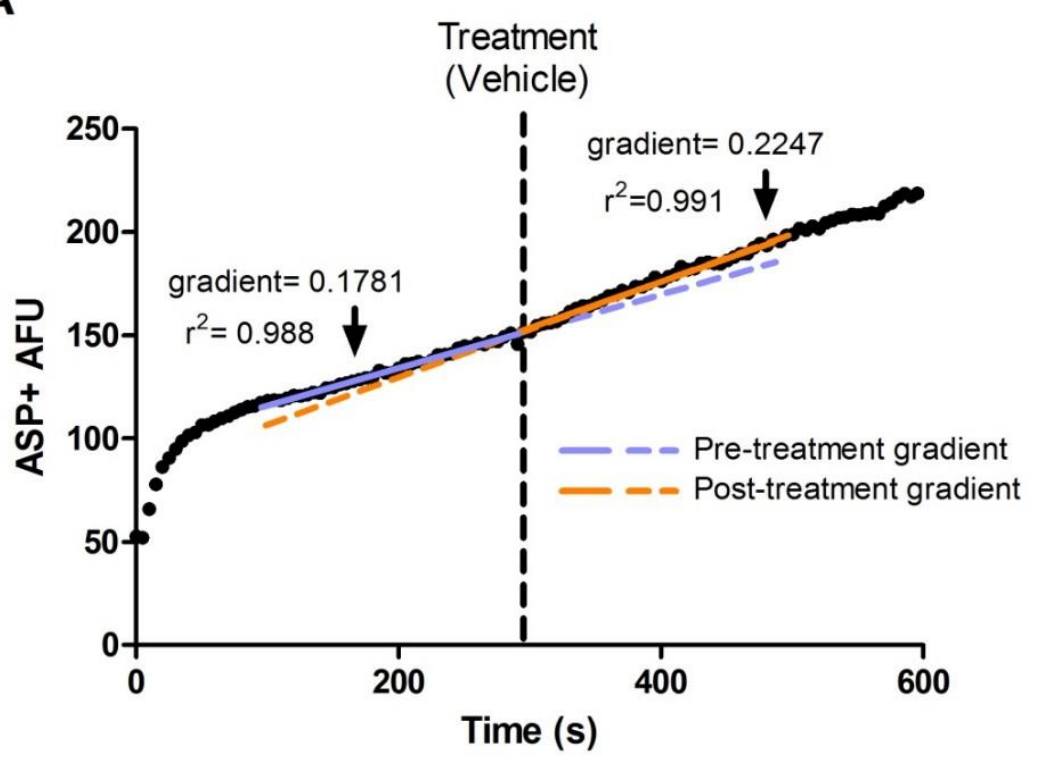

B

Treatment

$(10 \mu \mathrm{M}$ Ethy-Sal A)

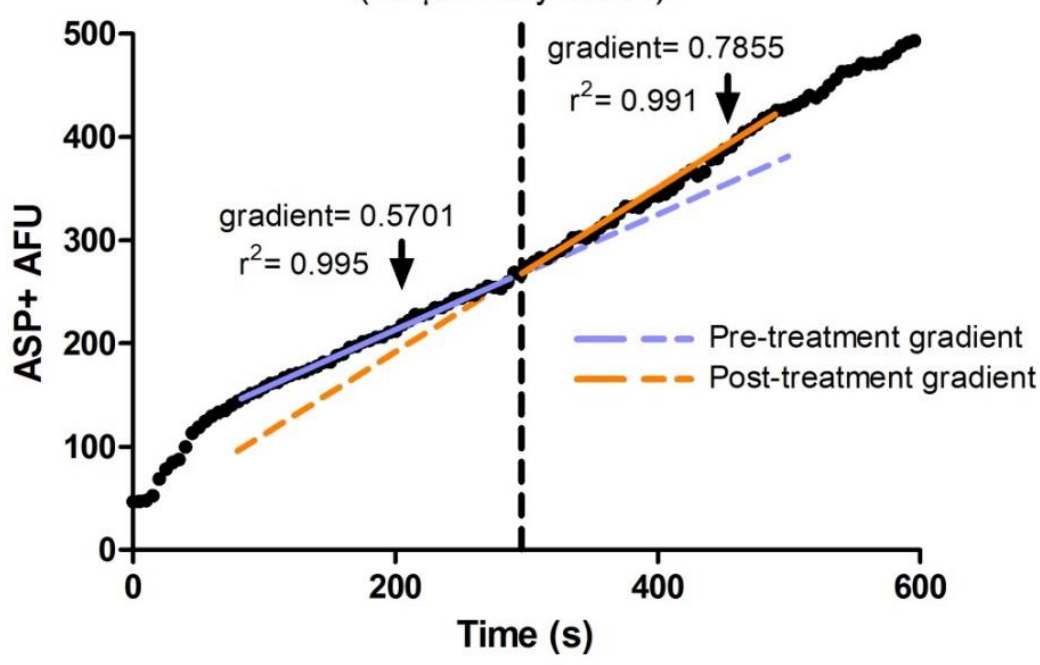

Figure 4.3. Linear ASP+ uptake in DAT/KOPr co-tranfected HEK-293 cells. Representative traces from individual YFP-hDAT-expressing cells showing increasing $\mathrm{ASP}+$ fluorescence over time with vehicle (DMSO) treatment $(\mathrm{A})$ and $\mathrm{KOPr}$ agonist treatment (B). Measurements of ASP+ fluorescence were taken every 5 seconds. ASP+ accumulation prior to treatment after the initial ASP+ binding phase (100-300 s) and after treatment (305-500 seconds) shows highly linear correlation for both vehicle and KOPr agonist treatment (Pearson correlation coefficient, $r^{2}>0.98$ ). All linear regressions are significantly non-zero. 


\subsubsection{Changes to ASP+ uptake in DAT/KOPr co-transfected HEK-293 cells after KOPr agonist treatment}

The ASP+ uptake model was used to explore the effect of KOPr activation by the novel KOPr agonist Ethy-SalA upon DAT activity in a transiently-transfected HEK-293 cellular model. YFP-hDAT expressing cells treated with $10 \mu \mathrm{M}$ of SalA showed increased ASP+ uptake of $16.9 \pm 5.05 \%$. Treatment with $1 \mu \mathrm{M}$ of Ethy-SalA increased ASP+ uptake by $11.9 \pm 4.53 \%$, while treatment with $10 \mu \mathrm{M}$ increased uptake to $31.5 \pm 6.10 \%$. Treatment with vehicle alone did not produce a significantly non-zero increase or decrease in ASP+ uptake $(-0.39 \pm 2.59 \%)$. Cells incubated with $1 \mu \mathrm{M}$ of norBNI prior to the ASP+ time-course showed a reduced ASP+ uptake of $10.5 \pm 9.01 \%$. Analysis using one-way ANOVA indicated significant differences in mean change in ASP+ uptake between treatment groups $[F(4,186)=6.53, p<0.0001]$. In comparison to vehicle, $10 \mu \mathrm{M}$ of SalA significantly increased ASP+ uptake (Dunnett's multiple comparison post-test, $p<0.05$ ) and $10 \mu \mathrm{M}$ of ESA increased ASP+ uptake with high significance $(p<0.001)$ (Fig. 4.4) 


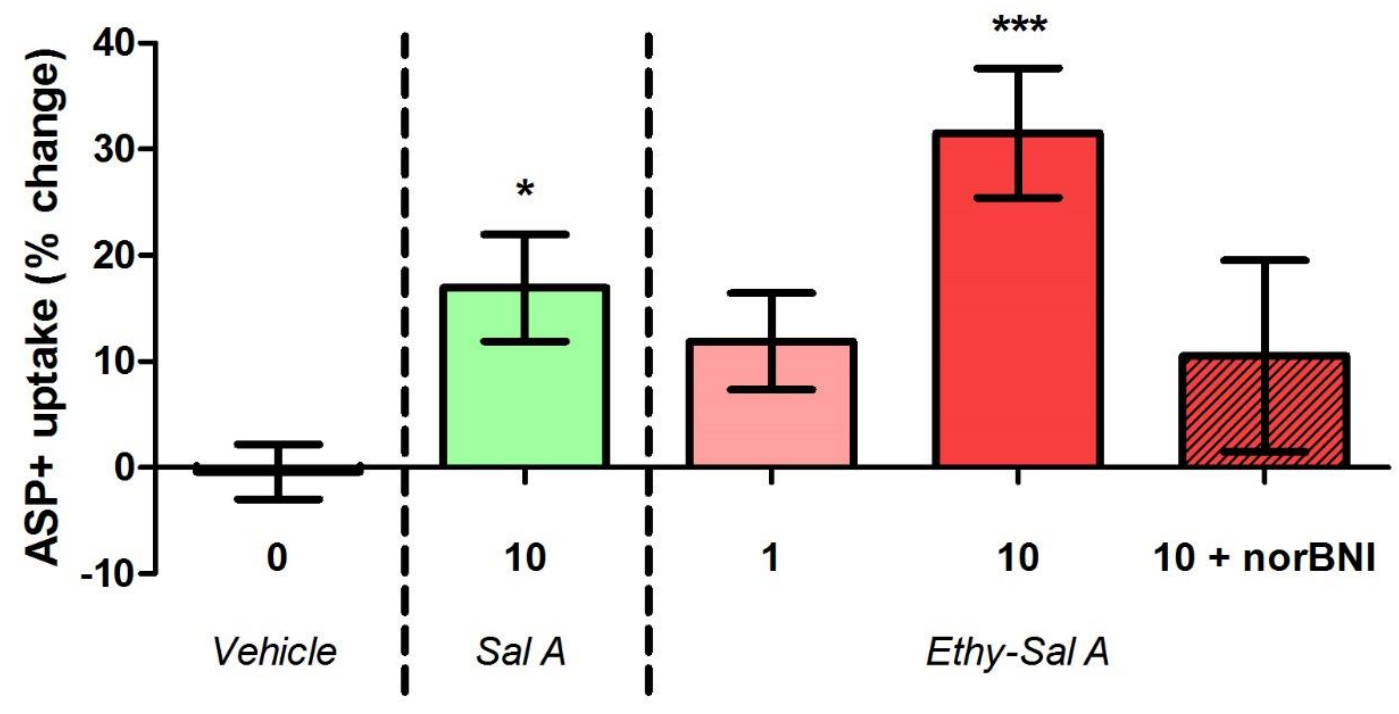

KOPr agonist concentration $(\mu \mathrm{M})$

Figure 4.4. Changes to ASP+ uptake in YFP-hDAT and myc-rKOPr co-transfected HEK-293 cells after KOPr agonist treatment. Comparisons of the slopes of linear regression lines fitted to $A S P+A F U$ values for individual cells over a $60 \mathrm{~s}$ period before and after treatment. The increase in uptake induced by treatment with SalA (10 $\mu \mathrm{M})$ and Ethy-SalA $(10 \mu \mathrm{M})$ is significantly different to the effect of vehicle treatment alone. No significant differences were detected between uptake in SalA and Ethy-SalA-treated cells. Incubation of co-transfected HEK-293 cells with $1 \mu \mathrm{M}$ of the KOPr antagonist norBNI 30 min prior to the start of the time course reduces the effect of Ethy-SalA upon ASP+ uptake. ${ }^{*}=p<0.05 ;{ }^{* * *}=p<0.001$ (one-way ANOVA, with Dunnett's post-test: comparison to vehicle). $n=26-58$ cells for vehicle, SalA and Ethy-SalA treatments; $n=12$ cells for norBNI pre-treatment. 


\subsection{Discussion}

The novel KOPr agonist Ethy-SalA has been shown to attenuate cocaine reinstatement and self-administration (Chapter 2), without sedative effects or inhibiting natural reward (Chapter 3). The cellular pathways activated by Ethy-SalA are currently un-tested. A transiently co-transfected cellular model of DAT and KOPr expression was used to explore the ability of Ethy-SalA to regulate DAT function through the KOPr, and so identify a potential mechanism for Ethy-SalA's potent anti-cocaine reward effects.

\subsubsection{YFP-hDAT and myc-rKOPr co-expression in HEK293 cells}

The HEK-293 cells used in this study replicate quickly, grow well in standard DMEM culture medium, and transfect readily (Thomas \& Smart, 2005). Originally transformed by Graham et al. (1977), HEK-293 cells have become a standard tool in cellular research. Though derived from advenoviral-transformed human embryonic kidney cells, HEK-293 cells display neuronal cell-like features, including endogenous expression of several neurofilament proteins (Shaw et al., 2002), suggesting that HEK-293 cells may be more representative of an early neuronal progenitor than a pure epithelial kidney cell . Analysis of endogenous transporter expression in HEK-293 cells has not detected significant expression of any of the monoamine transporters (DAT, SERT and norepinephrine transporters) or of opioid receptors (Ahlin et al., 2009; Thomas \& Smart, 2005 ), making the cell line ideal for transient co-transfection of KOPr and DAT.

\subsubsection{Interaction of KOPr and DAT to enhance ASP+ uptake}

ASP+, used in this study as a surrogate for dopamine, is a lipid-dependant fluorophore derived from chemical modification to the neurotoxin 1-methyl-4-phenylpyridinium (MPP+) (Magrassi et al., 1987). ASP+ shows substrate specificity for norepinephrine transporters 
(Schwartz et al., 2003), SERT (Oz et al., 2010) and DAT (Bolan et al., 2007; Zapata et al., 2007 ), and will appear to fluoresce significantly only within cells expressing these transporters. After an initial binding phase, where ASP+ fluorescence increases rapidly, $\mathrm{ASP}+$ uptake through monoamine transporters as measured by AFU within cells is highly linear (Schwartz et al., 2003), and allows the use of a within-cell experimental design, where ASP+ uptake can be plotted before and after treatment. Linear regressions of AFU traces for selected cells in this experiment had on average an $r^{2}$ value $>0.9$, indicating a strong linear correlation between increasing fluorescence and time. Comparison of initial YFP-hDAT fluorescence in selected cells and ASP+AFU at 250 seconds showed a weak positive correlation between DAT expression and the amount of internalised ASP+ (Appendix 7, Fig. 6.6). Passive ASP+ uptake into non-transfected cells is possible, due to basal expression of organic cation transporters (OCT) in Hek-293 cells (Ahlin et al., 2009); however, the relative expression levels of OCT in the HEK-293 cell line are extremely low, and are unlikely to significantly contribute to ASP+ internalisation in comparison to DAT.

Treatment with Ethy-SalA was shown to increase ASP+ uptake via KOPr activation in a dose-dependent manner, an effect which is reversed (with high variability, due to low numbers) by KOPr blockade with norBNI (Fig. 4.4). Previous studies with the classic KOPr agonists U-50,488 and SalA and Mesyl-SalA have also shown this effect in HEK-293 and neuronal-derived EM4 cells (Kivell et al., 2014 ; Simonson et al., 2015). In this assay, SalA was found to increase mean ASP+ uptake by $17 \%$, whereas a previous study identified SalA to increase uptake by approximately $45 \%$ (Simonson et al., 2015). This difference in result may be due to less successful co-transfection of KOPr with DAT in this experiment, limiting the ability of the KOPr to influence DAT activity.

The increase in ASP+ uptake seen with SalA and Ethy-SalA treatment is assumed to be due to enhanced activity of existing DAT, and not an increase in DAT cell-surface expression. Previous studies have detected no significant increases in cell-surface expression of YFP-hDAT in HEK-293 cells co-expressing rKOPr and YFP-hDAT, 60-260 
seconds after treatment with classical and novel KOPr agonists, including SalA (Simonson, PhD thesis 2011). Significant increases in YFP-hDAT expression are only observed in co-transfected HEK-293 cells after 30 min incubation with SalA (Simonson, PhD thesis 2011), which is well beyond the scope of the 5 min agonist incubation time used in the ASP+ uptake protocol. It is not yet confirmed whether Ethy-SalA treatment alters DAT or KOPr expression levels, which could be explored using Western blot and further confocal analysis of cell-surface YFP-hDAT expression over time, but it may be assumed that the agonist has similar effects to SalA and that the observed increase in ASP+ uptake over the course of the assay is due to enhanced DAT activity and not cellsurface expression.

\subsubsection{Limitations and future directions}

A high degree of variability in ASP+ uptake values was observed in this experiment (Fig. 4.4), despite consistency in the confocal settings and experimental procedure applied to each plate. Normalising AFU values to background fluorescence (i.e. non-transfected cells) for each sample could remove some of this variability.

In this confocal assay, human-derived cells (HEK-293) were transfected with fluorescentlytagged human DAT and a rat KOPr plasmid. Expression of the rat KOPr was detected in co-transfected cells, suggesting that the inter-specific difference does not affect successful transcription and translation of the receptor. The use of a rat-derived KOPr in a human cell line is problematic in terms of the validity of the model. However, the amino-acid sequence of the rat and human KOPr are very similar, with $94.1 \%$ homology reported by Simonin et al. (1995) and the online protein database UniProt (see Appendix 6). This structural homology between the two species is mirrored by functional homology in analgesic assays, and in the dysphoric effects caused by KOPr agonism in both species (Hunter et al., 1990; Reece et al., 1994 ; Walsh et al., 2001 ); though cellular data indicates that the 
rat KOPr does not show significant receptor internalisation when treated with selected KOPr agonists, whereas the human KOPr does ( $\mathrm{Li}$ et al., 1999). Repetition of this experiment with a human KOPr plasmid could confirm if any significant inter-specific differences do exist.

The use of a human cell line also limits the effective comparisons that can be made to the pre-clinical rat models used in this thesis. Analysis of DA uptake in ex vivo rat brain samples (NAc, pFC and dorsal striatal regions) using rotating-disk electrode voltammetry (RDEV) has previously been used to complement ASP+ uptake data (Kivell et al., 2014; Simonson et al., 2015), and could be used to confirm if the DAT-enhancing effects of EthySalA observed in vitro translate to a tissue model. Repetition of this confocal assay using neuronal-derived cell lines such as N2A (murine) (Klebe \& Ruddle, 1969) or NTERA-2 (human) (Andrews et al., 1984) would complement these results.

\subsubsection{Conclusions}

Treatment with Ethy-SalA $(10 \mu \mathrm{M})$ significantly increased DAT function in HEK-293 cells co-expressing YFP-hDAT and rKOPr protein, measured by up uptake of the DATspecific fluorophore ASP+. This finding suggests a cellular mechanism for the anticocaine effects of Ethy-SalA observed in self-administration studies (Chapter 2). 


\section{Chapter 5 : Discussion}

\subsection{Significance of this thesis}

The behavioural testing performed in this thesis represents the first published (Riley et al., 2014) and unpublished in vivo studies of the novel compounds Ethy-SalA and Me-SalA. Cocaine self-administration assays, hyperactivity and sensitisation assays have been used to explore the potential anti-cocaine effects of these agonists, in particular that of Ethy-SalA, with side-effect testing to understand the wider behavioural effects of these compounds. The confocal ASP+ uptake assay performed with Ethy-SalA also represents the first confirmation of this compound's mechanism of action as an indirect regulator of DAT function. The results obtained as part of this study provide a basis for further investigations of the cellular and behavioural activities of these novel compounds, in particular Ethy-SalA, with the ultimate goal of identifying a potential anti-addictive pharmacotherapy with minimal off-target effects and unfavourable side-effects.

\subsection{Summary of key findings}

5.2.1 Acute treatment with Ethy-SalA attenuates responding in cocaine selfadministration behavioural models

Acute doses of Ethy-SalA at 0.1 and $0.3 \mathrm{mg} / \mathrm{kg}$ were shown to attenuate active lever responses made by rats in a cocaine-primed reinstatement model of drug-seeking behaviour, to a level comparable to that of SalA at $0.3 \mathrm{mg} / \mathrm{kg}$. A higher dose of Ethy-SalA $(2.0 \mathrm{mg} / \mathrm{kg})$ was also shown to be sufficient to attenuate the number of infusions achieved in a progressive-ratio model of cocaine self-administration, whereas $2.0 \mathrm{mg} / \mathrm{kg}$ of SalA was not effective. These results indicate that Ethy-SalA has the ability to alter cocaineevoked drug-seeking and motivation for cocaine reward in rats, with greater potency than 
SalA. The ability of Ethy-SalA to effectively attenuate progressive-ratio cocaine administration over a minimum 2-hour session also suggests that the compound may have a longer half-life than SalA. The less-potent analogue Me-SalA was not shown to significantly alter behaviour in these models, which supports cellular data indicating that the compound acts as a less effective or more variable activator of the KOPr than SalA (Riley et al., 2014).

5.2.2 Acute Ethy-SalA treatment does not affect cocaine-induced hyperactivity or sensitisation in rats

Pre-treatment with Ethy-SalA $(0.3 \mathrm{mg} / \mathrm{kg})$ was not found to significantly reduce acute cocaine hyperactivity or supress the expression of cocaine behavioural sensitisation in rats, despite the anti-cocaine effects observed in self-administration testing. The lack of hyperactivity attenuation may be due to the low dose of agonist used, the high dose of the cocaine challenge $(20 \mathrm{mg} / \mathrm{kg})$, or to un-tested hallucinogenic effects and off-target dopaminergic effects of the compound. Cocaine sensitisation was not observed in this study, even in control animals not receiving KOPr agonist. This may be due to the low numbers used, or the confounding effects of stress, as the rats used were relocated from another facility.

\subsubsection{Low doses of Ethy-SalA and Me-SalA do not show sedative or pro-} depressive side-effects, or attenuate natural reward in rats

Ethy-SalA and Me-Sal did not attenuate the self-administration of sucrose pellets in rats when injected at a dose of $0.3 \mathrm{mg} / \mathrm{kg}$, indicating that the effective anti-cocaine reward dose of Ethy-SalA does not inhibit reward in this model of a natural reward stimulus. This effect is likely due to the more complex set of stimuli (taste, smell etc.) associated with food, which are not significantly inhibited by KOPr agonism at the dose tested. Neither Ethy- 
SalA or Me-SalA showed significant sedative effects in an open-field test of locomotor behaviour, confirming that the behavioural effect observed in cocaine-primed reinstatement testing is unlikely to be due to the sedation of test animals receiving 0.1 or $0.3 \mathrm{mg} / \mathrm{kg}$ Ethy-SalA. FST testing of acute Ethy-SalA treatment did not detect any significant pro-depressive effects when compared to vehicle treatment in rats, though these results may be confounded by the use of test animals with previous exposure to KOPr and MOR agonists, and with different experimental backgrounds. Taken together, this data suggests that the anti-reward effect of low doses of Ethy-SalA is only behaviourally significant in the presence of drugs of abuse, such as cocaine, which cause dopaminergic hyperactivity of the reward pathway.

5.2.4 Ethy-SalA treatment enhances DAT uptake of ASP+ in an in vitro cellular model

Investigations of the ability of Ethy-SalA to regulate DAT function via KOPr activation were performed using confocal microscopy. HEK-293 cells co-transfected with YFP-hDAT and rKOPr showed enhanced uptake of the fluorescent DAT substrate ASP+ when treated with $10 \mu \mathrm{M}$ of Ethy-SalA, an effect which was attenuated in cells pre-incubated with $1 \mu \mathrm{M}$ of the KOPr antagonist norBNI before Ethy-SalA treatment. This result indicates that EthySalA activation of the KOPr positively regulates DAT function in an in vitro cell model, an observation which may partially explain the anti-cocaine effects observed in selfadministration behavioural models, and supports the theory of the KOPr system as a regulator of DA within the reward pathway. The attenuation of this effect observed in cells incubated with norBNI supports the assumption that Ethy-SalA is a selective KOPr agonist, and that the cellular and behavioural effects of Ethy-SalA are mediated through the KOPr; though the high degree of error observed in norBNI-treated cells means that the result is not conclusive. 


\subsection{Limitations and future directions}

Ethy-SalA displays noticeable differences to its parent compound, SalA. Ethy-SalA is effective at a lower dose than SalA in the cocaine-primed reinstatement model $(0.1 \mathrm{mg} / \mathrm{kg}$

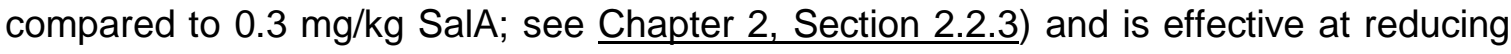
the number of infusions achieved during a progressive-ratio self-administration session (at $2.0 \mathrm{mg} / \mathrm{kg}$ ), whereas SalA is not (see Chapter 2, Section 2.2.4). Previous cellular assays by the Prisinzano laboratory have shown that Ethy-SalA is a more effective inhibitor of cAMP accumulation in a CHO KOPr-expressing model (Riley et al., 2014). Unpublished data from our laboratory also indicates that Ethy-SalA has a longer analgesic duration of action in a mouse tail-flick assay than SalA at $2.0 \mathrm{mg} / \mathrm{kg}$. Taken together, this data indicates that Ethy-SalA shows enhanced KOPr interaction compared to SalA.

There are at least two possible explanations for this effective difference: enhanced or differential binding-site interactions between Ethy-SalA and the KOPr receptor and differences in the metabolism of Ethy-Sal compared to SalA. The most current model of SalA KOPr binding (Kane et al., 2008) shows SalA binding within a pocket between KOPr transmembrane regions II and VII. Addition of different functional groups to the carbon-16 position of SalA by Riley et al. (2014) were deliberately designed to explore the role of the furan ring in the KOPr-SalA binding interaction. Addition of an ethynyl $(\mathrm{RCH})$ substituent at the carbon-16 position was shown to increase agonist potency compared to SalA $\left(\mathrm{EC}_{50}=0.019 \pm 0.004 \mathrm{nM}\right.$ compared to $\left.\mathrm{EC}_{50}=0.030 \pm 0.004 \mathrm{nM}\right)$ while addition of a methyl group $\left(\mathrm{R}-\mathrm{CH}_{3}\right.$; Me-SalA) substantially decreases potency $\left(\mathrm{EC}_{50}=0.407 \pm 0.147 \mathrm{nM}\right)$ (see Chapter 2, Fig. 2.2). This may indicate that the area of the binding pocket adjacent to the carbon-16 position of Salvinorin A is sterically hindered, as the addition of a methyl group contributes slightly more steric bulk to the molecule than the ethynyl group (see Appendix 2, Fig. 6.1). The flattened molecular geometry of the ethynyl group may allow it to fit easily within the furan-associated binding pocket, and enhance both hydrophobic and Van der 
Waals interactions, resulting in a more stable agonist-receptor association. A more complete binding of the agonist to the receptor may in turn result in enhanced or prolonged receptor activation, or even induce a functionally selective signalling profile at the KOPr. Conversely, the high variability or lack of effect seen in cocaine-primed reinstatement (Chapter 2, section 2.2.3) and progressive-ratio (Chapter 2, section 2.2.4) behavioural subjects treated with Me-SalA may be due to incomplete and sterically hindered binding of the agonist caused by the presence of the incompatible methyl group. Quantification of the binding affinities of Ethy-SalA and Me-SalA for the KOPr would confirm whether the addition of different functional groups to the carbon-16 position affects ligand-receptor interactions at the KOPr. Assays which measure the level of activation of different GPCR signalling pathways, such as comparisons of Gai and $\beta$-arrestin activity, can identify functional selective GPCR agonists (Zhou et al., 2013). Functional selectivity tests would help correlate the cellular and behavioural effects associated with Ethy-SalA treatment to an overall enhanced activation of multiple pathways, or biased activation of a few signalling pathways.

The uptake and metabolism of Ethy-SalA may also be altered compared to SalA by the addition of an ethynyl group. Steroids such as estradiol show greatly enhanced intestinal absorbance into the blood when conjugated with an ethynyl group (Reed \& Fotherby, 1979). Ethynyl groups are also known to act as inhibitors of cytochrome P450 enzymes, thereby preventing much of the metabolism of the drugs they are conjugated to (Ortiz de Montellano \& Kunze, 1980; Zhu et al., 2010). P450 enzymes are major contributors to drug metabolism (Wrighton \& Stevens, 1992), and the short metabolic half-life of SalA is likely due primarily to the activity of this family of enzymes (Teksin et al., 2009). Enhanced absorption of Ethy-SalA into the blood could help explain why significant behavioural effects are observed at a lower dose than SalA $(0.1 \mathrm{mg} / \mathrm{kg}$ Ethy-SalA compared to an effective dose of $0.3 \mathrm{mg} / \mathrm{kg} \mathrm{SalA}$ ) in a cocaine-primed reinstatement model, while P450 enzyme inhibition may explain the longevity of Ethy-SalA treatment in the progressive-ratio 
self-administration model and in the unpublished tail reflex assay. The compound MeSalA, which like SalA does not contain a metabolic inhibitory functional group, would not be afforded the same protection and longevity within the body, which may also partially explain the compound's lack of significant effect and higher variability in cocaine-primed reinstatement and progressive-ratio tests. To confirm this, metabolic time-course analyses would have to be conducted upon blood and/or urine samples of animals injected with Ethy-SalA, as well as analyses to confirm which metabolites of Ethy-SalA and Me-SalA are present and active within the CNS, at time scales correlating to the anti-cocaine effects observed in self-administration models.

Ethy-SalA and Me-SalA have been tested for activity and efficacy at the KOPr and MOR, but not for any other opioid receptors or GPCRs. SalA has previously been tested for activity at a variety of CNS receptors and found to be effectively inactive at all screened receptors other than the KOPr (Roth et al., 2002). As the novel compounds investigated in this thesis are derived from SalA and share the same target, it is unlikely that either of the compounds are significantly active at other receptors. However, it is possible that the differences in potency observed between Ethy-SalA and SalA and the inability of EthySalA to significantly attenuate cocaine hyperactivity and sensitisation effects may be explained by the interaction of Ethy-SalA with other signalling systems within the brain. A wide activity screen of Ethy-SalA against key CNS receptors, including delta-opioid, 5-HT, GABA and glutamate receptors, would validate its current designation as a selective KOPr agonist.

This thesis considers only the effects of acute KOPr agonist administration. To widen understanding of these novel KOPr agonists, the effects of chronic KOPr administration will have to be taken into consideration, particularly as chronic KOPr activation with classical agonists has been shown to promote drug-seeking and addictive-like behaviours (Negus, 2004). The effects of acute Ethy-SalA treatment should also be observed at longer time scales ( $>2$ hours after treatment) in anti-cocaine and side effect testing, as recent 
research indicates that acute SalA $(2.0 \mathrm{mg} . \mathrm{kg})$ continues to show significant effects up to 24 hours after treatment (Chartoff et al., 2015).

The separation of behavioural and cell-specific testing hinders direct comparison between animal and cellular models, and the explanation of the mechanisms of behavioural effects. Recent optogenetic advances have allowed precision targeting and activation of selected brain areas, through a combination of directed adenoviral transfection of light-sensitive ion channels and the insertion of micro-LED devices (Kim et al., 2013; Siuda et al., 2015). This technique has already been used to show the existence of sub-population of KOPrexpressing neurons in the NAc with opposing behavioural effects (Al-Hasani et al., 2015). Cellular manipulations of an in vivo model has huge advantages over whole-body drug administration, and will be a useful method for determining the effects of KOPr activation in isolated regions of the brain, in order to better understand the full complexity of the KOPr system.

Finally, this investigation of the effects of novel KOPr agonists is greatly limited by confinement to a single mammalian model and a single cell line. Self-administration models using non-human primates have already been used to test the ability of SalA to alter drug-seeking behaviour, and would be a useful next step in the pre-clinical testing of Ethy-SalA and other novel KOPr agonists. The use of HEK-293 cells in this investigation also limits the conclusions that can be drawn from the data obtained. Performing the assay using a variety of cultured cell types, particularly cells of neural lineage, would confirm the regulatory effect of the KOPr upon DAT this effect would be observed in a range of different cell types.

The development of novel SalA-derived anti-addictive medications shows most promise with regards to the prevention of prescription-opioid abuse. Self-administration studies in rhesus monkeys have identified that co-infusion of SalA with the opiates remifentanil and oxycodone can reduce the reinforcing and rewarding qualities of the drugs. Addition of 
KOPr agonists to existing opioids could reduce their addictive potential, allowing patients to receive effective pain relief without the development of dependence upon the drug.

\subsection{Final conclusions}

This thesis explores the behavioural and cellular effects of Ethy-SalA, a potent novel KOPr agonist, and a less potent agonist Me-SalA. Ethy-SalA shows anti-cocaine effects in selfadministration models of relapse and reward motivation in Sprague-Dawley rats, but does not significantly affect cocaine hyperactivity or the expression of behavioural sensitisation to cocaine in locomotor assays, in contrast to previously-tested KOPr agonists. Neither Ethy-SalA or Me-SalA show significant sedative side-effects at low doses, and also do not attenuate the self-administration of the natural food reward, sucrose. Ethy-SalA also does not display any pro-depressive side-effects in the Forced Swim Test, a model of depression-like behaviour. The anti-cocaine effects observed in self-administration studies are at least in part due to KOPr-mediated enhancement of DAT activity, as enhanced uptake of the DAT substrate ASP+ is observed in DAT/KOPr co-transfected HEK-293 cells treated with Ethy-SalA. Further behavioural and cellular testing of Ethy-SalA will establish whether the novel compound shows promise as a lead for the development of an antiaddictive pharmacotherapy. 


\section{Chapter 6 : Supplementary information}

Appendix 1: Buffer solutions

Phosphate Buffered Saline (PBS) pH 7.4 (10x concentrate)

$$
\text { Concentration (M) (g/L) }
$$

$\mathrm{NaCl}$

$1.4 \mathrm{M}$

80

$\mathrm{KCl}$

$26.8 \mathrm{mM}$

2.0

$\mathrm{Na}_{2} \mathrm{HPO}_{4}$

$81.0 \mathrm{mM}$

26.8

$\mathrm{KH}_{2} \mathrm{PO}_{4}$

$14.7 \mathrm{mM}$

2.4

KREBS Buffer pH 7.4

Concentration (M) (g/L)

$\mathrm{NaCl}$

$130 \mathrm{mM}$

7.6

$\mathrm{KCl}$

$1.3 \mathrm{mM}$

0.10

$\mathrm{CaCl}_{2}$

$2.2 \mathrm{mM}$

0.24

$\mathrm{MgSO}_{4} \cdot 6 \mathrm{H}_{2} \mathrm{O}$

$1.2 \mathrm{mM}$

0.27

$\mathrm{KH}_{2} \mathrm{PO}_{4}$

$1.2 \mathrm{mM}$

0.16

HEPES

$10 \mathrm{mM}$

2.6

D-Glucose

$10 \mathrm{mM}$

1.8 


\section{Appendix 2: In silico modelling of novel KOPr agonists}

Chemical drawing software (MarvinSketch ${ }^{\mathrm{TM}}$ ) was used to construct representations of the molecules SalA, Ethy-SalA and Me-SalA as 3-dimensional 'ball-and-stick' models using MarvinSpace 3-D visualisation software (ChemAxon software, Budapest, Hungary). Balland-stick structures were overlaid with Van der Waals surface projections of each molecule, to visualise differences in electron density around the carbon-16 position of each molecule. The carbons of the SalA structural backbone were numbered as per Ortega et al. (1982).

Van der Waals surface modelling of the three compounds SalA, Ethy-SalA and Me-SalA identified areas of steric difference around the carbon-16 position of each molecule. Both Ethy-SalA and Me-SalA display increased areas of steric bulk at the carbon-16 position in comparison to SalA, due to the presence of the respective ethynyl and methyl groups. 


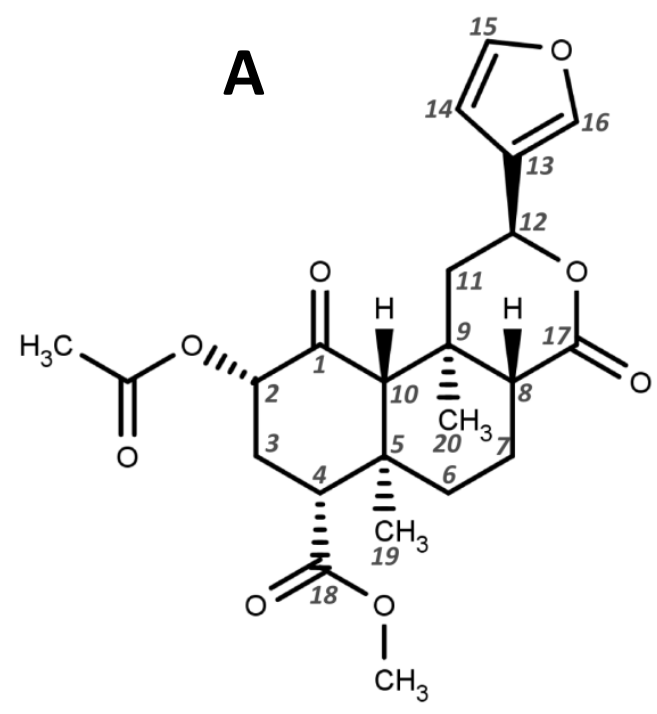

B

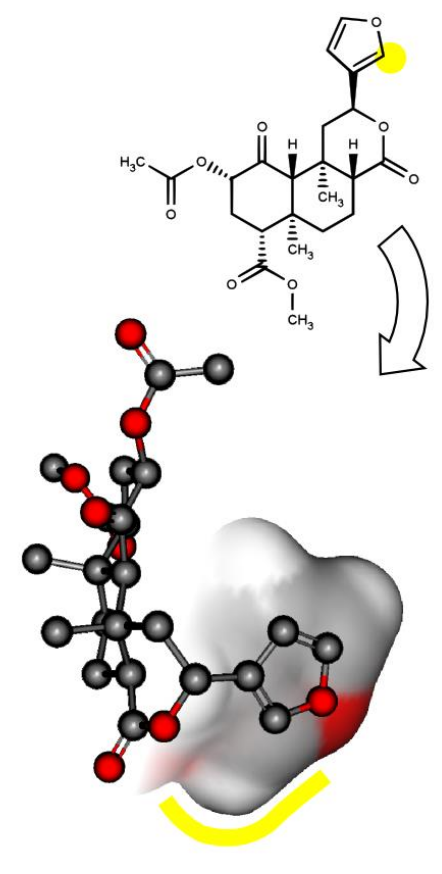

C

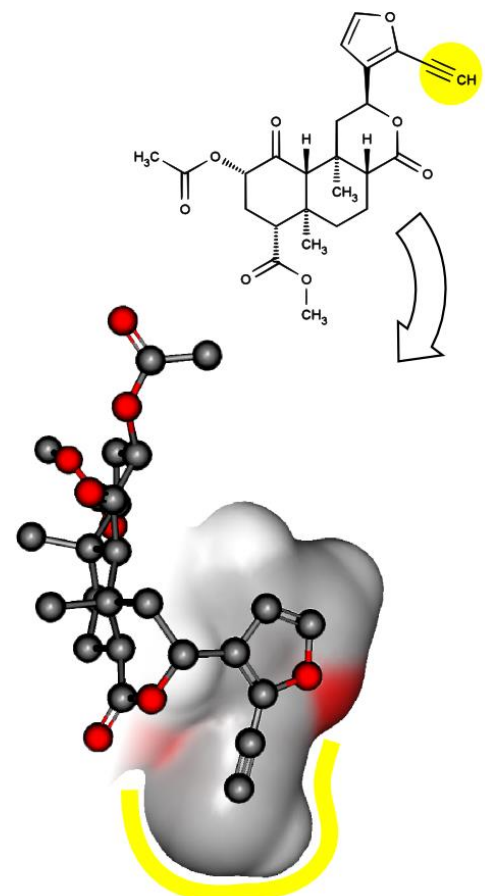

D

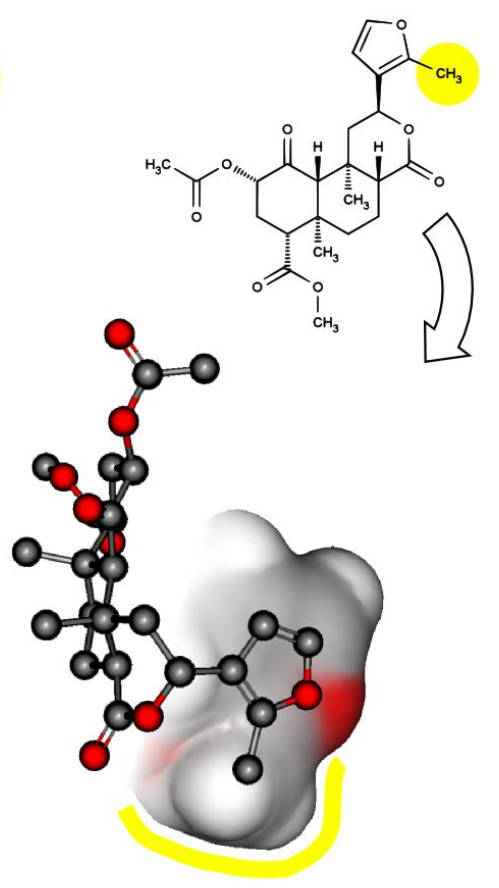

David Young 2014

Figure 6.1. Computer-generated modelling of KOPr compounds. (A) Carbon atom numbering of the SalA structure, as originally determined by Ortega et al. (1982). Van der Waal's surface-area models were generated From the chemical structures of SalA (B), 16 ethynyl SalA (C), and 16-methyl SalA (D), with overlaid ball-and-stick models representing the molecular backbone (hydrogens omitted; carbon= grey, oxygen= red). Steric areas associated with the carbon-16 position are highlighted in yellow. Both Ethy-SalA and MeSalA show increased steric bulk at the carbon-16 position in comparison to SalA. While the ethynyl group of $C$ is longer than the methyl group of $D$, it displays an overall decreased steric bulk compared to the methyl group [images generated using MarvinSketch and MarvinSpace chemical drawing software]. 


\section{Appendix 3.1: Catheter preparation for surgery}

Catheters for use in self-administration were prepared at least 2 days before surgery to allow the silicon coating to completely dry before use. 22-gauge BD needles (Becton Dickinson Ltd., NZ Auckland, New Zealand) were cut and blunted with a hand-held rotary sander (Dremel 3000 ) to a length of approximately $3 \mathrm{~cm}$, and rinsed thoroughly in $70 \%$ ethanol to remove any particulate matter from the gauge. This metal piece was inserted into a section of silastic tubing ( $0.5 \times 0.9 \mathrm{~mm} \mathrm{ID,C-P96115-02),} \mathrm{so} \mathrm{that} \mathrm{roughly} \mathrm{half} \mathrm{the}$ metal was covered. The catheter was flushed with $70 \%$ ethanol and allowed to dry before being coated with a thin, smooth layer of silicon. A minimum of two such coats were applied, with each coat being allowed a full day to dry before application of the next. After the final coating, a small nub of silicon was added to the catheter at the opposite end to the metal piece to act as an anchor for the catheter once inside the vein. The tubing was cut at an angle approximately $4 \mathrm{~cm}$ from the silicon nub to allow for insertion of the catheter into the vein (see Fig. 6.2).

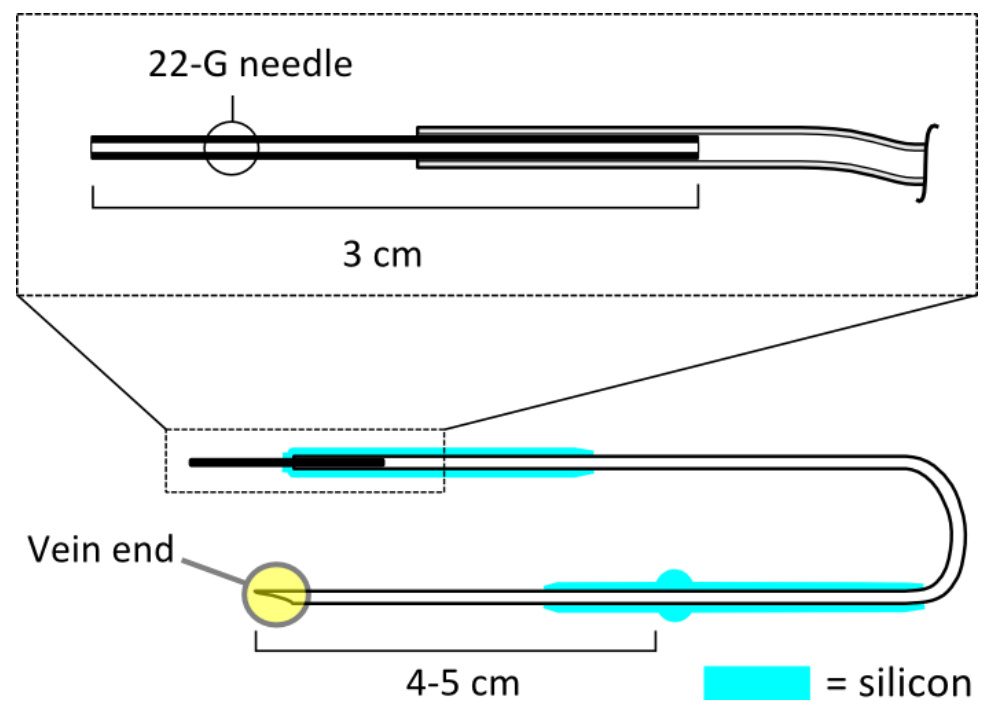

Figure 6.2. Intra-jugular catheter. Schematic of the catheter used in self-administration surgery. Total length of catheter $=$ approx. $20 \mathrm{~cm}$ long. Materials are silastic tubing $(0.5 \mathrm{x}$ 0.9 mm ID, C-P96115-02), 22-Gauge BD needles, and silicon (Selleys Wet-Area Silicone Sealant). 


\section{Appendix 3.2: Intra-jugular catheter surgery}

Surgery was performed upon animals undergoing cocaine self-administration. Before surgery, rats weighing $300-350 \mathrm{~g}$ were handled for two days. On the day of surgery, rats were anaesthetised with an intraperitoneal (i.p.) injection of a 2:1 mixture of ketamine and xylazine (90 and 9 mg/kg; Provet NZ Pty. Ltd., Auckland, New Zealand). The rat was considered to be appropriately anaesthetised upon complete loss of muscle tone and lack of a kicking reflex when a strong pinching stimulus was applied to the hind footpad. The crown of the head and the right-hand side of the chest were shaved, and the skin swabbed with Vetadine (1.6\% iodine w/v: Provet, New Zealand) and 70\% ethanol. Lacrilube (Provet, New Zealand) was applied to the eyes to reduce dryness over the course of the surgery. A subcutaneous (s.c.) injection of the painkiller carprofen (Provet, New Zealand) at 5 $\mathrm{mg} / \mathrm{kg}$ was given directly prior to the start of the surgical procedure. A small incision directly above the right jugular vein was made through the skin of the chest, and the underlying tissue gently teased apart to expose the vein. Once located, the vein was securely tied off using suture wire at the head end of the vein. A small hole was then cut through the skin at the back of the head, and the bevelled end of the catheter run subcutaneously around the neck to the exposed jugular vein. A small incision was made part-way through the jugular, and the end of the catheter passed into the vein to a depth of around $4 \mathrm{~cm}$. The catheter was then tied securely into the vein, using the silicon nub as an anchor. Catheter patency was confirmed by flushing the catheter with 3 unit/mL heparin solution in a $1 \mathrm{~mL}$ syringe attached to the metal piece and drawing a little blood back into the tubing. The open end of the metal piece was then capped with a short section of close-ended tubing. A long sagittal cut was made into the skin of the head and the underlying tissue carefully scraped away to expose the skull. The surface was rubbed with terramycin powder to dry the bone and to stem any bleeding. The head of the rat was then securely placed in a restraining device and four small holes were drilled into the skull. Jeweller's screws were threaded into each of the holes, leaving a $2-3 \mathrm{~mm}$ gap between the head of the screw and 
the skull. The metal of the catheter was placed running sagittally between these screws and a section of the metal bent upwards at an angle of between $45-90^{\circ}$ from the skull. Ostron powder and Ostron liquid (Henry Schein Shalfoon, Auckland, New Zealand) was mixed to form a slurry and was placed over the exposed portion of the skull to secure the catheter. Using more Ostron mixture, a larger screw with the thread pointing upwards was placed on the head just behind the protruding metal piece. The Ostron was then left to dry, forming a hard acrylic-resin headpiece. Chest and head wounds were sealed with small amounts of superglue and dusted with terramycin powder. $10 \mathrm{~mL}$ of sodium lactate solution (Provet, New Zealand) was injected s.c. into the flank on either side of the rat (5 $\mathrm{mL}$ each side) to replace electrolytes lost over the course of the surgery.

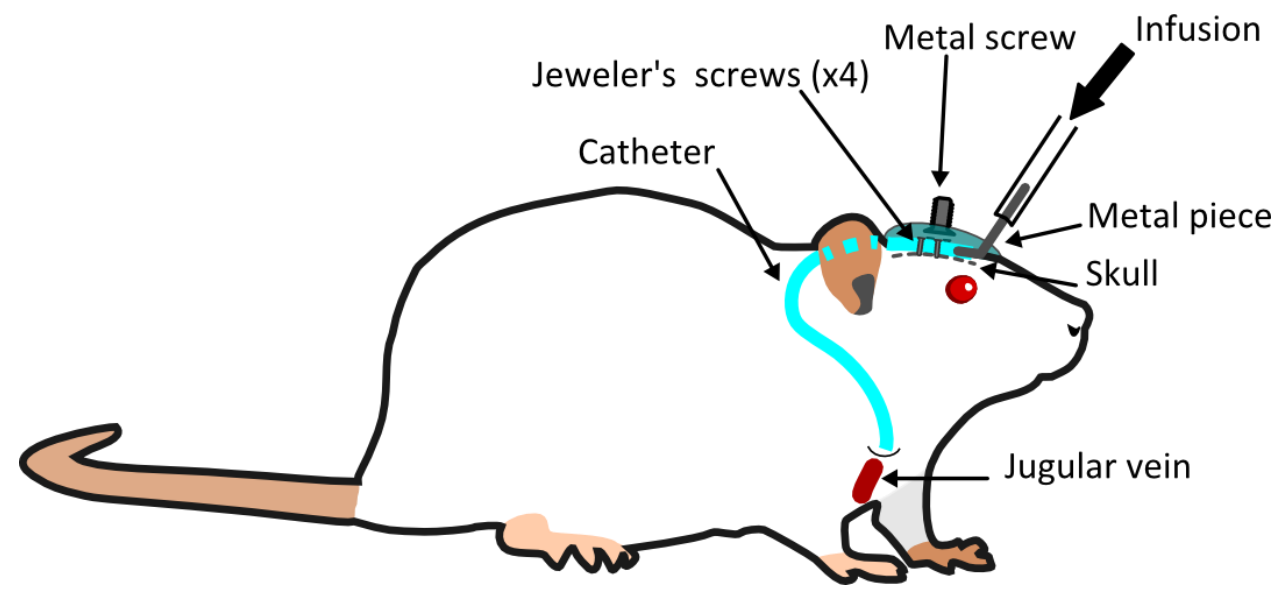

Figure 6.3. Catheter placement. Schematic of catheter and headpiece placement for intravenous self-administration. 


\section{Appendix 3.3: Post-operative care}

All rats undergoing jugular surgery were allowed a minimum of five days to recover, with weights carefully monitored over the recovery period. Carprofen at $5 \mathrm{mg} / \mathrm{kg}$ was also given s.c. for two days post-surgery. Catheters were flushed daily with $0.2 \mathrm{~mL}$ of a sterile solution of $30 \mathrm{unit} / \mathrm{mL}$ heparin and $100,000 \mathrm{unit} / \mathrm{mL}$ penicillin (Provet, New Zealand) to prevent infection and the formation of blood clots within the tubing. Catheters were also tested weekly for patency, by using a $1 \mathrm{~mL}$ BD syringe with a plastic tubing attachment to flush the catheter with $0.1 \mathrm{~mL}$ of heparin/penicillin solution and then draw back approximately 0.1-0.2 $\mathrm{mL}$ of liquid. If blood appeared in the tubing, the line was considered patent. If this failed, a secondary method of testing was to flush the catheter with $0.15 \mathrm{~mL}$ of a $50 \mathrm{mg} / \mathrm{mL}$ pentobarbital solution, and observe the rat for immediate loss of muscle tone. If this occurred, the catheter was considered patent; if not, it was assumed that the catheter had developed a leak, and the rat was operated on to insert a second catheter into the left jugular. After failure of a second catheter, the rat was no longer viable for experimentation. If the catheter developed a block due to clot formation within the metal piece, a replacement metal piece was inserted into the existing catheter and attached to the headpiece, under general anaesthesia. 


\section{Appendix 4: Latin square treatment designs (self-admin)}

For experiments where subjects received multiple treatments, a latin square design was to counterbalance treatments and to control for the potentially confounding effect of a treatment order upon the results obtained.

\begin{tabular}{|c|c|c|c|c|c|c|c|}
\hline & \multicolumn{6}{|c|}{ Treatment order } \\
\hline & & A & $B$ & $\mathrm{C}$ & $\mathrm{D}$ & $E$ & $\mathrm{~F}$ \\
\hline \multirow{12}{*}{ 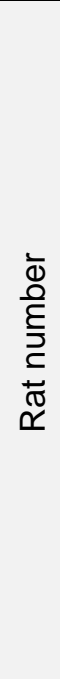 } & 1 & SalA & $0.3 \mathrm{MeSA}$ & 1.0 MeSA & Veh & $0.1 \mathrm{ESA}$ & 0.3 ESA \\
\hline & 2 & $0.1 \mathrm{ESA}$ & SalA & $0.3 \mathrm{MeSA}$ & $1.0 \mathrm{MeSA}$ & Veh & 0.3 ESA \\
\hline & 3 & $0.3 \mathrm{ESA}$ & $0.1 \mathrm{ESA}$ & SalA & $0.3 \mathrm{MeSA}$ & 1.0 MeSA & Veh \\
\hline & 4 & Veh & $0.3 \mathrm{ESA}$ & $0.1 \mathrm{ESA}$ & SalA & 1.0 MeSA & $0.3 \mathrm{MeSA}$ \\
\hline & 5 & 1.0 MeSA & Veh & 0.3 ESA & $0.1 \mathrm{ESA}$ & SalA & $0.3 \mathrm{MeSA}$ \\
\hline & 6 & $0.3 \mathrm{MeSA}$ & 1.0 MeSA & Veh & $0.3 \mathrm{ESA}$ & $0.1 \mathrm{ESA}$ & SalA \\
\hline & 7 & Veh & 1.0 MeSA & $0.3 \mathrm{MeSA}$ & SalA & $0.1 \mathrm{ESA}$ & 0.3 ESA \\
\hline & 8 & $0.3 \mathrm{ESA}$ & Veh & 1.0 MeSA & $0.3 \mathrm{MeSA}$ & SalA & $0.1 \mathrm{ESA}$ \\
\hline & 9 & $0.1 \mathrm{ESA}$ & $0.3 \mathrm{ESA}$ & Veh & $0.3 \mathrm{MeSA}$ & 1.0 MeSA & SalA \\
\hline & 10 & SalA & $0.3 \mathrm{ESA}$ & $0.1 \mathrm{ESA}$ & Veh & 1.0 MeSA & $0.3 \mathrm{MeSA}$ \\
\hline & 11 & $0.3 \mathrm{MeSA}$ & SalA & $0.1 \mathrm{ESA}$ & $0.3 \mathrm{ESA}$ & Veh & $1.0 \mathrm{MeSA}$ \\
\hline & 12 & 1.0 MeSA & $0.3 \mathrm{MeSA}$ & SalA & $0.1 \mathrm{ESA}$ & 0.3 ESA & Veh \\
\hline
\end{tabular}

Table 6.1. Cocaine reinstatement treatments. Latin square design used in cocaine selfadministration $\mathrm{KOPr}$ treatment. MeSA $=$ Me-SalA; ESA $=$ Ethy-SalA; Veh= vehicle. Numbers represent the doses of treatments (e.g. $0.3=0.3 \mathrm{mg} / \mathrm{kg}$ ). SalA was given at a dosage of $0.3 \mathrm{mg} / \mathrm{kg}$. 


\begin{tabular}{|c|c|c|c|c|c|c|c|c|c|}
\hline & \multicolumn{8}{|c|}{ Treatment order } \\
\hline & & $A$ & B & C & $D$ & $E$ & $\mathrm{~F}$ & $\mathrm{G}$ & $\mathrm{H}$ \\
\hline \multirow{8}{*}{ 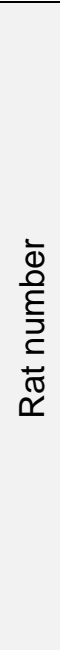 } & 1 & $0.3 \mathrm{ESA}$ & Veh & U69 & $\begin{array}{c}1.0 \\
\text { MeSA }\end{array}$ & 1.0 ESA & $\begin{array}{c}2.0 \\
\text { MeSA }\end{array}$ & SalA & $\begin{array}{c}2.0 \\
\text { ESA }\end{array}$ \\
\hline & 2 & $\begin{array}{c}2.0 \\
\text { MeSA }\end{array}$ & $0.3 \mathrm{ESA}$ & Veh & U69 & $\begin{array}{c}1.0 \\
\text { MeSA }\end{array}$ & $1.0 \mathrm{ESA}$ & $\begin{array}{c}2.0 \\
\text { ESA }\end{array}$ & SalA \\
\hline & 3 & 1.0 ESA & $\begin{array}{c}2.0 \\
\text { MeSA }\end{array}$ & 0.3 ESA & Veh & U69 & $\begin{array}{c}1.0 \\
\text { MeSA }\end{array}$ & SalA & $\begin{array}{c}2.0 \\
\text { ESA }\end{array}$ \\
\hline & 4 & $\begin{array}{c}1.0 \\
\text { MeSA }\end{array}$ & $1.0 \mathrm{ESA}$ & $\begin{array}{c}2.0 \\
\text { MeSA }\end{array}$ & $0.3 \mathrm{ESA}$ & Veh & U69 & $\begin{array}{c}2.0 \\
\text { ESA }\end{array}$ & SalA \\
\hline & 5 & $0.3 \mathrm{ESA}$ & 1.0 ESA & $\begin{array}{c}1.0 \\
\text { MeSA }\end{array}$ & $\begin{array}{c}2.0 \\
\text { MeSA }\end{array}$ & U69 & Veh & SalA & $\begin{array}{c}2.0 \\
\text { ESA }\end{array}$ \\
\hline & 6 & $\begin{array}{c}2.0 \\
\mathrm{MeSA}\end{array}$ & U69 & Veh & 0.3 ESA & $1.0 \mathrm{ESA}$ & $\begin{array}{c}1.0 \\
\text { MeSA }\end{array}$ & $\begin{array}{c}2.0 \\
\text { ESA }\end{array}$ & SalA \\
\hline & 7 & $\begin{array}{c}1.0 \\
\text { MeSA }\end{array}$ & $\begin{array}{c}2.0 \\
\text { MeSA }\end{array}$ & U69 & Veh & $0.3 \mathrm{ESA}$ & $1.0 \mathrm{ESA}$ & SalA & $\begin{array}{c}2.0 \\
\text { ESA }\end{array}$ \\
\hline & 8 & $1.0 \mathrm{ESA}$ & $\begin{array}{c}1.0 \\
\mathrm{MeSA}\end{array}$ & $\begin{array}{c}2.0 \\
\text { MeSA }\end{array}$ & U69 & Veh & $0.3 \mathrm{ESA}$ & $\begin{array}{c}2.0 \\
\text { ESA }\end{array}$ & SalA \\
\hline
\end{tabular}

Table 6.2. Progressive-ratio treatments. Latin square design used for progressive-ratio KOPr treatments. MeSA $=$ Me-SalA, ESA $=$ Ethy-SalA, U69= U-69,593, U50=U-50,488, $\mathrm{Veh}=$ vehicle. SalA and Ethy-SalA treatments at $2.0 \mathrm{mg} / \mathrm{kg}$ were added to the design retrospectively in order to test the higher doses.

\begin{tabular}{|c|c|c|c|c|c|c|}
\hline & \multicolumn{5}{|c|}{ Treatment order } \\
\hline & & A & B & C & D & $E$ \\
\hline \multirow{7}{*}{ 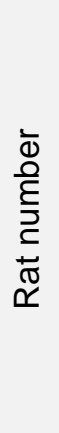 } & 1 & Veh & U69 & MeSA & ESA & U50 \\
\hline & 2 & U69 & MeSA & ESA & U50 & Veh \\
\hline & 3 & MeSA & ESA & U50 & Veh & U69 \\
\hline & 4 & ESA & U69 & Veh & U50 & MeSA \\
\hline & 5 & U69 & Veh & U50 & MeSA & ESA \\
\hline & 6 & Veh & U50 & U69 & MeSA & ESA \\
\hline & 7 & U50 & MeSA & ESA & U69 & Veh \\
\hline
\end{tabular}

Table 6.3. Sucrose self-administration treatments. Latin square design for sucrose administration $\mathrm{KOPr}$ treatments. MeSA $=\mathrm{Me}-\mathrm{Sal} A(0.3 \mathrm{mg} / \mathrm{kg}), \mathrm{ESA}=$ Ethy-SalA $(0.3$ $\mathrm{mg} / \mathrm{kg}), U 69=U-69,593(0.3 \mathrm{mg} / \mathrm{kg}), U 50=U-50,488,(10 \mathrm{mg} / \mathrm{kg}), \mathrm{Veh}=$ vehicle 


\section{Appendix 5: Cocaine hyperactivity with SalA pre-treatment}

A

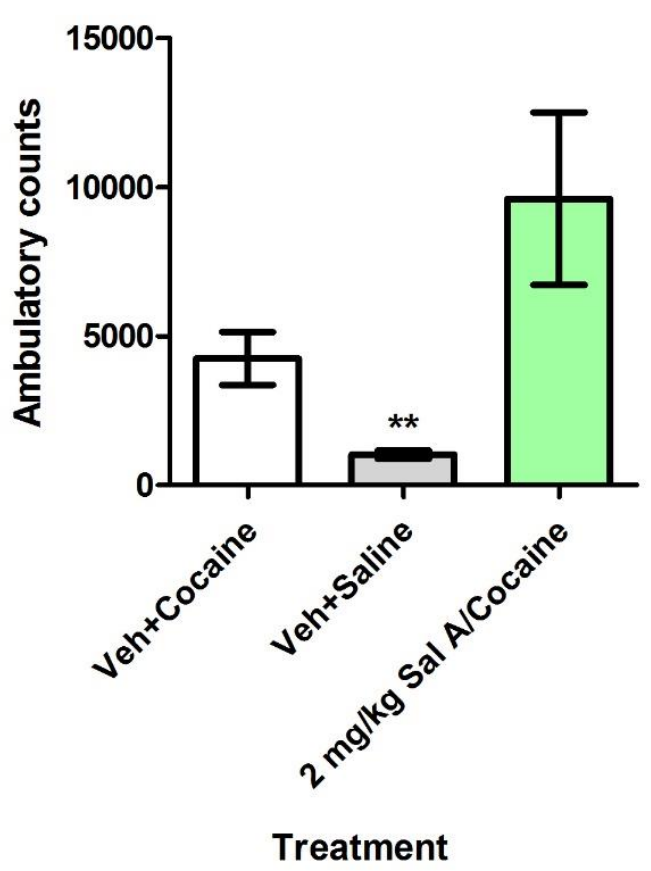

B

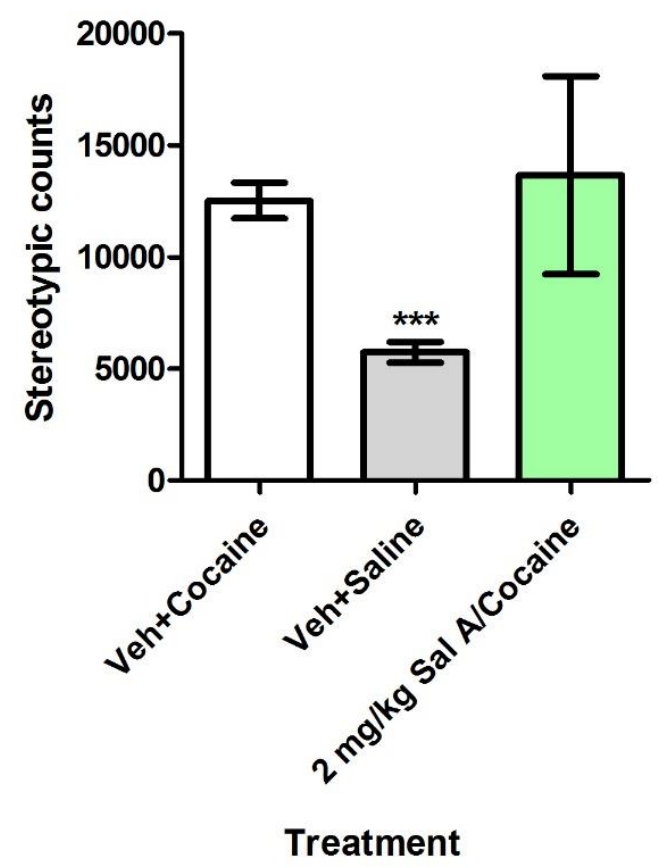

Figure 6.4. SalA cocaine hyperactivity. Cocaine hyperactivity data for two animals pretreated with $2.0 \mathrm{mg} / \mathrm{kg}$ SalA before a $20 \mathrm{mg} / \mathrm{kg}$ injection of cocaine, compared to animals receiving vehicle pre-treatment before either $20 \mathrm{mg} / \mathrm{kg}$ cocaine or saline injections. Analysis of total ambulatory counts $(A)$ identified a significant difference between means $[F(2,22)=7.90, p<0.05]$, with vehicle-saline and $2.0 \mathrm{mg} / \mathrm{kg}$ SalA-cocaine treatments showing significant differences to the hyperactivity negative control. Analysis of stereotypic activity counts (B) also identified significant differences between the means $[F(2,22)=15.30, p<0.0001]$, with the vehicle-saline treatment group showing a significant difference to the hyperactivity negative control. ${ }^{*}=p<0.05,{ }^{* * *} p<0.001$; Kruskal-Wallis test (non-parametric) with Dunn's multiple comparison post-test. $n=2-15$. 


\section{Appendix 6: Rat/Human KOPr homology}

Rat and human KOPr sequences as listed on UniProt (http://www.uniprot.org/) were searched and aligned using UniProt software. Analysis identified 94.1\% homology between human and rat KOPr amino-acid sequences. Screenshots of the alignment output (dated 20 November 2015) are shown below.

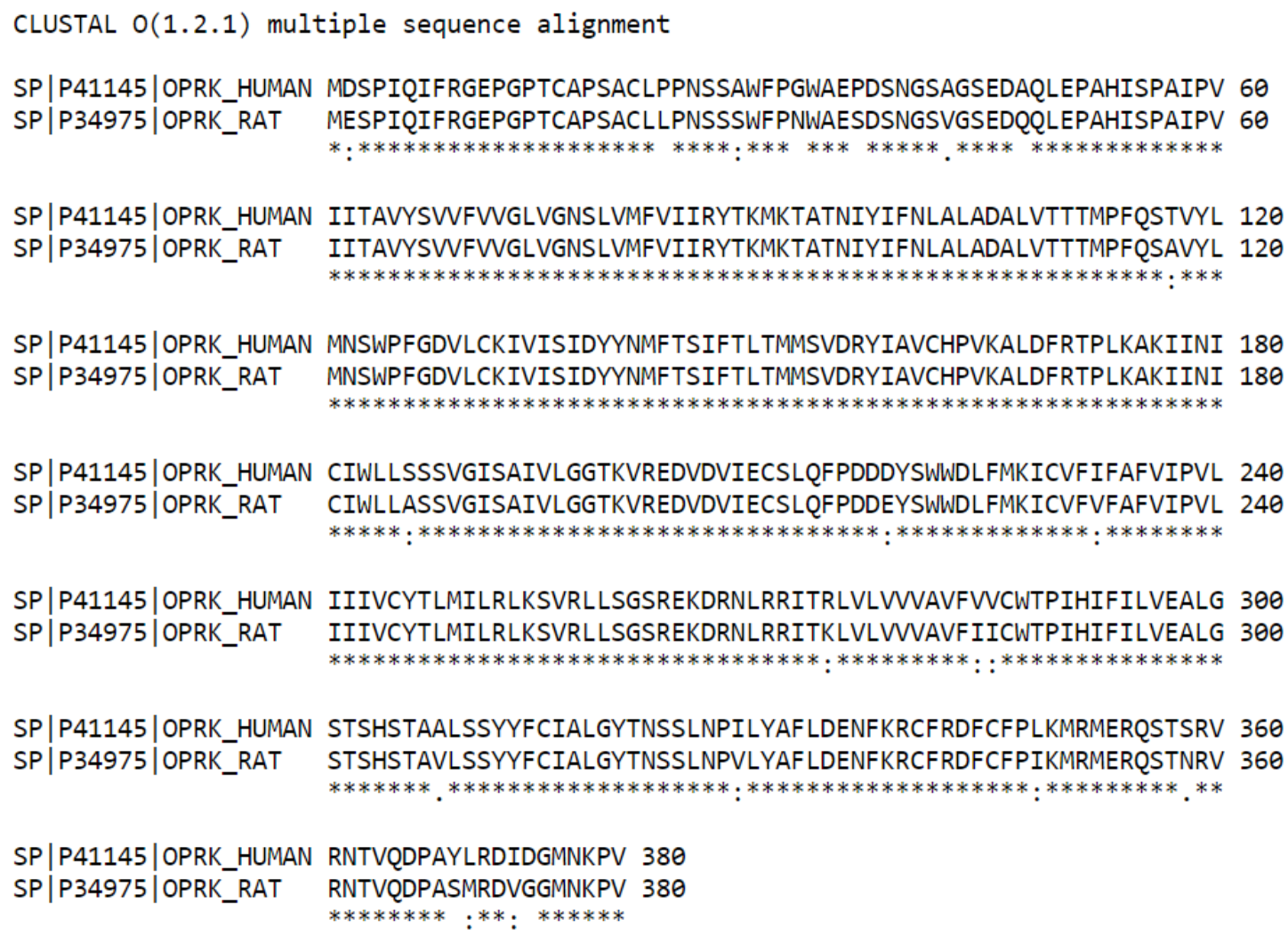
alignment engine. The algorithm is described in Söding, J. (2005) 'Protein homology detection by HMM-HMM comparison'. Bioinformatics 21, 951-960. 
Appendix 7: ASP+ accumulation and YFP-hDAT expression

The amount of YFP-hDAT expression (approximated by measures of AFU made using the EYFP confocal microscope filter at time $=0$ seconds) is weakly positively correlated [Pearson correlation coefficient, $\left.r^{2}=0.301\right]$ with the level of ASP + accumulation within cells at 250 (approximated by AFU measured with the ASP + confocal microscope filter at $t=250$ s). The correlation between YFP-hDAT expression and ASP+ accumulation was determined using linear regression modelling.

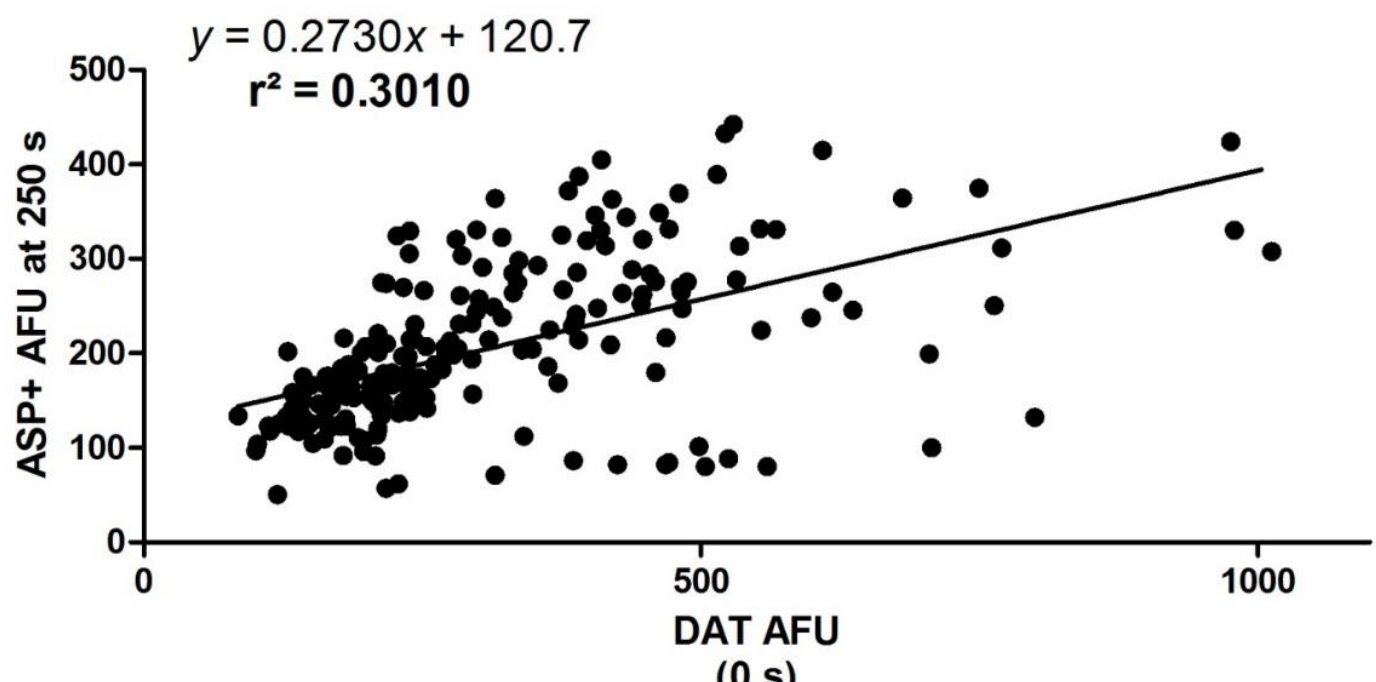

Figure 6.5. Correlation of YFP-hDAT expression and cellular ASP+ accumulation in untreated transfected HEK-293 cells. Comparison of YFP-hDAT AFU at time $=0 \mathrm{~s}$ and ASP + AFU at time $=250 \mathrm{~s}$ for individual cells. The level of YFP-hDAT expression is weakly positively correlated with ASP+ AFU accumulation (Pearson correlation coefficient, $\mathrm{r}^{2}=$ $0.301)$. The linear regression is significantly non-zero $(p<0.0001) . n=221$ cells. 


\section{References}

Adams, L. M., \& Geyer, M. A. (1985). A proposed animal model for hallucinogens based on LSD's effects on patterns of exploration in rats. Behavioral Neuroscience, 99(5), 881-900. doi: http://dx.doi.org/10.1037/0735-7044.99.5.881

Ahlin, G., Hilgendorf, C., Karlsson, J., Szigyarto, C. A.-K., Uhlén, M., \& Artursson, P. (2009). Endogenous Gene and Protein Expression of Drug-Transporting Proteins in Cell Lines Routinely Used in Drug Discovery Programs. Drug Metabolism and Disposition, 37(12), 2275-2283. doi: 10.1124/dmd.109.028654

Ahmed, S. H. (2005). Imbalance between drug and non-drug reward availability: A major risk factor for addiction. Eur J Pharmacol, 526(1-3), 9-20. doi: http://dx.doi.org/10.1016/j.ejphar.2005.09.036

Ahmed, S. H. (2012). The Science of Making Drug-Addicted Animals. Neuroscience, 211, 107-125. doi: DOI 10.1016/j.neuroscience.2011.08.014

Ahmed, S. H., \& Koob, G. F. (1998). Transition from moderate to excessive drug intake: change in hedonic set point. Science (New York, N.Y.), 282(5387), 298-300.

Al-Hasani, R., McCall, Jordan G., Shin, G., Gomez, Adrian M., Schmitz, Gavin P., Bernardi, Julio M., ... Bruchas, Michael R. (2015). Distinct Subpopulations of Nucleus Accumbens Dynorphin Neurons Drive Aversion and Reward. Neuron, 87(5), 1063-1077. doi: http://dx.doi.org/10.1016/j.neuron.2015.08.019

Andlin-Sobocki, P., \& Rehm, J. (2005). Cost of addiction in Europe. European Journal of Neurology, 12, 28-33. doi: 10.1111/j.1468-1331.2005.01194.x

Andrews, P. W., Damjanov, I., Simon, D., Banting, G. S., Carlin, C., Dracopoli, N. C., \& Føgh, J. (1984). Pluripotent embryonal carcinoma clones derived from the human teratocarcinoma cell line Tera-2. Differentiation in vivo and in vitro. Laboratory investigation; a journal of technical methods and pathology, 50(2), 147-162.

Arjune, D., \& Bodnar, R. J. (1990). Suppression of nocturnal, palatable and glucoprivic intake in rats by the $\mathrm{k}$ opioid antagonist, nor-binaltorphamine. Brain Res, 534(1-2), 313-316. doi: http://dx.doi.org/10.1016/0006-8993(90)90147-4

Arnt, J., Bøgeso, K. P., Hyttel, J., \& Meier, E. (1988). Relative Dopamine D1 and D2 Receptor Affinity and Efficacy Determine Whether Dopamine Agonists Induce Hyperactivity or Oral Stereotypy in Rats. Pharmacology \& Toxicology, 62(3), 121-130. doi: 10.1111/j.1600-0773.1988.tb01859.x

Balster, R. L. (1991). Drug abuse potential evaluation in animals. British Journal of Addiction, 86(12), 1549-1558.

Bardo, M. T., \& Bevins, R. A. (2000). Conditioned place preference: what does it add to our preclinical understanding of drug reward? Psychopharmacology, 153(1), 31-43. doi: $10.1007 / \mathrm{s} 002130000569$

Beardsley, P., Howard, J., Shelton, K., \& Carroll, F. I. (2005). Differential effects of the novel kappa opioid receptor antagonist, JDTic, on reinstatement of cocaine-seeking induced by footshock stressors vs cocaine primes and its antidepressant-like effects in rats.

Psychopharmacology, 183(1), 118-126. doi: 10.1007/s00213-005-0167-4

Béguin, C., Duncan, K. K., Munro, T. A., Ho, D. M., Xu, W., Liu-Chen, L.-Y., . . Cohen, B. M. (2009). Modification of the furan ring of salvinorin A: Identification of a selective partial agonist at the kappa opioid receptor. Bioorganic \& Medicinal Chemistry, 17(3), 1370-1380. doi: http://dx.doi.org/10.1016/i.bmc.2008.12.012

Bera, I., \& Ghoshal, N. (2014). Positively Charged Nitrogen is Not Indispensable Requirement for Binding of Nitrogenous kappa-Opioid Agonists: Insights from Docking Studies. Letters in Drug Design \& Discovery, 11(6), 809-813. 
BERL-Economics. (2009). Costs of harmful alcohol and other drug use. Report to the Ministry of Health and ACC. Wellington: BERL Economics.

Bolan, E. A., Kivell, B., Jaligam, V., Oz, M., Jayanthi, L. D., Han, Y., ... Shippenberg, T. S. (2007). D2 Receptors Regulate Dopamine Transporter Function via an Extracellular SignalRegulated Kinases 1 and 2-Dependent and Phosphoinositide 3 Kinase-Independent Mechanism. Mol Pharmacol, 71(5), 1222-1232. doi: 10.1124/mol.106.027763

Bolan, E. A., Kivell, B., Jaligam, V., Oz, M., Jayanthi, L. D., Han, Y., .. Shippenberg, T. S. (2007). D2 receptors regulate dopamine transporter function via an extracellular signal-regulated kinases 1 and 2-dependent and phosphoinositide 3 kinase-independent mechanism. Mol Pharmacol, 71(5), 1222-1232. doi: DOI 10.1124/mol.106.027763

Borsini, F., \& Meli, A. (1988). Is the forced swimming test a suitable model for revealing antidepressant activity? Psychopharmacology, 94(2), 147-160. doi: 10.1007/BF00176837

Bosch, P. (2013). The preclinical study of methamphetamine self-administartion and the underlying molecular biology and proteomics changes in the reward system. (Doctor of Philosophy), Victoria University of Wellington, Wellington.

Bossert, J., Marchant, N., Calu, D., \& Shaham, Y. (2013). The reinstatement model of drug relapse: recent neurobiological findings, emerging research topics, and translational research. Psychopharmacology, 229(3), 453-476. doi: 10.1007/s00213-013-3120-y

Braida, D., Capurro, V., Zani, A., Rubino, T., Viganò, D., Parolaro, D., \& Sala, M. (2009). Potential anxiolytic- and antidepressant-like effects of salvinorin $A$, the main active ingredient of Salvia divinorum, in rodents. British Journal of Pharmacology, 157(5), 844-853. doi: 10.1111/j.1476-5381.2009.00230.x

Braida, D., Limonta, V., Pegorini, S., Zani, A., Guerini-Rocco, C., Gori, E., \& Sala, M. (2007). Hallucinatory and rewarding effect of salvinorin A in zebrafish: K-opioid and CB1cannabinoid receptor involvement. Psychopharmacology, 190(4), 441-448. doi: 10.1007/s00213-006-0639-1

Bruchas, M. R., \& Chavkin, C. (2010). Kinase cascades and ligand-directed signaling at the kappa opioid receptor. Psychopharmacology, 210(2), 137-147. doi: 10.1007/s00213-010-1806-y

Bruchas, M. R., Land, B. B., \& Chavkin, C. (2010). The dynorphin/kappa opioid system as a modulator of stress-induced and pro-addictive behaviors. Brain Res, 1314, 44-55. doi: 10.1016/j.brainres.2009.08.062

Bruchas, M. R., Schindler, A. G., Shankar, H., Messinger, D. I., Miyatake, M., Land, B. B., . . Chavkin, C. (2011). Selective p38alpha MAPK deletion in serotonergic neurons produces stress resilience in models of depression and addiction. Neuron, 71(3), 498-511. doi: 10.1016/j.neuron.2011.06.011

Butelman, E. R., Caspers, M., Lovell, K. M., Kreek, M. J., \& Prisinzano, T. E. (2012). Behavioral effects and central nervous system levels of the broadly available kappa-agonist hallucinogen salvinorin A are affected by P-glycoprotein modulation in vivo. J Pharmacol Exp Ther, 341(3), 802-808. doi: 10.1124/jpet.112.193227

Butelman, E. R., Mandau, M., Tidgewell, K., Prisinzano, T. E., Yuferov, V., \& Kreek, M. J. (2007). Effects of salvinorin $A$, a kappa-opioid hallucinogen, on a neuroendocrine biomarker assay in nonhuman primates with high kappa-receptor homology to humans. $J$ Pharmacol Exp Ther, 320(1), 300-306. doi: 10.1124/jpet.106.112417

Butelman, E. R., Prisinzano, T. E., Deng, H., Rus, S., \& Kreek, M. J. (2009). Unconditioned behavioral effects of the powerful kappa-opioid hallucinogen salvinorin $A$ in nonhuman primates: fast onset and entry into cerebrospinal fluid. J Pharmacol Exp Ther, 328(2), 588-597. doi: 10.1124/jpet.108.145342

Butelman, E. R., Rus, S., Prisinzano, T. E., \& Kreek, M. J. (2010). The discriminative effects of the kappa-opioid hallucinogen salvinorin A in nonhuman primates: dissociation from classic hallucinogen effects. Psychopharmacology (Berl), 210(2), 253-262. doi: 10.1007/s00213009-1771-5 
Cachat, J., Kyzar, E. J., Collins, C., Gaikwad, S., Green, J., Roth, A., . . Kalueff, A. V. (2013). Unique and potent effects of acute ibogaine on zebrafish: The developing utility of novel aquatic models for hallucinogenic drug research. Behavioural Brain Research, 236, 258-269. doi: http://dx.doi.org/10.1016/j.bbr.2012.08.041

Campbell, U. C., \& Carroll, M. E. (2000). Acquisition of drug self-administration: environmental and pharmacological interventions. Exp Clin Psychopharmacol, 8(3), 312-325.

Cappendijk, S. L. T., \& Dzoljic, M. R. (1993). Inhibitory effects of ibogaine on cocaine selfadministration in rats. Eur J Pharmacol, 241(2-3), 261-265. doi: http://dx.doi.org/10.1016/0014-2999(93)90212-Z

Carlezon, W. A., Jr., Béguin, C., DiNieri, J. A., Baumann, M. H., Richards, M. R., Todtenkopf, M. S., ... Cohen, B. M. (2006). Depressive-Like Effects of the k-Opioid Receptor Agonist Salvinorin A on Behavior and Neurochemistry in Rats. Journal of Pharmacology and Experimental Therapeutics, 316(1), 440-447. doi: 10.1124/jpet.105.092304

Carlezon, W. A., Jr., Thome, J., Olson, V. G., Lane-Ladd, S. B., Brodkin, E. S., Hiroi, N., . . Nestler, E. J. (1998). Regulation of cocaine reward by CREB. Science, 282(5397), 2272-2275.

Carlsson, A., Lindqvist, M., \& Magnusson, T. O. R. (1957). 3,4-Dihydroxyphenylalanine and 5Hydroxytryptophan as Reserpine Antagonists. Nature, 180(4596), 1200-1200.

Cartwright, W. S. (2008). Economic costs of drug abuse: Financial, cost of illness, and services. Journal of Substance Abuse Treatment, 34(2), 224-233. doi: 10.1016/j.jsat.2007.04.003

Cass, W. A., Gerhardt, G. A., Mayfield, R. D., Curella, P., \& Zahniser, N. R. (1992). Differences in dopamine clearance and diffusion in rat striatum and nucleus accumbens following systemic cocaine administration. Journal of Neurochemistry, 59(1), 259-266. doi: http://onlinelibrary.wiley.com/doi/10.1111/j.1471-4159.1992.tb08899.x/abstract

Castro, D. C., \& Berridge, K. C. (2014). Opioid Hedonic Hotspot in Nucleus Accumbens Shell: Mu, Delta, and Kappa Maps for Enhancement of Sweetness "Liking" and "Wanting". The Journal of Neuroscience, 34(12), 4239-4250. doi: 10.1523/jneurosci.4458-13.2014

Chartoff, E. H., Ebner, S. R., Sparrow, A., Potter, D., Baker, P. M., Ragozzino, M. E., \& Roitman, M. F. (2015). Relative Timing Between Kappa Opioid Receptor Activation and Cocaine Determines the Impact on Reward and Dopamine Release. Neuropsychopharmacology. doi: $10.1038 / n p p .2015 .226$

Chartoff, E. H., Potter, D., Damez-Werno, D., Cohen, B. M., \& Carlezon, W. A., Jr. (2008). Exposure to the selective kappa-opioid receptor agonist salvinorin $A$ modulates the behavioral and molecular effects of cocaine in rats. Neuropsychopharmacology, 33(11), 2676-2687. doi: 10.1038/sj.npp.1301659

Chartoff, E. H., Sawyer, A., Rachlin, A., Potter, D., Pliakas, A., \& Carlezon, W. A. (2012). Blockade of kappa opioid receptors attenuates the development of depressive-like behaviors induced by cocaine withdrawal in rats. Neuropharmacology, 62(1), 167-176. doi: 10.1016/j.neuropharm.2011.06.014

Chen, B. T., Bowers, M. S., Martin, M., Hopf, F. W., Guillory, A. M., Carelli, R. M., . . Bonci, A. (2008). Cocaine but Not Natural Reward Self-Administration nor Passive Cocaine Infusion Produces Persistent LTP in the VTA. Neuron, 59(2), 288-297. doi:

http://dx.doi.org/10.1016/j.neuron.2008.05.024

Childress, A. R., Hole, A. V., Ehrman, R. N., Robbins, S. J., McLellan, A. T., \& O'Brien, C. P. (1993). Cue reactivity and cue reactivity interventions in drug dependence. NIDA research monograph, 137, 73-95.

Ciliax, B. J., Drash, G. W., Staley, J. K., Haber, S., Mobley, C. J., Miller, G. W., ... Levey, A. I. (1999). Immunocytochemical localization of the dopamine transporter in human brain. The Journal of Comparative Neurology, 409(1), 38-56. doi: 10.1002/(SICI)10969861(19990621)409:1<38::AID-CNE4>3.0.CO;2-1 
Ciliax, B. J., Heilman, C., Demchyshyn, L., Pristupa, Z., Ince, E., Hersch, S., . . Levey, A. (1995). The dopamine transporter: immunochemical characterization and localization in brain. The Journal of Neuroscience, 15(3), 1714-1723.

Collins, S. L., Kunko, P. M., Ladenheim, B., Cadet, J. L., Carroll, F. I., \& Izenwasser, S. (2002). Chronic cocaine increases kappa-opioid receptor density: Lack of effect by selective dopamine uptake inhibitors. Synapse, 45(3), 153-158. doi: 10.1002/syn.10091

Cortez, A. M., Charntikov, S., Der-Ghazarian, T., Horn, L. R., Crawford, C. A., \& McDougall, S. A. (2010). Age-dependent effects of $\mathrm{K}$-opioid receptor stimulation on cocaine-induced stereotyped behaviors and dopamine overflow in the caudate-putamen: an in vivo microdialysis study. Neuroscience, 169(1), 203-213. doi: http://dx.doi.org/10.1016/i.neuroscience.2010.04.052

Crawford, C. A., McDougall, S. A., Bolanos, C. A., Hall, S., \& Berger, S. P. (1995). The effects of the kappa agonist $U-50,488$ on cocaine-induced conditioned and unconditioned behaviors and Fos immunoreactivity. Psychopharmacology, 120(4), 392-399. doi: 10.1007/BF02245810

Cryan, J. F., Mombereau, C., \& Vassout, A. (2005). The tail suspension test as a model for assessing antidepressant activity: Review of pharmacological and genetic studies in mice. Neuroscience \& Biobehavioral Reviews, 29(4-5), 571-625. doi: http://dx.doi.org/10.1016/i.neubiorev.2005.03.009

Dackis, C. A., \& Gold, M. S. (1985). New concepts in cocaine addiction: The dopamine depletion hypothesis. Neuroscience \& Biobehavioral Reviews, 9(3), 469-477. doi: http://dx.doi.org/10.1016/0149-7634(85)90022-3

Dasgupta, A., Chow, L., Tso, G., \& Nazareno, L. (2003). Stability of NT-proBNP in serum specimens collected in Becton Dickinson Vacutainer (SST) tubes. Clin Chem, 49(6 Pt 1), 958-960.

Davis, W. M., \& Smith, S. (1976). Role of conditioned reinforcers in the initiation, maintenance and extinction of drug-seeking behavior. The Pavlovian journal of biological science : official journal of the Pavlovian, 11(4), 222-236. doi: 10.1007/BF03000316

Daws, L. C., Callaghan, P. D., Morón, J. A., Kahlig, K. M., Shippenberg, T. S., Javitch, J. A., \& Galli, A. (2002). Cocaine Increases Dopamine Uptake and Cell Surface Expression of Dopamine Transporters. Biochemical and Biophysical Research Communications, 290(5), 15451550. doi: http://dx.doi.org/10.1006/bbrc.2002.6384

de Wit, H. (1996). Priming effects with drugs and other reinforcers. Experimental and Clinical Psychopharmacology, 4(1), 5-10. doi: 10.1037/1064-1297.4.1.5

de Wit, H., \& Stewart, J. (1981). Reinstatement of cocaine-reinforced responding in the rat. Psychopharmacology, 75(2), 134-143.

Delfs, J., Schreiber, L., \& Kelley, A. (1990). Microinjection of cocaine into the nucleus accumbens elicits locomotor activation in the rat. The Journal of Neuroscience, 10(1), 303-310.

Depoortere, R. Y., Li, D. H., Lane, J. D., \& Emmett-Oglesby, M. W. (1993). Parameters of selfadministration of cocaine in rats under a progressive-ratio schedule. Pharmacology Biochemistry and Behavior, 45(3), 539-548. doi: http://dx.doi.org/10.1016/00913057(93)90503-L

DeWire, S. M., Yamashita, D. S., Rominger, D. H., Liu, G., Cowan, C. L., Graczyk, T. M., . . Violin, J. D. (2013). A G protein-biased ligand at the mu-opioid receptor is potently analgesic with reduced gastrointestinal and respiratory dysfunction compared with morphine. $J$ Pharmacol Exp Ther, 344(3), 708-717. doi: 10.1124/jpet.112.201616

Di Chiara, G., \& Imperato, A. (1988). Drugs abused by humans preferentially increase synaptic dopamine concentrations in the mesolimbic system of freely moving rats. Proceedings of the National Academy of Sciences, 85(14), 5274-5278. 
Dunham, N. W., \& Miya, T. S. (1957). A note on a simple apparatus for detecting neurological deficit in rats and mice. Journal of the American Pharmaceutical Association, 46(3), 208209. doi: 10.1002/jps.3030460322

Durieux, P. F., Bearzatto, B., Guiducci, S., Buch, T., Waisman, A., Zoli, M., ... De Kerchove D'exaerde, A. (2009). D2R striatopallidal neurons inhibit both locomotor and drug reward processes. Nature Neuroscience, 12(4), 393-395. doi: http://dx.doi.org/10.1038/nn.2286

Ebner, S. R., Roitman, M. F., Potter, D. N., Rachlin, A. B., \& Chartoff, E. H. (2010). Depressive-like effects of the kappa opioid receptor agonist salvinorin $A$ are associated with decreased phasic dopamine release in the nucleus accumbens. Psychopharmacology, 210(2), 241252. doi: $10.1007 / \mathrm{s} 00213-010-1836-5$

Epstein, D. H., Preston, K. L., Stewart, J., \& Shaham, Y. (2006). Toward a model of drug relapse: an assessment of the validity of the reinstatement procedure. Psychopharmacology, 189(1), 1-16. doi: DOI 10.1007/s00213-006-0529-6

Eriksson, P. S., Nilsson, M., Wagberg, M., Hansson, E., \& Ronnback, L. (1993). Kappa-opioid receptors on astrocytes stimulate L-type $\mathrm{Ca}^{2+}$ channels. Neuroscience, 54(2), 401-407. doi: 10.1016/0306-4522(93)90261-d

Fink, H., Morgenstern, R., \& Oelssner, W. (1979). Psychotomimetics potentiate locomotor hyperactivity induced by dopaminergic drugs. Pharmacology Biochemistry and Behavior, 11(5), 479-482. doi: http://dx.doi.org/10.1016/0091-3057(79)90028-5

Fleisher, L., \& Glick, S. (1979). Hallucinogen-induced rotational behavior in rats. Psychopharmacology, 62(2), 193-200. doi: 10.1007/BF00427136

Flynn, P. M., Kristiansen, P. L., Porto, J. V., \& Hubbard, R. L. (1999). Costs and benefits of treatment for cocaine addiction in DATOS. Drug Alcohol Depend, 57(2), 167-174. doi: http://dx.doi.org/10.1016/S0376-8716(99)00083-6

Freeman, K. B., Naylor, J. E., Prisinzano, T. E., \& Woolverton, W. L. (2014). Assessment of the kappa opioid agonist, salvinorin $A$, as a punisher of drug self-administration in monkeys. Psychopharmacology (Berl). doi: 10.1007/s00213-014-3436-2

Funk, D., Coen, K., \& Lê, A. D. (2014). The role of kappa opioid receptors in stress-induced reinstatement of alcohol seeking in rats. Brain and Behavior, 4(3), 356-367. doi: 10.1002/brb3.222

Gehrke, B. J., Chefer, V. I., \& Shippenberg, T. S. (2008). Effects of acute and repeated administration of salvinorin $A$ on dopamine function in the rat dorsal striatum. Psychopharmacology, 197(3), 509-517. doi: 10.1007/s00213-007-1067-6

Geyer, M., Light, R., Rose, G., Petersen, L., Horwitt, D., Adams, L., \& Hawkins, R. (1979). A characteristic effect of hallucinogens on investigatory responding in rats. Psychopharmacology, 65(1), 35-40. doi: 10.1007/BF00491975

Glantz, M. D., Anthony, J. C., Berglund, P. A., Degenhardt, L., Dierker, L., Kalaydjian, A., . . Kessler, R. C. (2009). Mental disorders as risk factors for later substance dependence: estimates of optimal prevention and treatment benefits. Psychological Medicine, 39(8), 1365-1377. doi: 10.1017/s0033291708004510

Glick, S. D., Maisonneuve, I. M., Raucci, J., \& Archer, S. (1995). Kappa opioid inhibition of morphine and cocaine self-administration in rats. Brain Res, 681, 147-152.

Graham, D., Hoppenot, R., Hendryx, A., \& Self, D. (2007). Differential ability of D1 and D2 dopamine receptor agonists to induce and modulate expression and reinstatement of cocaine place preference in rats. Psychopharmacology, 191(3), 719-730. doi: 10.1007/s00213-006-0473-5

Graham, F. L., Smiley, J., Russell, W. C., \& Nairn, R. (1977). Characteristics of a Human Cell Line Transformed by DNA from Human Adenovirus Type 5. Journal of General Virology, 36(1), 59-72. doi: doi:10.1099/0022-1317-36-1-59 
Grant, B. F. (1996). Prevalence and correlates of drug use and DSM-IV drug dependence in the United States: results of the National Longitudinal Alcohol Epidemiologic Survey. Journal of Substance Abuse, 8(2), 195-210. doi: http://dx.doi.org/10.1016/S08993289(96)90249-7

Griffiths, R., Findley, J., Brady, J., Dolan-Gutcher, K., \& Robinson, W. (1975). Comparison of progressive-ratio performance maintained by cocaine, methylphenidate and secobarbital. Psychopharmacologia, 43(1), 81-83. doi: 10.1007/BF00437619

Groer, C. E., Tidgewell, K., Moyer, R. A., Harding, W. W., Rothman, R. B., Prisinzano, T. E., \& Bohn, L. M. (2007). An Opioid Agonist that Does Not Induce $\mu$-Opioid Receptor-Arrestin Interactions or Receptor Internalization. Mol Pharmacol, 71(2), 549-557. doi: 10.1124/mol.106.028258

Grossman, L., Utterback, E., Stewart, A., Gaikwad, S., Chung, K. M., Suciu, C., . . Kalueff, A. V. (2010). Characterization of behavioral and endocrine effects of LSD on zebrafish. Behavioural Brain Research, 214(2), 277-284. doi: http://dx.doi.org/10.1016/j.bbr.2010.05.039

Grudt, T. J., \& Williams, J. T. (1993). Kappa-opioid receptors also increase potassium conductance. Proceedings of the National Academy of Sciences of the United States of America, 90(23), 11429-11432. doi: 10.1073/pnas.90.23.11429

Hall, W., Teesson, M., Lynskey, M., \& Degenhardt, L. (1999). The 12-month prevalence of substance use and ICD-10 substance use disorders in Australian adults: findings from the National Survey of Mental Health and Well-Being. Addiction, 94(10), 1541-1550. doi: 10.1046/j.1360-0443.1999.9410154110.x

Harding, W. W., Schmidt, M., Tidgewell, K., Kannan, P., Holden, K. G., Dersch, C. M., . . Prisinzano, T. E. (2006). Synthetic studies of neoclerodane diterpenes from Salvia divinorum: Selective modification of the furan ring. Bioorg Med Chem Lett, 16(12), 31703174. doi: http://dx.doi.org/10.1016/j.bmcl.2006.03.062

Harding, W. W., Schmidt, M., Tidgewell, K., Kannan, P., Holden, K. G., Gilmour, B., . . Prisinzano, T. E. (2006). Synthetic studies of neoclerodane diterpenes from Salvia divinorum: semisynthesis of salvinicins $A$ and $B$ and other chemical transformations of salvinorin $A$. Journal of Natural Products, 69(1), 107-112. doi: 10.1021/np050398i

Harding, W. W., Tidgewell, K., Byrd, N., Cobb, H., Dersch, C. M., Butelman, E. R., ... Prisinzano, T. E. (2005). Neoclerodane Diterpenes as a Novel Scaffold for $\mu$ Opioid Receptor Ligandst. Journal of Medicinal Chemistry, 48(15), 4765-4771. doi: 10.1021/jm048963m

Harwood, H. J., Fountain, D., \& Livermore, G. (1999). Economic cost of alcohol and drug abuse in the United States, 1992: a report. Addiction, 94(5), 631-635.

Hawkins, J., Hicks, R. A., Phillips, N., \& Moore, J. D. (1978). Swimming rats and human depression. Nature, 274(5670), 512-512.

Heidbreder, C. A., Babovic-Vuksanovic, D., Shoaib, M., \& Shippenberg, T. S. (1995). Development of behavioral sensitization to cocaine: influence of kappa opioid receptor agonists. Journal of Pharmacology and Experimental Therapeutics, 275(1), 150-163.

Heidbreder, C. A., Goldberg, S. R., \& Shippenberg, T. S. (1993). The kappa-opioid receptor agonist U-69593 attenuates cocaine-induced behavioral sensitization in the rat. Brain Res, 616(1-2), 335-338. doi: 10.1016/0006-8993(93)90228-f

Heidbreder, C. A., \& Shippenberg, T. S. (1994). U-69593 prevents cocaine sensitization by normalizing basal accumbens dopamine. Neuroreport, 5(14), 1797-1800.

Herrmann, Z., Uhl, W., Steinberg, H. W., \& Dworschack, R. (2003). The influence of renal function on NT-proBNP levels in various disease groups. Clin Lab, 49(11-12), 649-656.

Hodos, W. (1961). Progressive Ratio as a Measure of Reward Strength. Science, 134(3483), 943944. doi: 10.1126/science.134.3483.943

Hollander, J., Pham, D., Fowler, C., \& Kenny, P. J. (2012). Hypocretin-1 receptors regulate the reinforcing and reward-enhancing effects of cocaine: Pharmacological and behavioral 
genetics evidence. Frontiers in Behavioral Neuroscience, 6. doi: 10.3389/fnbeh.2012.00047

Hooker, J. M., Munro, T. A., Béguin, C., Alexoff, D., Shea, C., Xu, Y., \& Cohen, B. M. (2009). Salvinorin $A$ and derivatives: Protection from metabolism does not prolong short-term, whole-brain residence. Neuropharmacology, 57(4), 386-391. doi: http://dx.doi.org/10.1016/j.neuropharm.2009.06.044

Hooker, J. M., Xu, Y., Schiffer, W., Shea, C., Carter, P., \& Fowler, J. S. (2008). Pharmacokinetics of the potent hallucinogen, salvinorin $A$ in primates parallels the rapid onset and short duration of effects in humans. Neurolmage, 41(3), 1044-1050. doi: http://dx.doi.org/10.1016/j.neuroimage.2008.03.003

Hunter, J. C., Leighton, G. E., Meecham, K. G., Boyle, S. J., Horwell, D. C., Rees, D. C., \& Hughes, J. (1990). Cl-977, a novel and selective agonist for the k-opioid receptor. British Journal of Pharmacology, 101(1), 183-189. doi: 10.1111/j.1476-5381.1990.tb12110.x

Hurd, Y. L., \& Herkenham, M. (1993). Molecular alterations in the neostriatum of human cocaine addicts. Synapse, 13(4), 357-369. doi: 10.1002/syn.890130408

Ito, R., \& Hayen, A. (2011). Opposing Roles of Nucleus Accumbens Core and Shell Dopamine in the Modulation of Limbic Information Processing. The Journal of Neuroscience, 31(16), 6001-6007. doi: 10.1523/jneurosci.6588-10.2011

Jones, G. H., Marsden, C. A., \& Robbins, T. W. (1990). Increased sensitivity to amphetamine and reward-related stimuli following social isolation in rats: possible disruption of dopaminedependent mechanisms of the nucleus accumbens. Psychopharmacology, 102(3), 364372. doi: $10.1007 /$ BF02244105

Jordan, B. A., \& Devi, L. A. (1999). G-protein-coupled receptor heterodimerization modulates receptor function. Nature, 399(6737), 697-700.

Kam, A. Y. F., Chan, A. S. L., \& Wong, Y. H. (2004). k-Opioid Receptor Signals through Src and Focal Adhesion Kinase to Stimulate c-Jun N-Terminal Kinases in Transfected COS-7 Cells and Human Monocytic THP-1 Cells. Journal of Pharmacology and Experimental Therapeutics, 310(1), 301-310. doi: 10.1124/jpet.104.065078

Kane, B. E., McCurdy, C. R., \& Ferguson, D. M. (2008). Toward a Structure-Based Model of Salvinorin A Recognition of the k-Opioid Receptor. Journal of Medicinal Chemistry, 51(6), 1824-1830. doi: 10.1021/jm701040v

Kebabian, J. W. (1978). Multiple classes of dopamine receptors in mammalian central nervous system: the involvement of dopamine-sensitive adenylyl cyclase. Life Sciences, 23(5), 479-483. doi: 10.1016/0024-3205(78)90157-1

Kenakin, T. (2011). Functional Selectivity and Biased Receptor Signaling. J Pharmacol Exp Ther, 336(2), 296-302. doi: 10.1124/jpet.110.173948

Killinger, B. A., Peet, M. M., \& Baker, L. E. (2010). Salvinorin A fails to substitute for the discriminative stimulus effects of LSD or ketamine in Sprague-Dawley rats.

Pharmacology Biochemistry and Behavior, 96(3), 260-265. doi: http://dx.doi.org/10.1016/j.pbb.2010.05.014

Kim, T.-i., McCall, J. G., Jung, Y. H., Huang, X., Siuda, E. R., Li, Y., . . Bruchas, M. R. (2013). Injectable, Cellular-Scale Optoelectronics with Applications for Wireless Optogenetics. Science, 340(6129), 211-216. doi: 10.1126/science.1232437

Kivell, B., Uzelac, Z., Sundaramurthy, S., Rajamanickam, J., Ewald, A., Chefer, V., . . Shippenberg, T. S. (2014). Salvinorin A regulates dopamine transporter function via a kappa opioid receptor and ERK1/2-dependent mechanism. Neuropharmacology, 86(0), 228-240. doi: http://dx.doi.org/10.1016/j.neuropharm.2014.07.016

Klebe, R. J., \& Ruddle, R. H. (1969). Neuroblastoma - cell culture analysis of a differentiating stem cell system Journal of Cell Biology, 43(2P2), A69-\&.

Koob, G. F., \& Bloom, F. (1988). Cellular and molecular mechanisms of drug dependence. Science, 242(4879), 715-723. doi: 10.1126/science.2903550 
Koob, G. F., \& Moal, M. L. (1997). Drug Abuse: Hedonic Homeostatic Dysregulation. Science, 278(5335), 52-58. doi: $10.2307 / 2894498$

Kyzar, E. J., Collins, C., Gaikwad, S., Green, J., Roth, A., Monnig, L., . . Kalueff, A. V. (2012). Effects of hallucinogenic agents mescaline and phencyclidine on zebrafish behavior and physiology. Progress in Neuro-Psychopharmacology and Biological Psychiatry, 37(1), 194-202. doi: http://dx.doi.org/10.1016/j.pnpbp.2012.01.003

Land, B. B., Bruchas, M. R., Lemos, J. C., Xu, M., Melief, E. J., \& Chavkin, C. (2008). The dysphoric component of stress is encoded by activation of the dynorphin kappa-opioid system. J Neurosci, 28(2), 407-414. doi: 10.1523/JNEUROSCI.4458-07.2008

Land, B. B., Bruchas, M. R., Schattauer, S., Giardino, W. J., Aita, M., Messinger, D., . . Chavkin, C. (2009). Activation of the Kappa Opioid Receptor in the Dorsal Raphe Nucleus Mediates the Aversive Effects of Stress and Reinstates Drug Seeking. Proceedings of the National Academy of Sciences of the United States of America, 106(45), 19168-19173. doi: $10.2307 / 25593161$

Lange, J. E., Reed, M. B., Croff, J. M. K., \& Clapp, J. D. (2008). College student use of Salvia divinorum. Drug Alcohol Depend, 94(1-3), 263-266. doi: 10.1016/j.drugalcdep.2007.10.018

Le Naour, M., Lunzer, M. M., Powers, M. D., Kalyuzhny, A. E., Benneyworth, M. A., Thomas, M. J., \& Portoghese, P. S. (2014). Putative Kappa Opioid Heteromers As Targets for Developing Analgesics Free of Adverse Effects. Journal of Medicinal Chemistry, 57(15), 6383-6392.

Lenoir, M., Serre, F., Cantin, L., \& Ahmed, S. H. (2007). Intense Sweetness Surpasses Cocaine Reward. PLoS One, 2(8), e698. doi: 10.1371/journal.pone.0000698

Li, J.-G., Luo, L.-Y., Krupnick, J. G., Benovic, J. L., \& Liu-Chen, L.-Y. (1999). U50,488H-induced Internalization of the Human к Opioid Receptor Involves a $\beta$-Arrestin- and Dynamindependent Mechanism: $\mathrm{K}$ RECEPTOR INTERNALIZATION IS NOT REQUIRED FOR MITOGEN-ACTIVATED PROTEIN KINASE ACTIVATION. Journal of Biological Chemistry, 274(17), 12087-12094. doi: 10.1074/jbc.274.17.12087

Li, Y., \& van den Pol, A. N. (2006). Differential Target-Dependent Actions of Coexpressed Inhibitory Dynorphin and Excitatory Hypocretin/Orexin Neuropeptides. The Journal of Neuroscience, 26(50), 13037-13047. doi: 10.1523/jneurosci.3380-06.2006

Little, K. Y., Krolewski, D. M., Zhang, L., \& Cassin, B. J. (2003). Loss of striatal vesicular monoamine transporter protein (VMAT2) in human cocaine users. The American Journal of Psychiatry, 160(1), 47-55.

Magrassi, L., Purves, D., \& Lichtman, J. (1987). Fluorescent probes that stain living nerve terminals. The Journal of Neuroscience, 7(4), 1207-1214.

Mansour, A., Khachaturian, H., Lewis, M., Akil, H., \& Watson, S. (1987). Autoradiographic differentiation of $\mathrm{mu}$, delta, and kappa opioid receptors in the rat forebrain and midbrain. The Journal of Neuroscience, 7(8), 2445-2464.

Margolis, E. B., Lock, H., Chefer, V. I., Shippenberg, T. S., Hjelmstad, G. O., \& Fields, H. L. (2006). kappa opioids selectively control dopaminergic neurons projecting to the prefrontal cortex. Proceedings of the National Academy of Sciences of the United States of America, 103(8), 2938-2942. doi: 10.1073/pnas.0511159103

Mason, K., Hewitt, A., Stefanogiannis, N., Bhattacharya, A., Yeh, L. C., \& Devlin, M. (2010). Drug use in New Zealand: Key Results of the 2007/08 New Zealand Alcohol and Drug Use Survey. Wellington, New Zealand: Ministry of Health.

McCurdy, C. R., Sufka, K. J., Smith, G. H., Warnick, J. E., \& Nieto, M. J. (2006). Antinociceptive profile of salvinorin $A$, a structurally unique kappa opioid receptor agonist.

Pharmacology Biochemistry and Behavior, 83(1), 109-113. doi: http://dx.doi.org/10.1016/i.pbb.2005.12.011

McGregor, A., Lacosta, S., \& Roberts, D. C. S. (1993). L-Tryptophan decreases the breaking point under a progressive ratio schedule of intravenous cocaine reinforcement in the rat. 
Pharmacology Biochemistry and Behavior, 44(3), 651-655. doi:

http://dx.doi.org/10.1016/0091-3057(93)90181-R

McLennan, G. P., Kiss, A., Miyatake, M., Belcheva, M. M., Chambers, K. T., Pozek, J. J., . . Coscia, C. J. (2008). Kappa opioids promote the proliferation of astrocytes via $\mathrm{G} \beta \gamma$ and $\beta$-arrestin 2-dependent MAPK-mediated pathways. Journal of Neurochemistry, 107(6), 1753-1765. doi: 10.1111/j.1471-4159.2008.05745.x

Mello, N. K., \& Negus, S. S. (2000). Interactions between Kappa Opioid Agonists and Cocaine: Preclinical Studies. Annals of the New York Academy of Sciences, 909(1), 104-132. doi: 10.1111/j.1749-6632.2000.tb06678.x

Mendrek, A., Blaha, C. D., \& Phillips, A. G. (1998). Pre-exposure of rats to amphetamine sensitizes self-administration of this drug under a progressive ratio schedule. Psychopharmacology, 135(4), 416-422. doi: 10.1007/s002130050530

Morani, A. S., Ewald, A., Prevatt-Smith, K. M., Prisinzano, T. E., \& Kivell, B. M. (2013). The 2methoxy methyl analogue of salvinorin $A$ attenuates cocaine-induced drug seeking and sucrose reinforcements in rats. Eur J Pharmacol, 720(1-3), 69-76. doi: DOI 10.1016/j.ejphar.2013.10.050

Morani, A. S., Kivell, B., Prisinzano, T. E., \& Schenk, S. (2009). Effect of kappa-opioid receptor agonists U69593, U50488H, spiradoline and salvinorin A on cocaine-induced drugseeking in rats. Pharmacology Biochemistry and Behavior, 94(2), 244-249. doi: DOI 10.1016/j.pbb.2009.09.002

Morani, A. S., Schenk, S., Prisinzano, T. E., \& Kivell, B. M. (2012). A single injection of a novel kappa opioid receptor agonist salvinorin $A$ attenuates the expression of cocaine-induced behavioral sensitization in rats. Behav Pharmacol, 23(2), 162-170. doi: 10.1097/FBP.0b013e3283512c1e

Morani, A. S., Schenk, S., Prisinzano, T. E., \& Kivell, B. M. (2012). A single injection of a novel kappa opioid receptor agonist salvinorin $A$ attenuates the expression of cocaine-induced behavioral sensitization in rats. Behav Pharmacol, 23(2), 162-170. doi: Doi 10.1097/Fbp.0b013e3283512c1e

Mueller, T., Gegenhuber, A., Poelz, W., \& Haltmayer, M. (2003). Comparison of the Biomedica NT-proBNP enzyme immunoassay and the Roche NT-proBNP chemiluminescence immunoassay: implications for the prediction of symptomatic and asymptomatic structural heart disease. Clin Chem, 49(6 Pt 1), 976-979.

Munro, T. A., Duncan, K. K., Xu, W., Wang, Y., Liu-Chen, L. Y., Carlezon, W. A., Jr., . . Beguin, C. (2008). Standard protecting groups create potent and selective kappa opioids: salvinorin B alkoxymethyl ethers. Bioorg Med Chem, 16(3), 1279-1286. doi: 10.1016/j.bmc.2007.10.067

Muschamp, J. W., Hollander, J. A., Thompson, J. L., Voren, G., Hassinger, L. C., Onvani, S., . . Carlezon, W. A. (2014). Hypocretin (orexin) facilitates reward by attenuating the antireward effects of its cotransmitter dynorphin in ventral tegmental area. Proceedings of the National Academy of Sciences, 111(16), E1648-E1655. doi: 10.1073/pnas.1315542111

Mysels, D., \& Sullivan, M. A. (2009). The kappa-opiate receptor impacts the pathophysiology and behavior of substance use. Am J Addict, 18(4), 272-276. doi: 10.1080/10550490902925862

Narendran, R., Lopresti, B. J., Martinez, D., Mason, N. S., Himes, M., May, M. A., . . Frankle, W. G. (2012). In Vivo Evidence for Low Striatal Vesicular Monoamine Transporter 2 (VMAT2) Availability in Cocaine Abusers. The American Journal of Psychiatry, 169(1), 55-63.

Navarro, G., Moreno, E., Bonaventura, J., Brugarolas, M., Farré, D., Aguinaga, D., . . McCormick, P. J. (2013). Cocaine Inhibits Dopamine D2 Receptor Signaling via Sigma-1-D2 Receptor Heteromers. PLoS One, 8(4), e61245. doi: 10.1371/journal.pone.0061245 
Naylor, J., Prisinzano, T., \& Freeman, K. (2015). Self-administration of oxycodone alone or as a mixture with the kappa agonist, salvinorin a, by monkeys under a progressive ratio schedule of reinforcement. Drug Alcohol Depend, 146(0), e48-e49. doi: http://dx.doi.org/10.1016/i.drugalcdep.2014.09.503

NDIC. (2011). National Drug Threat Assessment. Washington, D.C: U.S. Department of Justice. Negus, S. S. (2004). Effects of the kappa opioid agonist U50,488 and the kappa opioid antagonist nor-binaltorphimine on choice between cocaine and food in rhesus monkeys. Psychopharmacology, 176(2), 204-213. doi: http://dx.doi.org/10.1007/s00213-004-1878$\underline{7}$

Negus, S. S., Mello, N. K., Portoghese, P. S., \& Lin, C.-E. (1997). Effects of Kappa Opioids on Cocaine Self-Administration by Rhesus Monkeys. Journal of Pharmacology and Experimental Therapeutics, 282(1), 44-55.

O'Brien, C. P., Childress, A. R., McLellan, A. T., \& Ehrman, R. (1992). Classical Conditioning in Drug-Dependent Humansa. Annals of the New York Academy of Sciences, 654(1), 400415. doi: 10.1111/j.1749-6632.1992.tb25984.x

O'Neill, K. A., \& Valentino, D. (1982). Escapability and generalization: Effect on 'behavioral despair'. Eur J Pharmacol, 78(3), 379-380. doi: http://dx.doi.org/10.1016/00142999(82)90043-7

Ookuma, K., Barton, C., York, D. A., \& Bray, G. A. (1997). Effect of Enterostatin and KappaOpioids on Macronutrient Selection and Consumption. Peptides, 18(6), 785-791. doi: http://dx.doi.org/10.1016/S0196-9781(97)00029-6

Ortega, A., Blount, J. F., \& Manchand, P. S. (1982). Salvinorin, a new trans-neoclerodane diterpene from Salvia divinorum(Labiatae). Journal of the Chemical Society, Perkin Transactions 1(0), 2505-2508. doi: 10.1039/P19820002505

Ortiz de Montellano, P. R., \& Kunze, K. L. (1980). Self-catalyzed inactivation of hepatic cytochrome P-450 by ethynyl substrates. Journal of Biological Chemistry, 255(12), 55785585.

Oz, M., Libby, T., Kivell, B., Jaligam, V., Ramamoorthy, S., \& Shippenberg, T. S. (2010). Real-time, spatially resolved analysis of serotonin transporter activity and regulation using the fluorescent substrate, ASP(+). Journal of Neurochemistry, 114(4), 1019-1029. doi: DOI 10.1111/j.1471-4159.2010.06828.x

Panlilio, L. V., \& Goldberg, S. R. (2007). Self-administration of drugs in animals and humans as a model and an investigative tool. Addiction, 102(12), 1863-1870. doi: 10.1111/j.13600443.2007.02011.x

Park, K., Volkow, N. D., Pan, Y., \& Du, C. (2013). Chronic Cocaine Dampens Dopamine Signaling during Cocaine Intoxication and Unbalances D1 over D2 Receptor Signaling. The Journal of Neuroscience, 33(40), 15827-15836. doi: 10.1523/jneurosci.1935-13.2013

Peciña, S., Berridge, K. C., \& Parker, L. A. (1997). Pimozide Does Not Shift Palatability: Separation of Anhedonia from Sensorimotor Suppression by Taste Reactivity. Pharmacology Biochemistry and Behavior, 58(3), 801-811. doi: http://dx.doi.org/10.1016/S00913057(97)00044-0

Pfaus, J. G., Damsma, G., Nomikos, G. G., Wenkstern, D. G., Blaha, C. D., Phillips, A. G., \& Fibiger, H. C. (1990). Sexual behavior enhances central dopamine transmission in the male rat. Brain Res, 530(2), 345-348. doi: http://dx.doi.org/10.1016/0006-8993(90)91309-5

Pfeiffer, A., Brantl, V., Herz, A., \& Emrich, H. M. (1986). Psychotomimesis mediated by kappa opiate receptors. Science, 233(4765), 774-776. doi: 10.2307/1697305

Phillips, A. G., \& Fibiger, H. C. (1978). The role of dopamine in maintaining intracranial selfstimulation in the ventral tegmentum, nucleus accumbens, and medial prefrontal cortex. Canadian Journal of Psychology-Revue Canadienne De Psychologie, 32(2), 58-66. doi: 10.1037/h0081676 
Pickens, R., \& Thompson, T. (1968). COCAINE-REINFORCED BEHAVIOR IN RATS: EFFECTS OF REINFORCEMENT MAGNITUDE AND FIXED-RATIO SIZE. Journal of Pharmacology and Experimental Therapeutics, 161(1), 122-129.

Porsolt, R. D., Lepichon, M., \& Jalfre, M. (1977). Depression: a new animal model sensitive to antidepressant treatments. Nature, 266(5604), 730-732. doi: 10.1038/266730a0

Potter, D. N., Damez-Werno, D., Carlezon Jr, W. A., Cohen, B. M., \& Chartoff, E. H. (2011).

Repeated Exposure to the $\mathrm{k}$-Opioid Receptor Agonist Salvinorin A Modulates Extracellular Signal-Regulated Kinase and Reward Sensitivity. Biol Psychiatry, 70(8), 744753. doi: http://dx.doi.org/10.1016/j.biopsych.2011.05.021

Prendergast, M. L., Podus, D., Chang, E., \& Urada, D. (2002). The effectiveness of drug abuse treatment: a meta-analysis of comparison group studies. Drug Alcohol Depend, 67(1), 53-72. doi: http://dx.doi.org/10.1016/S0376-8716(02)00014-5

Prevatt-Smith, K. M., Lovell, K. M., Simpson, D. S., Day, V. W., Douglas, J. T., Bosch, P., . . Prisinzano, T. E. (2011). Potential drug abuse therapeutics derived from the hallucinogenic natural product salvinorin A. Medchemcomm, 2(12), 1217-1222. doi: Doi 10.1039/C1md00192b

Prisinzano, T. E., Tidgewell, K., \& Harding, W. W. (2005). k Opioids as potential treatments for stimulant dependence. The AAPS Journal, 7(3), E592-E599. doi: 10.1208/aapsj070361

Prisinzano, T. E., Tidgewell, K., \& Harding, W. W. (2008). k Opioids as Potential Treatments for Stimulant Dependence. In R. Rapaka \& W. Sadée (Eds.), Drug Addiction (pp. 231-245): Springer New York.

Pulvirenti, L., Berrier, R., Kreifeldt, M., \& Koob, G. K. (1994). Modulation of locomotor activity by NMDA receptors in the nucleus accumbens core and shell regions of the rat. Brain Res, 664(1), 231-236. doi: http://dx.doi.org/10.1016/0006-8993(94)91977-1

Quigley, B. M., \& Collins, R. L. (1999). The modeling of alcohol consumption: a meta-analytic review. Journal of Studies on Alcohol, 60(1), 90-98.

Redila, V. A., \& Chavkin, C. (2008). Stress-induced reinstatement of cocaine seeking is mediated by the kappa opioid system. Psychopharmacology, 200(1), 59-70. doi: 10.1007/s00213008-1122-y

Reece, P. A., Sedman, A. J., Rose, S., Wright, D. S., Dawkins, R., \& Rajagopalan, R. (1994). Diuretic Effects, Pharmacokinetics, and Safety of a New Centrally Acting Kappa-Opioid Agonist (Ci-977) in Humans. Journal of Clinical Pharmacology, 34(11), 1126-1132.

Reed, M. J., \& Fotherby, K. (1979). Intestinal absorption of synthetic steroids. Journal of Steroid Biochemistry, 11(2), 1107-1112. doi: http://dx.doi.org/10.1016/0022-4731(79)90160-2

Richardson, N. R., \& Roberts, D. C. S. (1996). Progressive ratio schedules in drug selfadministration studies in rats: a method to evaluate reinforcing efficacy. Journal of Neuroscience Methods, 66(1), 1-11. doi: http://dx.doi.org/10.1016/0165$\underline{0270(95) 00153-0}$

Riley, A. P., Day, V. W., Navarro, H. A., \& Prisinzano, T. E. (2013). Palladium-Catalyzed Transformations of Salvinorin A, a Neoclerodane Diterpene from Salvia divinorum. Org Lett, 15(23), 5936-5939. doi: 10.1021/ol4027528

Riley, A. P., Groer, C. E., Young, D., Ewald, A. W., Kivell, B. M., \& Prisinzano, T. E. (2014). Synthesis and kappa-Opioid Receptor Activity of Furan-Substituted Salvinorin A Analogues. Journal of Medicinal Chemistry, 57(24), 10464-10475. doi: 10.1021/jm501521d

Rinaldi, R. C., Steindler, E. M., Wilford, B. B., \& Goodwin, D. (1988). Clarification and standardization of substance abuse terminology. JAMA, 259(4), 555-557. doi: 10.1001/jama.1988.03720040047025

Ritz, M., Lamb, R., Goldberg, \& Kuhar, M. (1987). Cocaine receptors on dopamine transporters are related to self-administration of cocaine. Science, 237(4819), 1219-1223. doi: 10.1126/science. 2820058 
Roberts, D. S. (1993). Self-administration of GBR 12909 on a fixed ratio and progressive ratio schedule in rats. Psychopharmacology, 111(2), 202-206. doi: 10.1007/BF02245524

Robinson, T. E., \& Berridge, K. C. (1993). The neural basis of drug craving: An incentivesensitization theory of addiction. Brain Res Rev, 18(3), 247-291. doi: http://dx.doi.org/10.1016/0165-0173(93)90013-P

Rossi, N. A., \& Reid, L. D. (1976). Affective states associated with morphine injections. Physiological Psychology, 4(3), 269-274. doi: 10.3758/BF03332869

Roth, B. L., Baner, K., Westkaemper, R., Siebert, D., Rice, K. C., Steinberg, S., . . Rothman, R. B. (2002). Salvinorin A: A potent naturally occurring nonnitrogenous $\mathrm{k}$ opioid selective agonist. Proceedings of the National Academy of Sciences, 99(18), 11934-11939. doi: 10.1073/pnas.182234399

Russell, W. M. S., \& Burch, R. L. (1959). The principles of humane experimental technique: Methuen and Co., Ltd., London W.C.I.

Schenk, S., Lacelle, G., Gorman, K., \& Amit, Z. (1987). Cocaine self-administration in rats influenced by environmental conditions: implications for the etiology of drug abuse. Neuroscience Letters, 81(1-2), 227-231. doi: http://dx.doi.org/10.1016/03043940(87)91003-2

Schenk, S., Partridge, B., \& Shippenberg, T. S. (1999). U69593, a kappa-opioid agonist, decreases cocaine self-administration and decreases cocaine-produced drug-seeking. Psychopharmacology, 144(4), 339-346. doi: DOI 10.1007/s002130051016

Schwartz, J. W., Blakely, R. D., \& DeFelice, L. J. (2003). Binding and Transport in Norepinephrine Transporters: REAL-TIME, SPATIALLY RESOLVED ANALYSIS IN SINGLE CELLS USING A FLUORESCENT SUBSTRATE. Journal of Biological Chemistry, 278(11), 9768-9777. doi: 10.1074/jbc.M209824200

Seeman, P., Guan, H.-C., \& Hirbec, H. (2009). Dopamine D2High receptors stimulated by phencyclidines, lysergic acid diethylamide, salvinorin A, and modafinil. Synapse, 63(8), 698-704. doi: 10.1002/syn.20647

Self, D. W., Barnhart, W. J., Lehman, D. A., \& Nestler, E. J. (1996). Opposite modulation of cocaine-seeking behavior by D-1- and D-2-like dopamine receptor agonists. Science, 271(5255), 1586-1589. doi: 10.1126/science.271.5255.1586

Serra, V., Fattore, L., Scherma, M., Collu, R., Spano, M., Fratta, W., \& Fadda, P. (2015). Behavioural and neurochemical assessment of salvinorin $A$ abuse potential in the rat. Psychopharmacology, 232(1), 91-100. doi: 10.1007/s00213-014-3641-z

Shaham, Y., \& Miczek, K. (2003). Reinstatement-toward a model of relapse. Psychopharmacology, 168(1-2), 1-2. doi: 10.1007/s00213-003-1469-z

Shaw, G., Morse, S., Ararat, M., \& Graham, F. L. (2002). Preferential transformation of human neuronal cells by human adenoviruses and the origin of HEK 293 cells. The FASEB Journal, 16(8), 869-871. doi: 10.1096/fj.01-0995fje

Shippenberg, T. S., Zapata, A., \& Chefer, V. I. (2007). Dynorphin and the pathophysiology of drug addiction. Pharmacol Ther, 116(2), 306-321. doi: 10.1016/j.pharmthera.2007.06.011

Simon, H., Stinus, L., Tassin, J. P., Lavielle, S., Blanc, G., Thierry, A.-M., . . Le Moal, M. (1979). Is the dopaminergic mesocorticolimbic system necessary for intracranial self-stimulation?: Biochemical and behavioral studies from A10 cell bodies and terminals. Behavioral and Neural Biology, 27(2), 125-145. doi: http://dx.doi.org/10.1016/S0163-1047(79)91745-X

Simonin, F., Gavériaux-Ruff, C., Befort, K., Matthes, H., Lannes, B., Micheletti, G., . . Kieffer, B. (1995). kappa-Opioid receptor in humans: cDNA and genomic cloning, chromosomal assignment, functional expression, pharmacology, and expression pattern in the central nervous system. Proceedings of the National Academy of Sciences, 92(15), 7006-7010.

Simonson, B., Morani, A. S., Ewald, A. W. M., Walker, L., Kumar, N., Simpson, D., . . Kivell, B. M. (2014). Pharmacology and anti-addiction effects of the novel k opioid receptor agonist 
Mesyl Sal B, a potent and long-acting analogue of salvinorin A. British Journal of Pharmacology, n/a-n/a. doi: 10.1111/bph.12692

Simonson, B., Morani, A. S., Ewald, A. W. M., Walker, L., Kumar, N., Simpson, D., .. Kivell, B. M. (2015). Pharmacology and anti-addiction effects of the novel $k$ opioid receptor agonist Mesyl Sal B, a potent and long-acting analogue of salvinorin A. British Journal of Pharmacology, 172(2), 515-531. doi: 10.1111/bph.12692

Simpson, D. S., Katavic, P. L., Lozama, A., Harding, W. W., Parrish, D., Deschamps, J. R., . . Prisinzano, T. E. (2007). Synthetic Studies of Neoclerodane Diterpenes from Salvia divinorum: Preparation and Opioid Receptor Activity of Salvinicin Analogues. Journal of Medicinal Chemistry, 50(15), 3596-3603. doi: 10.1021/jm070393d

Siuda, Edward R., Copits, Bryan A., Schmidt, Martin J., Baird, Madison A., Al-Hasani, R., Planer, William J., . . . Bruchas, Michael R. (2015). Spatiotemporal Control of Opioid Signaling and Behavior. Neuron, 86(4), 923-935. doi: 10.1016/j.neuron.2015.03.066

Slattery, D. A., \& Cryan, J. F. (2012). Using the rat forced swim test to assess antidepressant-like activity in rodents. Nature Protocols, 7(6), 1009-1014. doi: http://dx.doi.org/10.1038/nprot.2012.044

Solomon, R. L., \& Corbit, J. D. (1974). An opponent-process theory of motivation. I. Temporal dynamics of affect. Psychological review, 81(2), 119-145.

Spanagel, R., Herz, A., \& Shippenberg, T. S. (1992). Opposing tonically active endogenous opioid systems modulate the mesolimbic dopaminergic pathway. Proceedings of the National Academy of Sciences of the United States of America, 89(6), 2046-2050. doi: 10.1073/pnas.89.6.2046

Stafford, D., LeSage, M. G., \& Glowa, J. R. (1998). Progressive-ratio schedules of drug delivery in the analysis of drug self-administration: a review. Psychopharmacology, 139(3), 169-184. doi: $10.1007 / \mathrm{s} 002130050702$

Steru, L., Chermat, R., Thierry, B., \& Simon, P. (1985). The tail suspension test: A new method for screening antidepressants in mice. Psychopharmacology, 85(3), 367-370. doi: 10.1007/BF00428203

Svingos, A. L., Chavkin, C., Colago, E. E. O., \& Pickel, V. M. (2001). Major coexpression of k-opioid receptors and the dopamine transporter in nucleus accumbens axonal profiles. Synapse, 42(3), 185-192. doi: 10.1002/syn.10005

Swanson, L. W. (1982). The projections of the ventral tegmental area and adjacent regions: A combined fluorescent retrograde tracer and immunofluorescence study in the rat. Brain Research Bulletin, 9(1-6), 321-353. doi: http://dx.doi.org/10.1016/0361-9230(82)90145$\underline{9}$

Taussig, R., Iniguez-Lluhi, J., \& Gilman, A. (1993). Inhibition of adenylyl cyclase by Gi alpha. Science, 261(5118), 218-221. doi: 10.1126/science.8327893

Taylor, D., \& Ho, B. T. (1977). Neurochemical effects of cocaine following acute and repeated injection. Journal of Neuroscience Research, 3(2), 95-101. doi: 10.1002/jnr.490030203

Teksin, Z. S., Lee, I. J., Nemieboka, N. N., Othman, A. A., Upreti, V. V., Hassan, H. E., . . Eddington, N. D. (2009). Evaluation of the transport, in vitro metabolism and pharmacokinetics of Salvinorin A, a potent hallucinogen. European Journal of Pharmaceutics and Biopharmaceutics, 72(2), 471-477. doi:

http://dx.doi.org/10.1016/i.ejpb.2009.01.002

Tempel, A., \& Zukin, R. S. (1987). Neuroanatomical patterns of the mu, delta, and kappa opioid receptors of rat brain as determined by quantitative in vitro autoradiography. Proceedings of the National Academy of Sciences, 84(12), 4308-4312.

Thomas, P., \& Smart, T. G. (2005). HEK293 cell line: A vehicle for the expression of recombinant proteins. Journal of Pharmacological and Toxicological Methods, 51(3), 187-200. doi: http://dx.doi.org/10.1016/j.vascn.2004.08.014 
Thompson, A. C., Zapata, A., Justice, J. B., Vaughan, R. A., Sharpe, L. G., \& Shippenberg, T. S. (2000). K-Opioid Receptor Activation Modifies Dopamine Uptake in the Nucleus Accumbens and Opposes the Effects of Cocaine. The Journal of Neuroscience, 20(24), 9333-9340.

Tzaferis, J. A., \& McGinty, J. F. (2001). Kappa opioid receptor stimulation decreases amphetamine-induced behavior and neuropeptide mRNA expression in the striatum. Molecular Brain Research, 93(1), 27-35. doi: http://dx.doi.org/10.1016/S0169$\underline{328 \times(01) 00178-4}$

Tzschentke, T. M. (1998). Measuring reward with the conditioned place preference paradigm: a comprehensive review of drug effects, recent progress and new issues. Progress in Neurobiology, 56(6), 613-672. doi: http://dx.doi.org/10.1016/S0301-0082(98)00060-4

UNODC. (2015). World Drug Report 2015. Vienna, Austria: United Nations Office on Drugs and Crime.

Unterwald, E. M., Rubenfeld, J. M., \& Kreek, M. J. (1994). Repeated cocaine administration upregulates kappa and mu, but not delta, opioid receptors. Neuroreport, 5(13), 16131616. doi: $10.1097 / 00001756-199408150-00018$

Ushijima, I., Carino, M. A., \& Horita, A. (1995). Involvement of D1 and D2 dopamine systems in the behavioral effects of cocaine in rats. Pharmacology Biochemistry and Behavior, 52(4), 737-741. doi: http://dx.doi.org/10.1016/0091-3057(95)00167-U

Valdes, L. J. (1983). The Pharmacognosy of Salvia divinorum (Epling and Jativa-M): an investigation of Ska Maria Pastora (Mexico). (8402393 Ph.D.), University of Michigan, Ann Arbor. ProQuest Dissertations \& Theses Global database.

Valdez, G. R., Platt, D. M., Rowlett, J. K., Rüedi-Bettschen, D., \& Spealman, R. D. (2007). k Agonist-Induced Reinstatement of Cocaine Seeking in Squirrel Monkeys: A Role for Opioid and Stress-Related Mechanisms. Journal of Pharmacology and Experimental Therapeutics, 323(2), 525-533. doi: 10.1124/jpet.107.125484

Vanderschuren, L. J. M. J., Schoffelmeer, A. N. M., Wardeh, G., \& De Vries, T. J. (2000). Dissociable effects of the $\mathrm{k}$-opioid receptor agonists bremazocine, U69593, and $\mathrm{U} 50488 \mathrm{H}$ on locomotor activity and long-term behavioral sensitization induced by amphetamine and cocaine. Psychopharmacology, 150(1), 35-44. doi: $10.1007 / \mathrm{s} 002130000424$

Vargas-Perez, H., Ting-A-Kee, R. A., Heinmiller, A., Sturgess, J. E., \& Van Der Kooy, D. (2007). A test of the opponent-process theory of motivation using lesions that selectively block morphine reward. European Journal of Neuroscience, 25(12), 3713-3718. doi: 10.1111/j.1460-9568.2007.05599.x

Volkow, N. D., Chang, L., Wang, G.-J., Fowler, J. S., Franceschi, D., Sedler, M., . . Logan, J. (2001). Loss of Dopamine Transporters in Methamphetamine Abusers Recovers with Protracted Abstinence. The Journal of Neuroscience, 21(23), 9414-9418.

Volkow, N. D., Fowler, J. S., Wang, G.-J., Hitzemann, R., Logan, J., Schlyer, D. J., . . Wolf, A. P. (1993). Decreased dopamine D2 receptor availability is associated with reduced frontal metabolism in cocaine abusers. Synapse, 14(2), 169-177. doi: 10.1002/syn.890140210

Volkow, N. D., Wang, G. J., Fischman, M. W., Foltin, R. W., Fowler, J. S., Abumrad, N. N., . . Shea, C. E. (1997). Relationship between subjective effects of cocaine and dopamine transporter occupancy. Nature, 386(6627), 827-830.

Volkow, N. D., Wang, G. J., Fowler, J. S., Logan, J., Gatley, S. J., Hitzemann, R., . . Pappas, N. (1997). Decreased striatal dopaminergic responsiveness in detoxified cocaine-dependent subjects. Nature, 386(6627), 830-833.

Walker, B. M., Valdez, G. R., McLaughlin, J. P., \& Bakalkin, G. (2012). Targeting dynorphin/kappa opioid receptor systems to treat alcohol abuse and dependence. Alcohol, 46(4), 359-370. doi: http://dx.doi.org/10.1016/j.alcohol.2011.10.006 
Walker, B. M., Zorrilla, E. P., \& Koob, G. F. (2011). Systemic k-opioid receptor antagonism by norbinaltorphimine reduces dependence-induced excessive alcohol self-administration in rats. Addiction Biology, 16(1), 116-119. doi: 10.1111/j.1369-1600.2010.00226.x

Walsh, S., Strain, E., Abreu, M., \& Bigelow, G. (2001). Enadoline, a selective kappa opioid agonist: comparison with butorphanol and hydromorphone in humans. Psychopharmacology, 157(2), 151-162. doi: 10.1007/s002130100788

Wang, Y., Tang, K., Inan, S., Siebert, D., Holzgrabe, U., Lee, D. Y. W., ... Liu-Chen, L. Y. (2005). Comparison of pharmacological activities of three distinct kappa ligands (salvinorin $A$, TRK-820 and 3FLB) on kappa opioid receptors in vitro and their antipruritic and antinociceptive activities in vivo. Journal of Pharmacology and Experimental Therapeutics, 312(1), 220-230. doi: 10.1124/jpet.104.073668

Wang, Y. H., Chen, Y., Xu, W., Lee, D. Y. W., Ma, Z., Rawls, S. M., . . Liu-Chen, L.-Y. (2008). 2Methoxymethyl-Salvinorin B Is a Potent k Opioid Receptor Agonist with Longer Lasting Action in Vivo Than Salvinorin A. Journal of Pharmacology and Experimental Therapeutics, 324(3), 1073-1083. doi: 10.1124/jpet.107.132142

Wee, S., \& Koob, G. F. (2010). The role of the dynorphin-kappa opioid system in the reinforcing effects of drugs of abuse. Psychopharmacology, 210(2), 121-135. doi: 10.1007/s00213010-1825-8

Wee, S., Orio, L., Ghirmai, S., Cashman, J. R., \& Koob, G. F. (2009). Inhibition of kappa opioid receptors attenuated increased cocaine intake in rats with extended access to cocaine. Psychopharmacology, 205(4), 565-575. doi: 10.1007/s00213-009-1563-y

Weeks, J. R. (1962). Experimental Morphine Addiction: Method for Automatic Intravenous Injections in Unrestrained Rats. Science, 138(3537), 143-144. doi: 10.1126/science.138.3537.143

Whitfield, T. W., Schlosburg, J. E., Wee, S., Gould, A., George, O., Grant, Y., ... Koob, G. F. (2015). $\kappa$ Opioid Receptors in the Nucleus Accumbens Shell Mediate Escalation of Methamphetamine Intake. The Journal of Neuroscience, 35(10), 4296-4305. doi: 10.1523/jneurosci.1978-13.2015

WHO. (2010). Psychoactive Substance Use: Epidemiology and Burden of Disease. Atlas on Substance Use. Geneva: WHO Press, 2010; 7-12.

Wickizer, T. M. (2013). State-level estimates of the economic costs of alcohol and drug abuse. Journal of health care finance, 39(3), 71-84.

Wilkins, C., Girling, M., \& Sweetsur, P. (2008). Recent trends in illegal drug use in New Zealand, 2005-2007: Findings from the 2005, 2006 and 2007 Illicit Drug Monitoring System (IDMS). Auckland, New Zealand: Centre for Social and Health Outcones Research and Evaluation, Massey University.

Wilkins, C., Girling, M., \& Sweetsur, P. (2008). Trends in drug use in Auckland, Wellington and Christchurch: findings from the three site locations of the 2006 and 2007 Illicit Drug Monitoring System (IDMS). Auckland, New Zealand: Centre for Social and Health Outcomes Research and Evaluation, Massey University.

Willner, P., Papp, M., Phillips, G., Maleeh, M., \& Muscat, R. (1990). Pimozide does not impair sweetness discrimination. Psychopharmacology, 102(2), 278-282. doi: 10.1007/BF02245934

Wood, P. L., \& Rao, T. S. (1991). Morphine stimulation of mesolimbic and mesocortical but not nigrostriatal dopamine release in the rat as reflected by changes in 3-methoxytyramine levels. Neuropharmacology, 30(4), 399-401. doi: 10.1016/0028-3908(91)90066-k

Wrighton, S. A., \& Stevens, J. C. (1992). The Human Hepatic Cytochromes P450 Involved in Drug Metabolism. Critical Reviews in Toxicology, 22(1), 1-21. doi: 10.3109/10408449209145319

Wu, H., Wacker, D., Mileni, M., Katritch, V., Han, G. W., Vardy, E., ... Stevens, R. C. (2012). Structure of the human kappa-opioid receptor in complex with JDTic. Nature, 485(7398), 
327-332. doi:

http://www.nature.com/nature/journal/v485/n7398/abs/nature10939.html\#supplemen tary-information

Xia, Y.-F., He, L., Whistler, J. L., \& Hjelmstad, G. O. (2008). Acute Amphetamine Exposure Selectively Desensitizes Kappa-Opioid Receptors in the Nucleus Accumbens. Neuropsychopharmacology, 33(4), 892-900. doi: http://dx.doi.org/10.1038/sj.npp.1301463

Xu, M., Hu, X.-T., Cooper, D. C., Moratalla, R., Graybiel, A. M., White, F. J., \& Tonegawa, S. (1994). Elimination of cocaine-induced hyperactivity and dopamine-mediated neurophysiological effects in dopamine D1 receptor mutant mice. Cell, 79(6), 945-955. doi: http://dx.doi.org/10.1016/0092-8674(94)90026-4

Yeo, K. T., Wu, A. H., Apple, F. S., Kroll, M. H., Christenson, R. H., Lewandrowski, K. B., . . Butch, A. W. (2003). Multicenter evaluation of the Roche NT-proBNP assay and comparison to the Biosite Triage BNP assay. Clin Chim Acta, 338(1-2), 107-115.

Yoshida, M., Yokoo, H., Mizoguchi, K., Kawahara, H., Tsuda, A., Nishikawa, T., \& Tanaka, M. (1992). Eating and drinking cause increased dopamine release in the nucleus accumbens and ventral tegmental area in the rat: measurement by in vivo microdialysis. Neuroscience Letters, 139(1), 73-76. doi: http://www.sciencedirect.com/science/article/pii/030439409290861Z

Zakharova, E., Collins, S. L., Åberg, M., Kumar, A., Fernandez, J. B., \& Izenwasser, S. (2008). Depletion of serotonin decreases the effects of the kappa-opioid receptor agonist U69593 on cocaine-stimulated activity. Eur J Pharmacol, 586(1-3), 123-129. doi: http://dx.doi.org/10.1016/j.ejphar.2008.02.065

Zapata, A., Kivell, B., Han, Y., Javitch, J. A., Bolan, E. A., Kuraguntla, D., ... Shippenberg, T. S. (2007). Regulation of dopamine transporter function and cell surface expression by D3 dopamine receptors. Journal of Biological Chemistry, 282(49), 35842-35854. doi: DOI 10.1074/jbc.M611758200

Zhang, Y., Butelman, E. R., Schlussman, S. D., Ho, A., \& Kreek, M. J. (2005). Effects of the plantderived hallucinogen salvinorin $A$ on basal dopamine levels in the caudate putamen and in a conditioned place aversion assay in mice: agonist actions at kappa opioid receptors. Psychopharmacology, 179(3), 551-558. doi: 10.1007/s00213-004-2087-0

Zhou, L., Lovell, K. M., Frankowski, K. J., Slauson, S. R., Phillips, A. M., Streicher, J. M., . . Bohn, L. M. (2013). Development of Functionally Selective, Small Molecule Agonists at Kappa Opioid Receptors. Journal of Biological Chemistry, 288(51), 36703-36716. doi: 10.1074/jbc.M113.504381

Zhu, N., Lightsey, D., Liu, J., Foroozesh, M., Morgan, K., Stevens, E., \& Stevens, C. K. (2010). Ethynyl and Propynylpyrene Inhibitors of Cytochrome P450. Journal of Chemical Crystallography, 40(4), 343-352. doi: 10.1007/s10870-009-9659-0 
